\title{
Magnetosphere-ionosphere coupling currents in Jupiter's middle magnetosphere: effect of precipitation-induced enhancement of the ionospheric Pedersen conductivity
}

\author{
J. D. Nichols and S. W. H. Cowley \\ Department of Physics and Astronomy, University of Leicester, Leicester LE1 7RH, UK \\ Received: 3 October 2003 - Revised: 8 January 2004 - Accepted: 23 January 2004 - Published: 8 April 2004
}

\begin{abstract}
We consider the effect of precipitation-induced enhancement of the Jovian ionospheric Pedersen conductivity on the magnetosphere-ionosphere coupling current system which is associated with the breakdown of the corotation of iogenic plasma in Jupiter's middle magnetosphere. In previous studies the Pedersen conductivity has been taken to be simply a constant, while it is expected to be significantly enhanced in the regions of upward-directed auroral field-aligned current, implying downward precipitating electrons. We develop an empirical model of the modulation of the Pedersen conductivity with field-aligned current density based on the modelling results of Millward et al. and compute the currents flowing in the system with the conductivity self-consistently dependent on the auroral precipitation. In addition, we consider two simplified models of the conductivity which provide an insight into the behaviour of the solutions. We compare the results to those obtained when the conductivity is taken to be constant, and find that the empirical conductivity model helps resolve some outstanding discrepancies between theory and observation of the plasma angular velocity and current system. Specifically, we find that the field-aligned current is concentrated in a peak of magnitude $\sim 0.25 \mu \mathrm{A} \mathrm{m}^{-2}$ in the inner region of the middle magnetosphere at $\sim 20 R_{J}$, rather than being more uniformly distributed as found with constant conductivity models. This peak maps to $\sim 17^{\circ}$ in the ionosphere, and is consistent with the position of the main oval auroras. The energy flux associated with the field-aligned current is $\sim 10 \mathrm{~mW} \mathrm{~m}^{-2}$ (corresponding to a UV luminosity of $\sim 100 \mathrm{kR}$ ), in a region $\sim 0.6^{\circ}$ in width, and the Pedersen conductivity is elevated from a background of $\sim 0.05 \mathrm{mho}$ to $\sim 0.7 \mathrm{mho}$. Correspondingly, the total equatorial radial current increases greatly in the region of peak field-aligned current, and plateaus with increasing distance thereafter. This form is consistent with the observed profile of the current derived from Galileo magnetic field data. In addition, we find that the solutions using the
\end{abstract}

Correspondence to: J. D. Nichols

(jdn@ion.le.ac.uk) empirical conductivity model produce an angular velocity profile which maintains the plasma near to rigid corotation out to much further distances than the constant conductivity model would suggest. Again, this is consistent with observations. Our results therefore suggest that, while the constant conductivity solutions provide an important indication that the main oval is indeed a result of the breakdown of the corotation of iogenic plasma, they do not explain the details of the observations. In order to resolve some of these discrepancies, one must take into account the elevation of the Pedersen conductivity as a result of auroral electron precipitation.

Key words. Magnetospheric physics (current systems, magnetosphere-ionosphere interactions, planetary magnetospheres)

\section{Introduction}

Interest in the magnetosphere-ionosphere coupling currents that flow in Jupiter's middle magnetosphere has been enhanced considerably in recent years by the suggestion of a number of authors that they are directly associated with the Jovian main oval auroras (Bunce and Cowley, 2001; Hill, 2001; Khurana, 2001; Southwood and Kivelson, 2001). The implication follows that the dynamics of this region may be sensed remotely by ground- and space-based auroral observations covering a wide range of wavelengths (e.g. Satoh et al., 1996; Clarke et al., 1998; Prangé et al., 1998; Vasavada et al., 1999; Pallier and Prangé, 2001; Grodent et al., 2003). An outline of the physics of the middle magnetosphere region is shown in Fig. 1, forming a background to the analysis and results which follow. By far the most important plasma component in the middle magnetosphere originates from the atmosphere of the moon Io, which orbits deep within the magnetospheric cavity at an equatorial radial distance of $\sim 6 R_{J}$ (Hill et al., 1983; Vasyliunas, 1983). ( $R_{J}$ is Jupiter's radius, taken here to be equal to $71323 \mathrm{~km}$.) Sulphur and oxygen ions and electrons that are produced from the neutral gas clouds that 


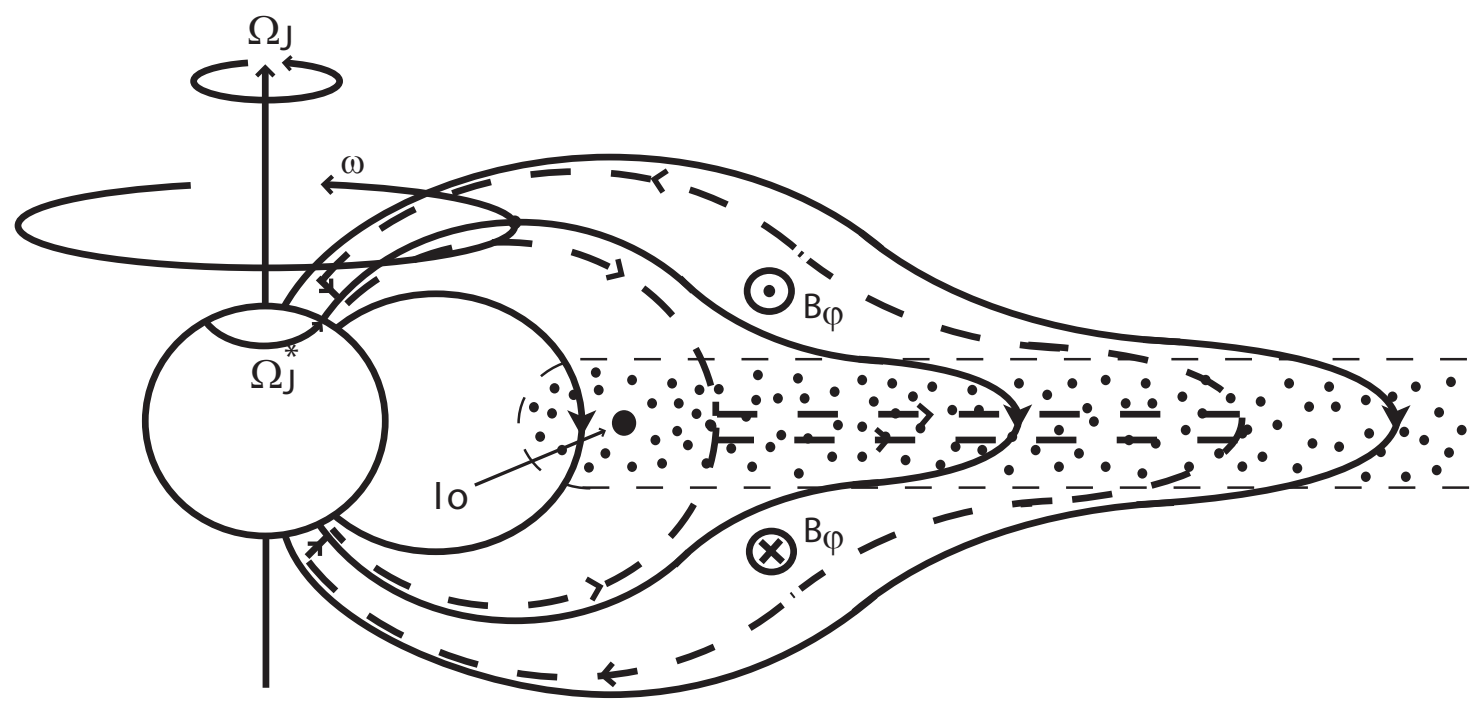

Fig. 1. Sketch of a meridian cross section through Jupiter's inner and middle magnetosphere, showing the principal physical features involved. The arrowed solid lines indicate magnetic field lines, the arrowed dashed lines the magnetosphere-ionosphere coupling current system, and the dotted region the rotating disc of out-flowing iogenic plasma (from Cowley and Bunce, 2001).

co-orbit with Io are picked up by the plasma flow, forming a dense near-corotating plasma torus in the vicinity of Io's orbit (e.g. Belcher, 1983; Bagenal, 1994). A significant fraction of the plasma ions that are produced in the torus undergo charge-exchange reactions with the neutral gas, and escape from the system as fast neutral particles (e.g. Pontius and Hill, 1982). The remainder flow outward under the action of the centrifugal force through flux tube interchange motions, to form a vast spinning equatorial plasma disc, shown dotted in Fig. 1 (e.g. Siscoe and Summers, 1981). Estimates of the total plasma production rate within the torus lie typically within the range of $1000-3000 \mathrm{~kg} \mathrm{~s}^{-1}$ when both the local production near Io and the more distributed production in the co-orbiting gas clouds are included (e.g. Broadfoot et al., 1981; Brown, 1994; Bagenal, 1997; Delamere and Bagenal, 2003). Of this total, perhaps one-third to one-half emerges into the plasma outflow in the equatorial plasma disc. Khurana and Kivelson (1993) derived a lower limit on the outward mass transport rate through the disc of $\sim 500 \mathrm{~kg} \mathrm{~s}^{-1}$ from consideration of the magnetic torque exerted on the plasma, as first discussed by Vasyliunas (1983). In addition, Hill (1980) estimated an outward mass transport rate of $\sim 2000 \mathrm{~kg} \mathrm{~s}^{-1}$ based on Voyager angular velocity profiles, though this estimate also requires knowledge of the heightintegrated Jovian ionospheric Pedersen conductivity, taken to be $\sim 0.05$ mho. Overall, however, it seems reasonable to suppose that the outward transport rate of plasma through the disc is typically $\sim 1000 \mathrm{~kg} \mathrm{~s}^{-1}$, with variations of perhaps a factor of $\sim 2$ on either side. The flux tubes threading this disc constitute the Jovian middle magnetosphere, which stretches typically over many tens of $R_{J}$ from the vicinity of Io's orbit to the vicinity of the magnetopause, depending on local time and the degree of extension of the magnetosphere determined by solar wind conditions. The field lines in this region are characteristically distended radially outward from the planet, as shown in the figure, due to azimuthal currents flowing in the equatorial plasma associated with radial stress balance. The iogenic plasma is eventually lost from the outer regions of the disc by processes which are at present not well determined, but may involve plasmoid formation and outflow in the dusk sector of the magnetic tail (Vasyliunas, 1983).

As the iogenic plasma diffuses outward from the torus its angular velocity will drop below near-rigid corotation with the planet, inversely as the square of the distance if no torques act. However, when the angular velocity of the plasma and frozen-in field lines ( $\omega$ in Fig. 1) falls below that of the planet $\left(\Omega_{J}\right)$, or more specifically, below that of the neutral upper atmosphere in the ionospheric Pedersen layer $\left(\Omega_{J}^{*}\right)$, ion-neutral collisions in the Pedersen layer provide a torque on the feet of the field lines which tends to spin the plasma back up towards rigid corotation. The torque is communicated to the equatorial plasma by the bending of the magnetic field lines out of meridian planes, associated with the azimuthal field components $B_{\varphi}$ shown in Fig. 1, which reverse sense across the equator. The associated electric current system, which is the principal topic of the present paper, is shown by the dashed lines in the figure. It consists of an outward-flowing radial current in the equatorial plane associated with the reversal in the azimuthal field, which is connected to equatorward-directed Pedersen currents in both hemispheres by field-aligned currents which flow outward from the ionosphere to the magnetosphere in the inner part of the system, and return from the magnetosphere to the ionosphere in the outer part (Hill, 1979; Vasyliunas, 1983). It is with the upward-directed field-aligned currents in this system, carried by downward-precipitating magnetospheric electrons, that the main oval auroras have been suggested to be associated. The torque associated with the $\boldsymbol{j} \times \boldsymbol{B}$ force 
of the Pedersen current balances the frictional torque on the ionospheric plasma due to ion-neutral collisions, while the equal and opposite torque on the equatorial plasma tends to spin the plasma up towards rigid corotation with the planet. In the steady state, the plasma angular velocity will still generally fall with increasing distance, but at a rate which is less than the inverse square dependence expected from conservation of angular momentum.

Hill (1979) was the first to calculate the steady-state plasma angular velocity profile using Newton's second law, assuming a dipole poloidal magnetic field, a constant plasma mass outflow rate from the torus $\dot{M}$, and a constant value of the ionospheric Pedersen conductivity $\Sigma_{P}$. Later, Huang and Hill (1989) pointed out that the corresponding frictional torque on the neutral atmosphere in the Pedersen layer would result in the latter sub-corotating relative to the planet at an angular velocity $\Omega_{J}^{*}$, which is intermediate between that of the planet $\Omega_{J}$ and that of the plasma $\omega$. This lowers the atmospheric torque at the feet of the field lines for a given sub-corotational plasma angular velocity, an effect parameterised in the theory via use of an "effective" value of the ionospheric Pedersen conductivity $\Sigma_{P}^{*}$, which is less than the true value $\Sigma_{P}$. Subsequently, Pontius (1997) extended Hill's (1979) calculations to include a realistic empiricallybased poloidal magnetic field model, but found that the solutions for the steady-state equatorial angular velocity are remarkably insensitive to the model employed. In neither of these studies were the properties of the associated electric current system explicitly calculated. However, with the realisation of the probable connection with the main oval auroras, this omission was corrected by Hill (2001) for the case of the original model based on a dipole poloidal field, and by Cowley and Bunce (2001) for both a dipole field and for a realistic middle magnetosphere current sheet field. The latter study showed that the upward-directed field-aligned current density for the current sheet field model is typically more than an order of magnitude larger than that obtained for the dipole, and is sufficiently intense to require downward-acceleration of magnetospheric electrons through field-aligned voltages of several tens of $\mathrm{kV}$, such that the precipitating electron energy flux will produce main oval auroras exceeding $\sim 100 \mathrm{kR}$ in intensity, as observed. The origin of the field-aligned current enhancement effect lies in the differing mapping of field lines between the equatorial plane and the ionosphere in the two models, as discussed further by Cowley et al. (2002, 2003). Most recently, Nichols and Cowley (2003) have explored steady-state solutions for the plasma angular velocity and current system over wide ranges of the system parameters $\dot{M}$ and $\Sigma_{P}^{*}$, and have confirmed the general validity of these conclusions.

An important limitation of these calculations, however, is that they all assume a constant value of the effective ionospheric Pedersen conductivity, though Pontius (1997) discussed the effect of variations of this parameter produced by varying atmospheric "slippage" associated with the possible onset of instabilities induced by atmospheric velocity shear. However, given the newly-understood linkage with the main oval auroras, it seems clear that a far more important conductivity modulation mechanism results from the ionospheric ionisation produced by precipitating accelerated main oval electrons. For example, Strobel and Atreya (1983) estimated that a Pedersen conductivity of a fraction of an mho produced solely by solar UV radiation could be increased to $\sim 10$ mho (uncorrected for atmospheric slippage) under conditions of intense auroral precipitation. These conclusions have recently been confirmed by the more detailed calculations of Millward et al. (2002), using the JIM Jovian coupled ionosphere-thermosphere model of Achilleos et al. (1998). Millward et al. (2002) calculated the effect on the ionospheric conductivity of precipitating monoenergetic electron beams, and found, for example, that a beam of $\sim 60 \mathrm{keV}$ electrons (comparable to the energies determined by Cowley and Bunce, 2001) associated with an upward field-aligned current of $\sim 1 \mu \mathrm{A} \mathrm{m}^{-2}$ (on the high side of those determined here) will produce an increase in height-integrated Pedersen conductivity from a background value of less than 0.1 mho to a value of $\sim 7.5$ mho (uncorrected for atmospheric slippage).

It is the primary purpose of the present paper to investigate the effect on the plasma angular velocity profiles, and on the magnetosphere-ionosphere current circuit, of precipitationinduced changes in the ionospheric conductivity. In so doing, we will also address two other issues left open from previous studies. The first is the issue addressed specifically by Pontius (1997), which concerns the fact that observed values of the plasma angular velocity in the outer part of the middle magnetosphere (e.g. Kane et al., 1995; Krupp et al., 2001) do not fall as fast with increasing radial distance as anticipated from simple constant-conductivity models. Cowley and Bunce (2001) anticipated that such effects could result from precipitation-induced enhancements in the ionospheric conductivity and the consequent atmospheric torque. The second concerns the radial profile of the outward-directed field-aligned currents which feed the radial current in the equatorial plasma. In the constant-conductivity models derived to date, these currents are broadly distributed over the whole middle magnetosphere region, such that the equatorial radial current, integrated over local time, grows monotonically with increasing distance (see, e.g. Nichols and Cowley, 2003), closing in the exterior region outside the middle magnetosphere not described by the model. However, analysis of Galileo magnetometer data presented by Khurana (2001) indicates that the outward field-aligned current into the equatorial plane is actually concentrated in the inner region between radial distances of $\sim 10$ and $\sim 30 R_{J}$, peaking near $20 R_{J}$, such that the total radial current, integrated in local time, is approximately constant at distances beyond. These results for the location of the upward field-aligned current are also in rough accord with the observed locations of the main oval auroras (Clarke et al. 1998; Prangé et al., 1998; Grodent et al., 2003). Here we will show that the effect of precipitationinduced enhancement of the ionospheric conductivity is just such as to concentrate the upward current into the inner middle magnetosphere region, as deduced from the Galileo data. 
This paper is organised as follows. In the next section the basic theoretical framework will be outlined for the poloidal field model and the coupling current system, the latter allowing for the inclusion of a varying height-integrated ionospheric Pedersen conductivity. In Sect. 3 we then derive an empirical model of how the conductivity depends on the field-aligned current, estimated from the results presented by Millward et al. (2002). In Sect. 4 we present solutions for the plasma angular velocity and the current using simplified conductivity models motivated by the empirical results, allowing us to investigate the nature of the solutions and their relation to the previous solutions derived for constant conductivity. In Sect. 5 we then present results using the full empirical conductivity model, and compare them with the current profiles derived by Khurana (2001) from Galileo data. Our results are finally summarised in Sect. 6.

\section{Basic theory}

In this section we summarise the theoretical basis for the calculations presented in this paper. It is essentially similar to that discussed previously by Hill (1979, 2001), Pontius (1997), Cowley and Bunce (2001), and Cowley et al. (2002, 2003), except that here the ionospheric Pedersen conductivity is treated as a varying quantity rather than simply as a constant. Consequently, we only present an outline of the central results, together with the approximations and assumptions that have been made.

\subsection{Magnetic field model}

The magnetic field in our model is assumed to be axisymmetric, as is appropriate to the nature of Jupiter's middle magnetosphere. In this case the poloidal field can be specified by a flux function, $F(\rho, z)$ which is related to the field components by $\boldsymbol{B}=(1 / \rho) \nabla F \times \hat{\varphi}$, where $\rho$ is the perpendicular distance from the magnetic axis, $z$ is the distance along this axis from the magnetic equator, and $\varphi$ is the azimuthal angle. In this case $F=$ constant defines a flux shell, such that magnetic mapping between the equatorial plane and the ionosphere is achieved simply by writing $F_{e}=F_{i}$. Neglecting non-dipole planetary fields and small perturbations due to magnetospheric current systems, the flux function in the ionosphere is taken to be

$F_{i}=B_{J} \rho_{i}^{2}=B_{J} R_{J}^{2} \sin ^{2} \theta_{i}$

where $\rho_{i}$ is the perpendicular distance from the magnetic axis, $\theta_{i}$ is the magnetic co-latitude, and $B_{J}$ the dipole equatorial magnetic field strength (taken to be $426400 \mathrm{nT}$ in conformity with the VIP 4 internal field model of Connerney et al., 1998). The absolute value of $F$ has been fixed by taking $F=0$ on the magnetic axis.

The flux function in the equatorial plane can be obtained by integrating

$B_{z e}=\frac{1}{\rho_{e}} \frac{d F_{e}}{d \rho_{e}}$, where $B_{z e}$ is the north-south magnetic field which threads through the equatorial plane. In previous modelling work we have used the "Voyager-1/Pioneer-10" model of Connerney et al. (1981) (the "CAN" model) at small distances, and the Voyager-1 model of Khurana and Kivelson (1993) (the "KK" model) at large distances, the models being joined at an equatorial radial distance of $\rho_{e}^{*} \approx 21.78 R_{J}$, where the two model curves meet (Cowley and Bunce, 2001; Cowley et al., 2002, 2003). Although there is then no discontinuity in the field magnitude at this point, there is a discontinuity in the first derivative. Here instead we employ a field model which is very close to that previously used, but is continuous over the range of interest. The model equatorial field is given by

$$
\begin{aligned}
& B_{z e}\left(\rho_{e}\right)= \\
& \quad-\left\{B_{o}\left(\frac{R_{J}}{\rho_{e}}\right)^{3} \exp \left[-\left(\frac{\rho_{e}}{\rho_{e o}}\right)^{5 / 2}\right]+A\left(\frac{R_{J}}{\rho_{e}}\right)^{m}\right\},
\end{aligned}
$$

where $B_{o}=3.335 \times 10^{5} \mathrm{nT}, \rho_{e o}=14.501 R_{J}, A=5.4 \times 10^{4} \mathrm{nT}$ and $m=2.71$. The second term in this expression is simply the KK model, applicable at large distances, while the first term is a modified dipole in form. The corresponding flux function is given by

$$
\begin{aligned}
& F_{e}\left(\rho_{e}\right)=F_{\infty} \\
& \quad+\frac{B_{o} R_{J}^{3}}{2.5 \rho_{e o}} \Gamma\left[-\frac{2}{5},\left(\frac{\rho_{e}}{\rho_{e o}}\right)^{5 / 2}\right]+\frac{A}{(m-2)}\left(\frac{R_{J}}{\rho_{e}}\right)^{m-2},
\end{aligned}
$$

where $F_{\infty} \approx 2.841 \times 10^{4} \mathrm{nT} R_{J}^{2}$ is the value of the flux function at infinity, and $\Gamma(a, z)$ is the incomplete gamma function $\Gamma(a, z)=\int_{z}^{\infty} t^{a-1} e^{-t} d t$. This field model is such that the values of both $B_{z e}$ and $F_{e}$ at the inner edge of the middle magnetosphere current sheet at $\rho_{e}=5 R_{J}$ are exactly those given by the CAN model (in the Edwards et al., 2001 approximations). These values are $\sim 3177 \mathrm{nT}$ and $\sim 8.819 \times 10^{4} \mathrm{nT} R_{J}^{2}$, compared with $\sim 3411 \mathrm{nT}$ and $\sim 8.528 \times 10^{4} \mathrm{nT} R_{J}^{2}$ for the dipole field alone, the differences reflecting the stretched out nature of the field due to the presence of the current sheet. The model is also such that the flux threading through the current sheet between $5 R_{J}$ and infinity is exactly equal to that of the CAN/KK model employed previously, such that the values of $F_{\infty}$ in the two models are also exactly equal. Consequently, the models map into exactly the same dipole latitude band in the ionosphere, spanning dipole co-latitudes between $\sim 27^{\circ}$ and $\sim 15^{\circ}$ for radial distances between $5 R_{J}$ and infinity. In Fig. 2 we show plots of $\left|B_{z e}\right|$ (the actual values are, of course, all negative), $F_{e}$, and the ionospheric co-latitude $\theta_{i}$ versus equatorial radial distance over the range 0 to $100 R_{J}$ (as employed throughout this paper). The solid lines show the values for the above field model, while the dashed lines show the values for the planetary dipole alone. The dotted lines in the $\left|B_{z e}\right|$ plot show the values for the $\mathrm{CAN} / \mathrm{KK}$ model, which are only clearly 
visible when they are projected beyond $\rho_{e}^{*}$. The ionospheric mapping is given from Eq. (1) by

$\sin \theta_{i}=\sqrt{\frac{F_{e}\left(\rho_{e}\right)}{B_{J} R_{J}^{2}}}$,

such that the outermost field line in the plot, mapping to $100 R_{J}$ in the equatorial plane, maps to $\sim 15.7^{\circ}$ in the ionosphere. The horizontal dashed lines in the $F_{e}$ and $\theta_{i}$ plots show the asymptotic values of these quantities at large distances.

\subsection{Current system}

We now consider the calculation of the components of the current system illustrated in Fig. 1, in terms of the profile of the plasma angular velocity as a function of flux shell, $\omega(F)$. The calculation of $\omega(F)$ from Newton's second law then follows in Sect. 2.3. First, the equatorward-directed height integrated Pedersen current is given by

$i_{P}=2 \Sigma_{P}^{*} B_{J} \rho_{i}\left(\Omega_{J}-\omega\right)$,

where, as introduced briefly above, $\Omega_{J}$ is Jupiter's angular velocity $\left(1.76 \times 10^{-4} \mathrm{rad} \mathrm{s}^{-1}\right), \Sigma_{P}^{*}$ is the effective value of the height-integrated Pedersen conductivity, and we have taken the polar ionospheric field to be vertical and equal to $2 B_{J}$ in strength. The effective value of the Pedersen conductivity is related to the true value by

$\Sigma_{P}^{*}=(1-k) \Sigma_{P}$,

where, as indicated above, parameter $k$ is related to the slippage of the neutral atmosphere from strict rigid corotation

$\left(\Omega_{J}-\Omega_{J}^{*}\right)=k\left(\Omega_{J}-\omega\right)$.

Continuity in the current circuit shown in Fig. 1 then requires that the equatorial radial current integrated across the width of the current sheet is given by

$\rho_{e} i_{\rho}=2 \rho_{i} i_{P}$

where we have assumed north-south symmetry. Introducing Eq. (5) into Eq. (8), and noting from Eq. (1) that $F_{e}=F_{i}=B_{J} \rho_{i}^{2}$ on a flux shell, then gives

$i_{\rho}=\frac{4 \Sigma_{P}^{*} F_{e}\left(\Omega_{J}-\omega\right)}{\rho_{e}}$,

such that the total radial current, integrated in azimuth, is

$I_{P}=2 \pi \rho_{e} i_{\rho}=8 \pi \Sigma_{P}^{*} F_{e}\left(\Omega_{J}-\omega\right)$,

equal, of course, to twice the azimuth-integrated Pedersen current in each conjugate ionosphere, $I_{P}$. The field-aligned current density can then be computed from the divergence of either $I_{P}$ or $I_{\rho}$. In terms of $I_{\rho}$ we find

$\left(\frac{j_{\|}}{B}\right)=\frac{1}{4 \pi \rho_{e}\left|B_{z e}\right|} \frac{d I_{\rho}}{d \rho_{e}}$,
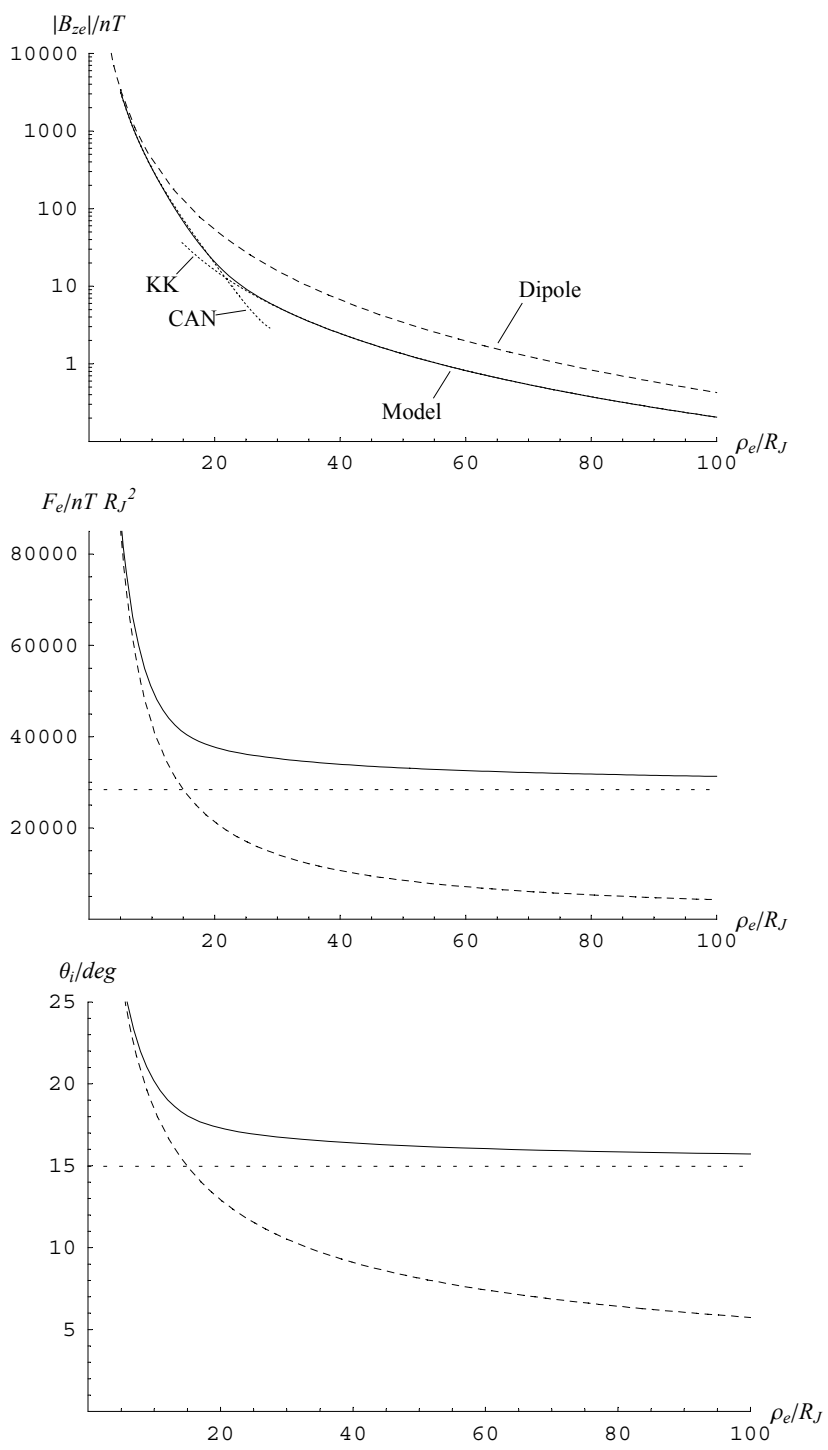

Fig. 2. Plots showing the parameters of the current sheet field model employed in this paper (solid lines) compared with values for the planetary dipole field alone (dashed lines). Plot (a) is a log-linear plot of the modulus of the north-south component of the equatorial magnetic field $\left|B_{z e}\right|$ in $\mathrm{nT}$ threading the equatorial plane, shown versus jovicentric equatorial radial distance $\rho_{e}$, and where we note that the actual values are negative (i.e. the field points south). The solid line shows the field model employed in this paper, given by Eq. (3a), which is based on the CAN-KK model of previous papers. The dotted lines show the CAN and KK models themselves, plotted beyond their intersection for ease of visibility. Plot (b) similarly shows the equatorial flux function of the model field $F_{e}$ in nT $R_{J}^{2}$ versus jovicentric equatorial radial distance $\rho_{e}$, given by Eq. (3b). The dotted line shows the value of the flux function at infinity, $F_{\infty}$. Plot (c) shows the mapping of the field lines between the equatorial plane and the ionosphere, determined from Eq. (4). The ionospheric co-latitude of the field line $\theta_{i}$ is plotted versus jovicentric equatorial radial distance $\rho_{e}$. The dotted line shows the ionospheric co-latitude of the field line which maps to infinity in the equatorial plane for our current sheet field model. 
where $\left(j_{\|} / B\right)$ is constant along field lines between the equatorial current sheet and the ionosphere in the assumed absence of significant field-perpendicular currents in this region. Hence, the field-aligned current just above the ionosphere is given by

$$
\begin{gathered}
j_{\| i}=2 B_{J}\left(\frac{j_{\|}}{B}\right)=\frac{B_{J}}{2 \pi \rho_{e}\left|B_{z e}\right|} \frac{d I_{\rho}}{d \rho_{e}}= \\
\frac{4 B_{J} \Omega_{J}}{\pi \rho_{e}\left|B_{z e}\right|} \frac{d}{d \rho_{e}}\left[\Sigma_{P}^{*} F_{e}\left(1-\frac{\omega}{\Omega_{J}}\right)\right] .
\end{gathered}
$$

We note specifically in Eq. (12) that we take the heightintegrated effective Pedersen conductivity to vary with position at the feet of the field lines, such that it must be retained within the differential.

2.3 Steady-state plasma and field line angular velocity profile

As derived previously by Hill (1979) and Pontius (1997), and as discussed further by Cowley et al. (2002), application of Newton's second law to a steady flow of plasma from the Io torus yields

$$
\frac{d}{d \rho_{e}}\left(\dot{M} \rho_{e}^{2} \omega\right)=\rho_{e} I_{\rho}\left|B_{z e}\right|,
$$

where the LHS is the radial gradient of the outward plasma angular momentum flux, and the RHS is the azimuthintegrated electromagnetic torque per unit radius. Parameter $\dot{M}$ in this equation is the plasma mass per second transported outward from the Io torus in the equatorial plasma sheet, which we estimated to be typically $\sim 1000 \mathrm{~kg} \mathrm{~s}^{-1}$ in the discussion in the Introduction. Assuming that $\dot{M}$ is a constant in the absence of other significant plasma sources or sinks, and introducing Eq. (9), we find the "Hill-Pontius" equation for the angular velocity profile

$\frac{1}{\rho_{e}} \frac{d}{d \rho_{e}}\left(\rho_{e}^{2} \frac{\omega}{\Omega_{J}}\right)=\frac{8 \pi \Sigma_{P}^{*} F_{e}\left|B_{z e}\right|}{\dot{M}}\left(1-\frac{\omega}{\Omega_{J}}\right)$,

where again we note that $\Sigma_{P}^{*}$ in general varies with position. This is a central topic to which we now turn in the next section.

\section{Dependence of the Pedersen conductivity on the field- aligned current}

Recent modelling work presented by Millward et al. (2002) has investigated how the height-integrated conductivities in Jupiter's auroral zones are enhanced by auroral electron precipitation. In their model runs the precipitating electrons were taken to be a monoenergetic electron beam with energy ranging from 1 to $100 \mathrm{keV}$, thus spanning the range expected from the initial studies of Cowley and Bunce (2001) and Cowley et al. (2002, 2003). In their paper, results are shown for two cases of electron flux, one for a fixed flux of $6.25 \times 10^{12} \mathrm{~m}^{-2} \mathrm{~s}^{-1}$ (corresponding to a current density of
$1 \mu \mathrm{A} \mathrm{m}^{-2}$ ), their Fig. 7, the other for a fixed energy flux of $10 \mathrm{~mW} \mathrm{~m}^{-2}$, their Fig. 8 (we note that in their paper the units of the fixed flux are misprinted as $\mathrm{cm}^{-2} \mathrm{~s}^{-1}$ ). The empirical conductivity models employed here are based on the results shown in these two figures (kindly provided in digital form by G. H. Millward, personal communication, 2003).

Millward et al.'s (2002) results show that, for a given value of the number flux, the enhancement in the Pedersen conductivity is dependent on the precipitating electron energy, strongly peaking in the energy band between $\sim 50$ to $\sim 80 \mathrm{keV}$, where the electrons deposit most of their energy within the Pedersen layer. Electrons of significantly higher energies deposit their energy too low down in the atmosphere to significantly affect the conductivity. In order to estimate the conductivity enhancement associated with a given fieldaligned current (and hence number flux) we thus also need to estimate the energy of the precipitating electrons. To do this we employ the kinetic theory of Knight (1973), as in previous related studies, who showed that for a given ionospheric field-aligned current density $j_{\| i}$, the required minimum fieldaligned accelerating voltage is given by

$e \Phi=W_{t h}\left[\left(\frac{j_{\| i}}{j_{\| i o}}\right)-1\right] \simeq W_{t h}\left(\frac{j_{\| i}}{j_{\| i o}}\right)$,

while the precipitating energy flux is

$E_{f}=\frac{E_{f o}}{2}\left[\left(\frac{j_{\| i}}{j_{\| i o}}\right)^{2}+1\right] \simeq \frac{E_{f o}}{2}\left(\frac{j_{\| i}}{j_{\| i o}}\right)^{2}$,

the latter formula being first derived by Lundin and Sandal (1978). In these expressions $j_{\| i o}$ is the maximum fieldaligned current that can be carried by precipitating magnetospheric electrons without field-aligned acceleration, corresponding to a full downward-going loss cone and an empty upward-going loss cone. This is given by

$j_{\| i o}=e N\left(\frac{W_{t h}}{2 \pi m_{e}}\right)^{1 / 2}$,

where $N$ is the electron number density (assumed to be isotropic), $W_{t h}$ is the electron thermal energy, and $E_{f o}$ is the corresponding precipitating energy flux, given by

$E_{f o}=2 N W_{t h}\left(\frac{W_{t h}}{2 \pi m_{e}}\right)^{1 / 2}$.

The approximations in Eqs. (15) and (16) correspond to the case where $j_{\| i} \gg j_{\| i o}$, as will generally be satisfied in the middle magnetosphere, as discussed previously by Cowley and Bunce (2001) and Cowley et al. (2002, 2003). In this case, therefore, the electron population will indeed form an essentially monoenergetic beam at high altitudes, because $e \Phi \gg W_{t h}$. Consequently, the results presented by Millward et al. (2002) indicate that the ionospheric conductivity will be strongly enhanced when the field-aligned current passes through values such that the accelerating voltage lies in the range $\sim 50$ to $80 \mathrm{keV}$, and will be significantly smaller outside this range. However, judging from the case of the Earth (see 
e.g. Paschmann et al., 2002, chapter 4), the monoenergetic beam will be strongly scattered by wave-particle interactions underneath the accelerating region, forming a broad distribution covering a wide range of energies, thus smoothing the variation of conductivity with current. This seems the most likely scenario at the present time, and is the case which will be assumed here.

Specifically, we have assumed that the auroral electron distribution is isotropic over the downward-going hemisphere (due to the large increase in field strength between the acceleration region at a few $R_{J}$ altitude and the top of the ionosphere), and is given as a function of electron velocity $v$ by

$f(v)=\frac{f_{o}}{\left[\left(\frac{v}{v_{o}}\right)^{\alpha}+\left(\frac{v}{v_{o}}\right)^{\beta}\right]}$,

such that for $\beta>\alpha$, the distribution varies as $v^{-\alpha}$ for $v<v_{o}$, and $v^{-\beta}$ for $v>v_{o}$. The velocity where the spectrum "breaks" between exponents $\alpha$ and $\beta$ is taken to be given by the accelerating voltage $\Phi$, i.e.

$\frac{m_{e} v_{o}^{2}}{2}=e \Phi$,

such that the population with slope $\alpha$ for $v<v_{o}$ corresponds to degraded primary particles, while that with slope $\beta$ for $v>v_{o}$ corresponds to a steeply falling high energy tail produced by the wave-particle interactions. The value of $f_{o}$ is determined by the requirement that the downward-going particles carry current $j_{\| i}$, i.e.

$f_{o}=\left(\frac{j_{\| i}}{\pi e}\right) / \int_{0}^{\infty} \frac{d v v^{3}}{\left[\left(\frac{v}{v_{o}}\right)^{\alpha}+\left(\frac{v}{v_{o}}\right)^{\beta}\right]}$,

and, for the examples employed here, for a given value of $\beta$ we have determined the value of $\alpha$ such that the total precipitating energy flux is just that given by Eq. (16). Example distributions are shown in Figs. $3 \mathrm{a}$ and $3 \mathrm{~b}$ for the cases $\alpha=0, \beta=10$ and $\alpha=2, \beta=8$, respectively. In each case we show the distributions (plotted versus electron energy $W_{e}=m_{e} v^{2} / 2$ in keV) corresponding to both $j_{\| i}=0.1 \mu \mathrm{A} \mathrm{m}^{-2}$ and $1.0 \mu \mathrm{A} \mathrm{m}^{-2}$, where the magnetospheric "parent" population has a density $N=0.01 \mathrm{~cm}^{-3}$ and a thermal energy $W_{e}=2.5 \mathrm{keV}$, these values being based on Voyager data presented by Scudder et al. (1981), as also employed in previous related studies. In this case, the limiting current in Eqs. (15) and (16) is $j_{\| i o} \simeq 0.0134 \mu \mathrm{A} \mathrm{m}^{-2}$, while the limiting energy flux is $0.067 \mathrm{~mW} \mathrm{~m}^{-2}$.

To estimate the enhancement in the height-integrated Pedersen conductivity produced by such precipitating electron distributions we have calculated the precipitating number flux $\mathcal{F}_{n}$ in each of ten contiguous energy bands spanning the energy range from 5 to $105 \mathrm{keV}$, centred on the "monoenergetic" energy values of $10,20, \ldots 100 \mathrm{keV}$ employed by Millward et al. (2002). We have then taken the Pedersen conductivity values determined by Millward et al. (2002)

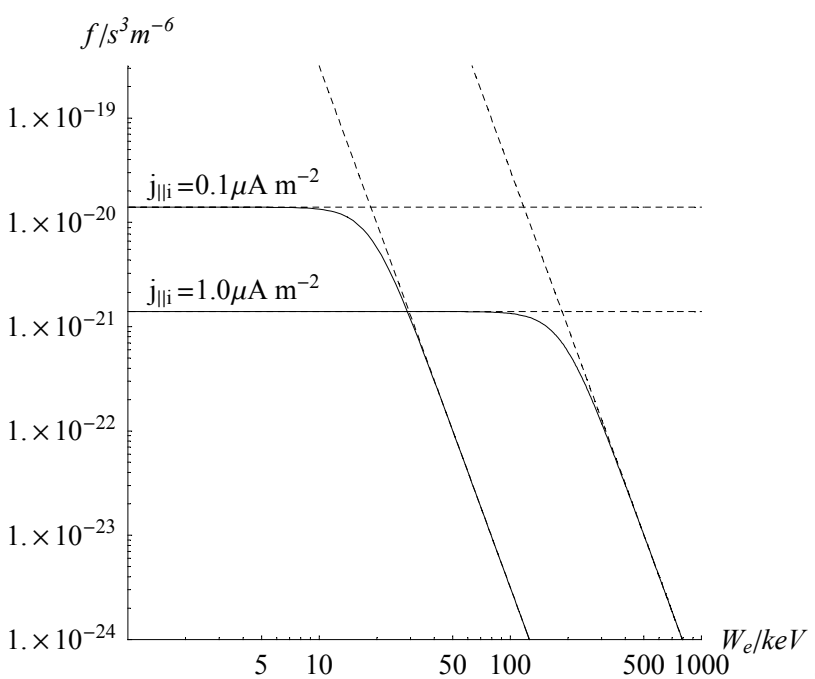

(a)

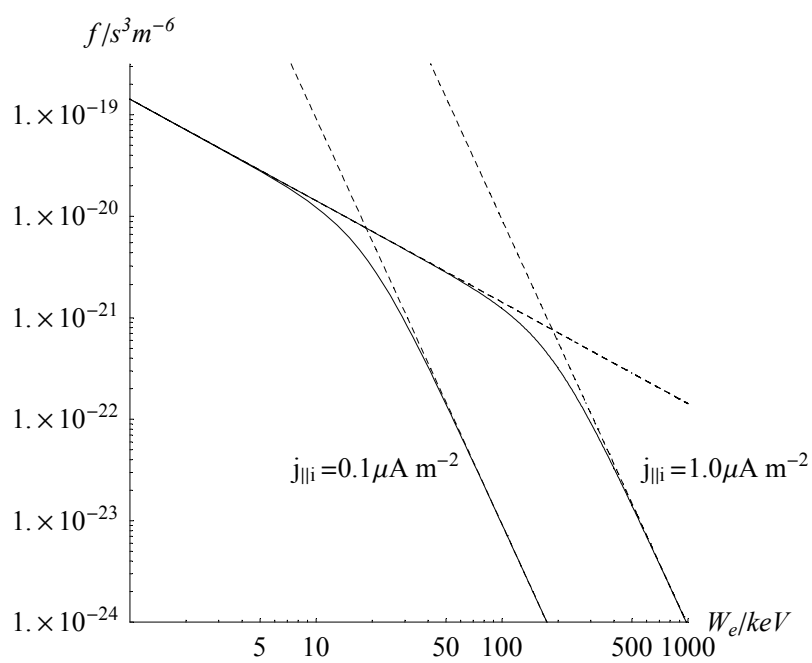

(b)

Fig. 3. Two examples of auroral electron distribution functions, plotted versus electron energy $W_{e}=m_{e} v^{2} / 2$ in $\mathrm{keV}$, determined from Eqs. (19)-(21), employed here with $N=0.01 \mathrm{~cm}^{-3}$ and $W_{t h}=2.5 \mathrm{keV}$. Plot (a) shows the case for $\alpha=0$ and $\beta=10$, while plot (b) shows the case for $\alpha=2$ and $\beta=8$. In both plots the distributions are shown for $j_{\| i}=0.1 \mu \mathrm{A} \mathrm{m}^{-2}$ and $1.0 \mu \mathrm{A} \mathrm{m}^{-2}$. The dashed lines show the asymptotes of the respective power law variations.

for a fixed number flux $\mathcal{F}_{o}=6.25 \times 10^{12} \mathrm{~m}^{-2} \mathrm{~s}^{-1}$ at each energy $\Sigma_{P n}$, and have summed the contributions of each energy band according to

$\Sigma_{P}=\sum_{n=1}^{10} \Sigma_{P n}\left(\frac{\mathcal{F}_{n}}{\mathcal{F}_{o}}\right)^{\gamma_{n}}$

The exponent $\gamma_{n}$ has been determined by comparing the conductivity values determined at fixed number flux shown in Millward et al.'s (2002) Fig. 7, with those determined at fixed energy flux in their Fig. 8. The values of $\gamma_{n}$ so determined vary from $\sim 0.5$ at the lower energies $\sim 10-20 \mathrm{keV}$, to $\sim 0.8$ at the higher energies $\sim 70-80 \mathrm{keV}$. The rationale for employing a power law variation of $\Sigma_{P n}$ with the number flux at fixed 
energy, while linearly summing the contributions of differing energy bands, rests on the fact that electrons of differing energy produce their ionisation at differing heights through the ionosphere, as can be seen in Millward et al.'s (2002) results, and are thus additive to a lowest approximation. While we thus believe that our procedure makes reasonable use of existing information, we nevertheless recognise that it represents a rather crude approximation.

The solid lines in Fig. 4 show how $\Sigma_{P}$ depends on $j_{\| i}$ for four model auroral distribution functions ( $\alpha, \beta$ values), for a hot magnetospheric source electron population with the above values of $N$ and $W_{t h}$. It can be seen that the curves are reasonably similar, rising from small values for small currents, to values of $\sim 2$ mho for $j_{\| i} \sim 0.4 \mu \mathrm{A} \mathrm{m}^{-2}$ (a slope of $\left.\sim 5 \mathrm{mho}\left(\mu \mathrm{A} \mathrm{m}^{-2}\right)^{-1}\right)$. For larger currents, the behaviour of the conductivity depends rather more on the assumed spectrum, specifically on the slope at small energies, below that produced by the voltage drop. However, rather than employing these curves directly, which would be impractical in the numerical integrations which follow, we have instead employed a simple analytical form taken to be representative of these results. This is given, for $j_{\| i}>0$, by

$$
\begin{gathered}
\Sigma_{P}\left(j_{\| i}\right)=0.16 j_{\| i}+\left\{2.45\left[\frac{\left(j_{\| i} / 0.075\right)^{2}}{1+\left(j_{\| i} / 0.075\right)^{2}}\right]\right. \\
\left.\times \frac{1}{\left[1+\exp \left(-\left(j_{\| i}-0.22\right) / 0.12\right)\right]}\right\},
\end{gathered}
$$

where $\Sigma_{P}$ is in mho and $j_{\| i}$ is in $\mu \mathrm{Am}^{-2}$. This form is shown by the dashed line in Fig. 4.

It should be realised that the results shown in Fig. 4 are appropriate only to the above values of the magnetospheric hot electron source parameters, and that differing dependencies of the conductivity on the current will be appropriate to other values. In general, therefore, the conductivity will be a function of both the field-aligned current and the position (i.e. the flux function). For simplicity, however, and in the absence of any established model of the properties of the hot magnetospheric electrons, we have taken the source parameters to be constant throughout the middle magnetosphere, as seems appropriate to an initial investigation. We have thus employed a fixed conductivity model $\Sigma_{P}=\Sigma_{P}\left(j_{\| i}\right)$, independent of position, in all the solutions derived in this paper. Two further points should also be made. The first is that the quantity $\Sigma_{P}$ derived above and shown in Fig. 4 is our estimate of the true value of the height-integrated Pedersen conductivity, while the value required by the theory in Sect. 2 is the "effective" value, reduced from the true value by atmospheric slippage. This is related to the true value by Eqs. (6) and (7), thus depending on parameter $k$. The value of $k$ is not well known at present, but recent work with the JIM model indicates that $k \sim 0.5$ for large current values of $\sim 1 \mu \mathrm{A} \mathrm{m}^{-2}$ (G. H. Millward, personal communication, 2003). Application of Eq. (23) in Sect. 5 is thus modified by the multiplication of the true conductivity by the factor $(1-k)$, where we assume that $k \sim 0.5$. Second, noting that the analytical form Eq. (23) goes to zero as $j_{\| i} \rightarrow 0$, we also add a small constant value $\sim 0.05 \mathrm{mho}$, representing the residual conductivity (produced, for example, by solar illumination) in the absence of precipitation. Further details will be given below.

\section{Solutions for near-linear variations of the conductivity}

Rather than proceed directly to the discussion of solutions tailored specifically to Jovian conditions, employing the empirical conductivity model given by Eq. (23), in this section we first discuss the results of a somewhat wider investigation using simplified conductivity models, which provide insight into the nature of the solutions and their relation to those derived in earlier studies using constant conductivity.

\subsection{Method of obtaining solutions}

We first discuss the method adopted to obtain the solutions required. In the case where $\Sigma_{P}^{*}$ is considered to be a constant, as discussed in previous papers, the equation to be solved is the Hill-Pontius equation, Eq. (14). This is a first order linear equation for $\omega$, which can be solved numerically with the use of one initial or boundary condition, i.e. with the arbitrary choice of the value of $\omega$ at one particular position $\rho_{e}$. However, for a given position $\rho_{e}$, there is only one choice for $\omega$ which satisfies the physical requirement that the plasma near-rigidly corotates at small radial distances, the latter corresponding to the solutions first derived by Hill (1979) and Pontius (1997). All other solutions diverge at small $\rho_{e}$, those starting with a smaller value of $\omega$ eventually diverging to large negative values, while those starting with a larger value of $\omega$ diverging to large positive values. Some examples of such divergent solutions are shown in the Appendix of the paper by Cowley and Bunce (2003). The required solution which does not diverge at the origin can then be found by iterating the value of $\omega$ at the "boundary" position.

If we now consider the variable conductivity problem in which $\Sigma_{P}^{*}$ depends on $j_{\| i}$ according to some model such as Eq. (23), then two equations must be solved simultaneously, i.e. Eq. (12) for the parallel current, and the Hill-Pontius equation Eq. (14) for the plasma angular velocity, which now depends on the parallel current through the dependence of $\Sigma_{P}^{*}$. We thus must solve two coupled first order equations for $j_{\| i}$ and $\omega$, thus requiring the choice of two boundary or initial conditions. These choices are conveniently the values of $j_{\| i}$ and $\omega$ at a given point, $\rho_{e}$, taken throughout here to be the outer boundary of the model at $100 R_{J}$. The choice of $j_{\| i}$ at the outer boundary also fixes the value of $\Sigma_{P}^{*}$ at the boundary, of course, through the chosen conductivity model. For a given value of $j_{\| i}$ (and $\Sigma_{P}^{*}$ ) at the outer boundary we then iterate $\omega$ to find the physically acceptable solution which near-rigidly corotates at small radial distances, using the divergent behaviour of the Hill-Pontius equation at small radial distances outlined above, which applies here also to the 


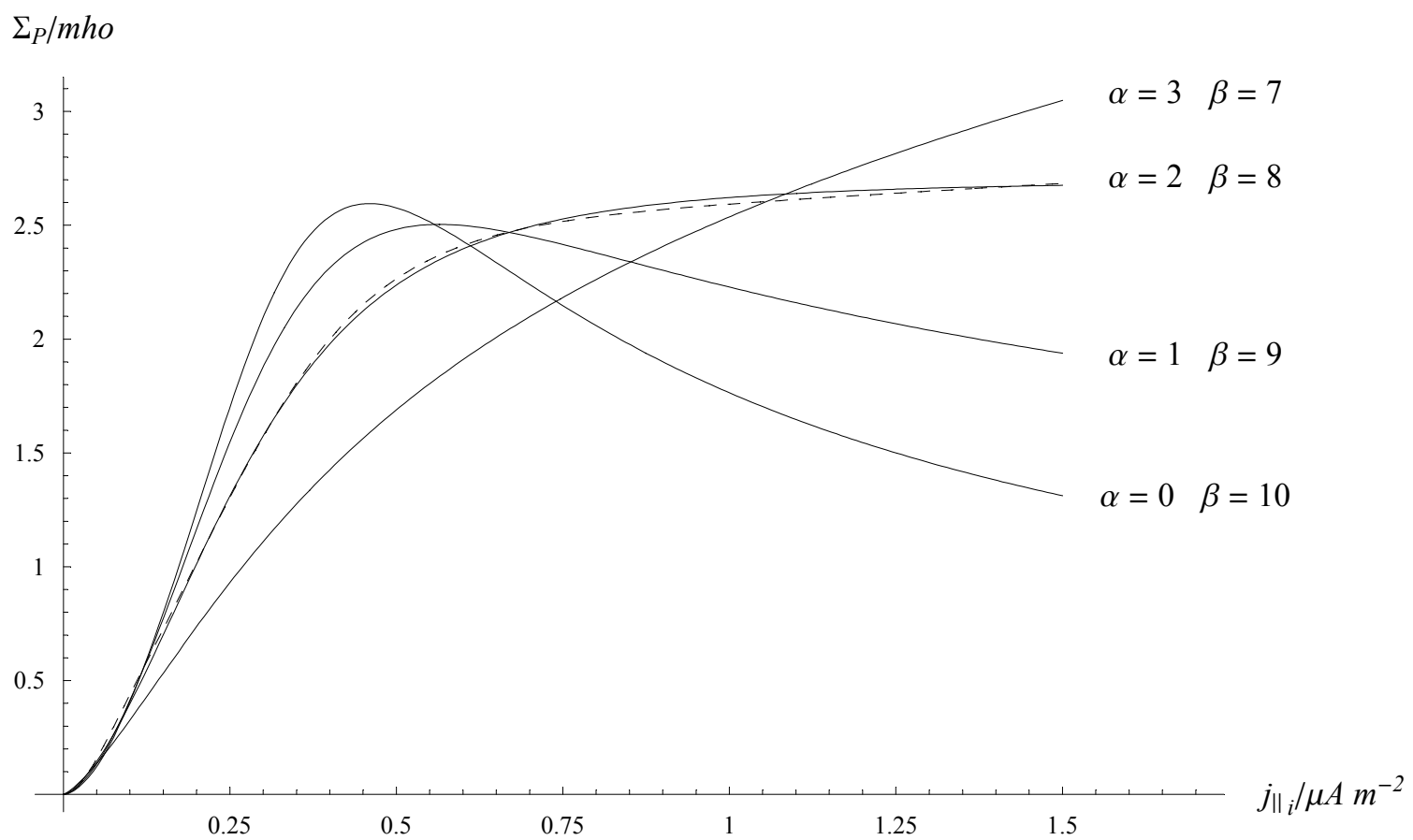

Fig. 4. Plots of the height integrated Pedersen conductivity $\Sigma_{P}$ in mho versus ionospheric field-aligned current density $j_{\| i}\left(\mu \mathrm{A} \mathrm{m}^{-2}\right)$ using four models of the auroral electron distribution functions. The respective $\alpha$ and $\beta$ values are indicated on the plot. The results are based on Millward et al.'s (2002) computations, using Eq. (22). The dashed line shows the analytic form given by Eq. (23).

general problem. It is found that $\omega$ must generally be specified to very many decimal places at $100 R_{J}$, in order to follow the required solution in towards the quasi-dipolar inner magnetosphere without diverging either to large positive or negative values. In practice this procedure has typically been used to track the required solution in from $100 R_{J}$ to $\rho_{e} \sim 10$ to $20 R_{J}$, the solution then being completed by the use of an approximation which is appropriate to the inner region, as we now discuss.

\subsection{Inner region approximations}

The behaviour of the physically required solutions in the inner region where the plasma near-rigidly corotates has been discussed previously by Cowley et al. (2003) and Nichols and Cowley (2003) for the case of constant conductivity, and is now applied here to the more general case. If we put $\omega \approx \Omega_{J}$ into the LHS of Eqs. (13) or (14), we then derive the amount of (small) slippage from rigid corotation required to drive the current $i_{\rho}$ which maintains near-rigid corotation. This yields from Eq. (14)

$$
\frac{\omega}{\Omega_{J}} \simeq 1-\frac{\dot{M}}{4 \pi \Sigma_{P}^{*} F_{e}\left|B_{z e}\right|},
$$

valid for the situation where $\left(1-\omega / \Omega_{J}\right) \ll 1$. Hence, from Eqs. (10) and (12) we find

$$
I_{\rho}=\left(\frac{2 \dot{M} \Omega_{J}}{\left|B_{z e}\right|}\right),
$$

and

$$
j_{\| i}=-\frac{\dot{M} \Omega_{J} B_{J}}{\pi \rho_{e}\left|B_{z e}\right|^{3}} \frac{d\left|B_{z e}\right|}{d \rho_{e}} .
$$

We thus find, as in previous studies, that the currents in the innermost region depend only on $\dot{M}$ and the model of the poloidal field, and not on $\Sigma_{P}^{*}$ (or the model dependence of $\Sigma_{P}^{*}$ on $j_{\| i}$ ), though, of course, the radial extent of the region to which Eqs. (25) and (26) apply does depend on $\Sigma_{P}^{*}\left(j_{\| i}\right)$, as we will find. However, the plasma angular velocity in the inner region then does depend on $\Sigma_{P}^{*}\left(j_{\| i}\right)$ (with $j_{\| i}$ determined from Eq. 26), the departure from rigid corotation varying inversely with the conductivity.

It will be noted from Eq. (26) that $j_{\| i}$ approaches small values in the inner region, being given by

$j_{\| i} \simeq \frac{3 \dot{M} \Omega_{J}}{\pi B_{J}} \rho_{e}^{4}$,

where the field is quasi-dipolar $\left(\left|B_{z e}\right| \approx B_{J} R_{J}^{3} / \rho_{e}^{3}\right)$. The ionospheric conductivity thus perforce approaches the small constant value $\Sigma_{P}^{*}=\Sigma_{P}^{*}\left(j_{\| i}=0\right)$ in the inner region. An additional iterative approach to approximation in the inner region can then be useful in cases where the conductivity does not vary strongly with the parallel current. We can then first solve the Hill-Pontius equation using the constant conductivity $\Sigma_{P}^{*}\left(j_{\| i}=0\right)$, from which an approximation to $j_{\| i}$ is derived, which can be used to then derive a varying conductivity profile from the model for $\Sigma_{P}^{*}\left(j_{\| i}\right)$, which is an explicit function of position. This can then be used to solve 


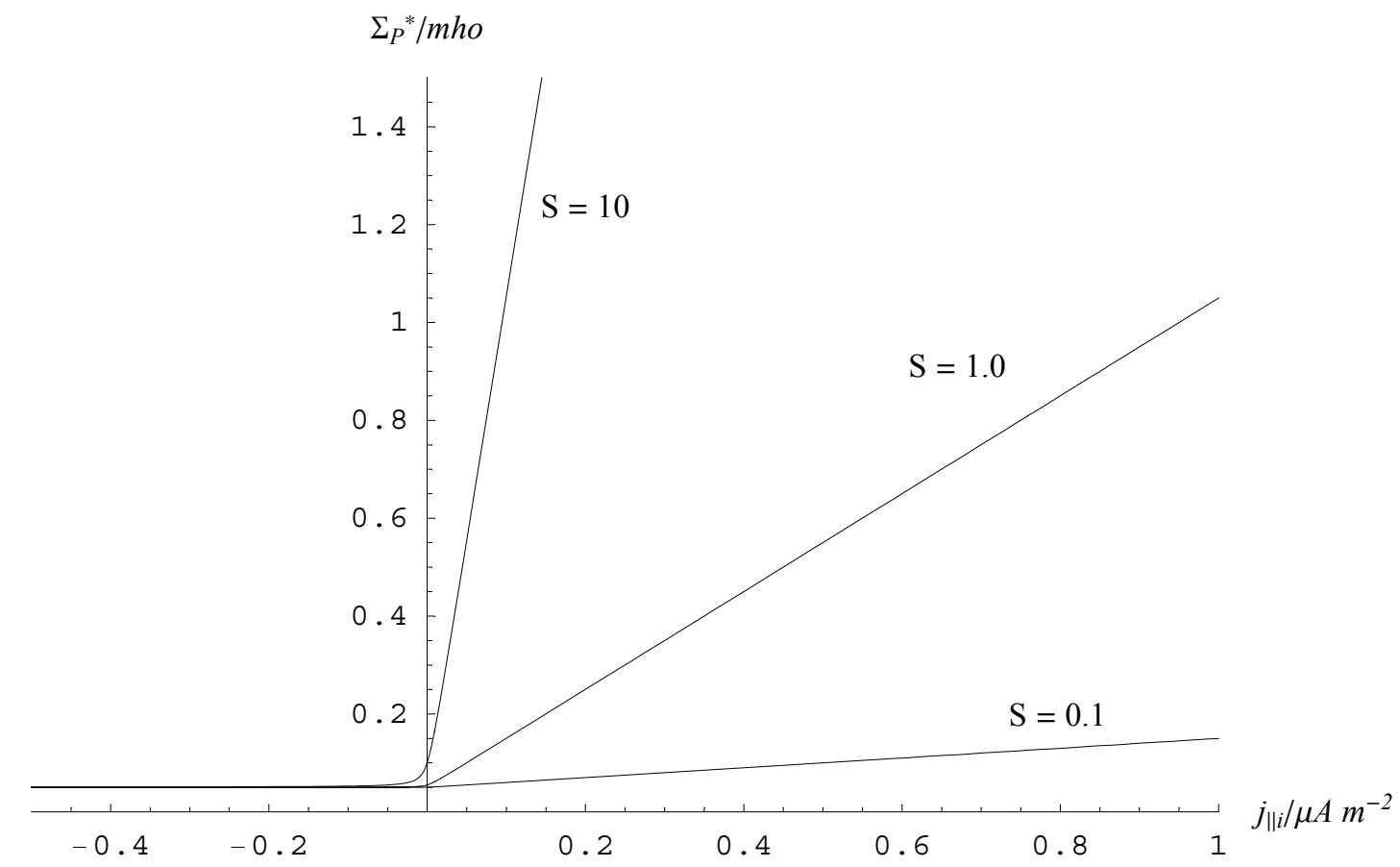

Fig. 5. Plots of the effective height integrated Pedersen conductivity $\Sigma_{P}^{*}$ in mho versus ionospheric field-aligned current density $j_{\| i}\left(\mu \mathrm{A} \mathrm{m}^{-2}\right)$ using the near-linear conductivity model given by Eq. (28). Three cases are shown for $S=0.1,1$, and $10 \mathrm{mho}(\mu \mathrm{A} \mathrm{m})^{-1}$, as indicated.

the Hill-Pontius equation again, yielding a "first iteration" to the angular velocity and currents, from which a modified varying conductivity profile is derived. In principle, this procedure can then be repeated to find successive iterations to the solution in the inner region.

\subsection{Results for a near-linear conductivity model}

We now present results, derived as discussed above, for a simplified conductivity model in which the Pedersen conductivity has a small near-constant value for $j_{\| i}$ negative (i.e. downward field-aligned current), while increasing nearlinearly with the field-aligned current density when $j_{\| i}$ is positive (i.e. upward field-aligned current). Specifically, the function employed is

$\Sigma_{P}^{*}\left(j_{\| i}\right)=\Sigma_{P o}^{*}+\left(\frac{S}{2}\right)\left(\sqrt{j_{\| i}^{2}+j_{\| i}^{* 2}}+j_{\| i}\right)$,

which is such that $\Sigma_{P}^{*} \simeq \Sigma_{P o}^{*}$ for $j_{\| i} \ll-\left|j_{\| i}^{*}\right|$, and $\Sigma_{P}^{*} \simeq \Sigma_{P o}^{*}+S j_{\| i}$ for $j_{\| i} \gg\left|j_{\| i}^{*}\right|$. The value of the limiting conductivity in the inner region of the system is then

$\Sigma_{P}^{*}\left(j_{\| i}=0\right)=\Sigma_{P o}^{*}+\left(\frac{S j_{\| i}^{*}}{2}\right)$.

In the results presented here we have taken $\Sigma_{P o}^{*}=0.05 \mathrm{mho}$ (motivated, for example, by the results of Hill (1980) obtained from analysis of plasma angular velocity profiles in the innermost region), $j_{\| i}^{*} \simeq 0.01 \mu \mathrm{A} \mathrm{m}^{-2}$ (a somewhat arbitrary small value, which is such that the conductivity varies between the above two behaviours over a narrow range of $j_{\| i} \sim 0.01 \mu \mathrm{A} \mathrm{m}^{-2}$ about $j_{\| i}=0$ ), and three values of the slope $S=0.1,1.0$ and $10 \mathrm{mho}\left(\mu \mathrm{A} \mathrm{m}^{-2}\right)^{-1}$. We show these three models for the conductivity in Fig. 5, plotted versus $j_{\| i}$ over the physically interesting range out to $1 \mu \mathrm{Am}^{-2}$. We note that the initial slopes of the empirical curves for the true value of the Pedersen conductivity (as opposed to the effective value discussed here) shown in Fig. 4, lie between the two larger of these values of $S$. Results for the smallest value, $S=0.1 \mathrm{mho}\left(\mu \mathrm{A} \mathrm{m}^{-2}\right)^{-1}$, are included in order to address the issue of the relation between the solutions derived here and those obtained for constant conductivity in previous studies.

We thus begin here with the latter case, $S=0.1 \mathrm{mho}\left(\mu \mathrm{A} \mathrm{m}^{-2}\right)^{-1}$, and also choose $\dot{M}=1000 \mathrm{~kg} \mathrm{~s}^{-1}$ as a typical value, as indicated above. In Fig. 6 we show results in a standard form that will be used throughout the paper. The four panels of the figure show, respectively, the plasma angular velocity normalised to $\Omega_{J}$, the azimuth-integrated equatorial radial current $I_{\rho}$ in MA, the field-aligned current density at the top of the ionosphere $j_{\| i}$ in $\mu \mathrm{A} \mathrm{m}^{-2}$, and the effective height-integrated Pedersen conductivity $\Sigma_{P}^{*}$ in mho, all plotted versus radial distance $\rho_{e}$. The last two quantities, of course, correspond to the values at the respective feet of the field lines concerned, but they have been plotted here versus $\rho_{e}$ so that the relationships between all these quantities can be most readily appreciated. In the next section we will also show solutions projected into the ionosphere and plotted versus dipole co-latitude, so that the ionospheric distributions can also be appreciated. 


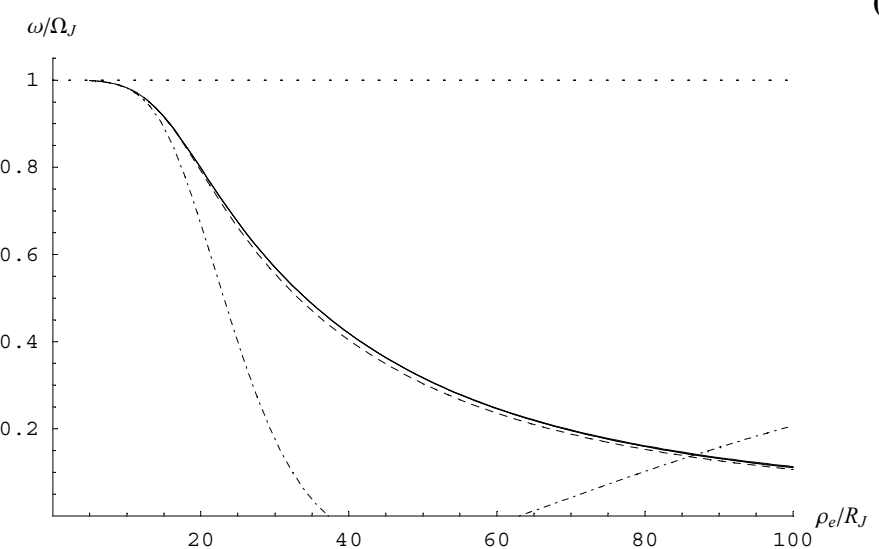

(a)

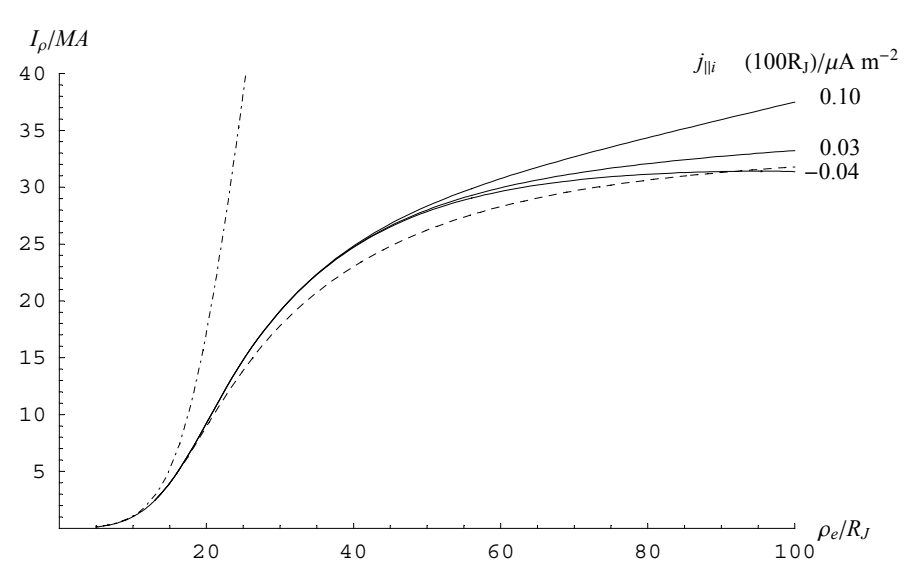

(b)

(c)
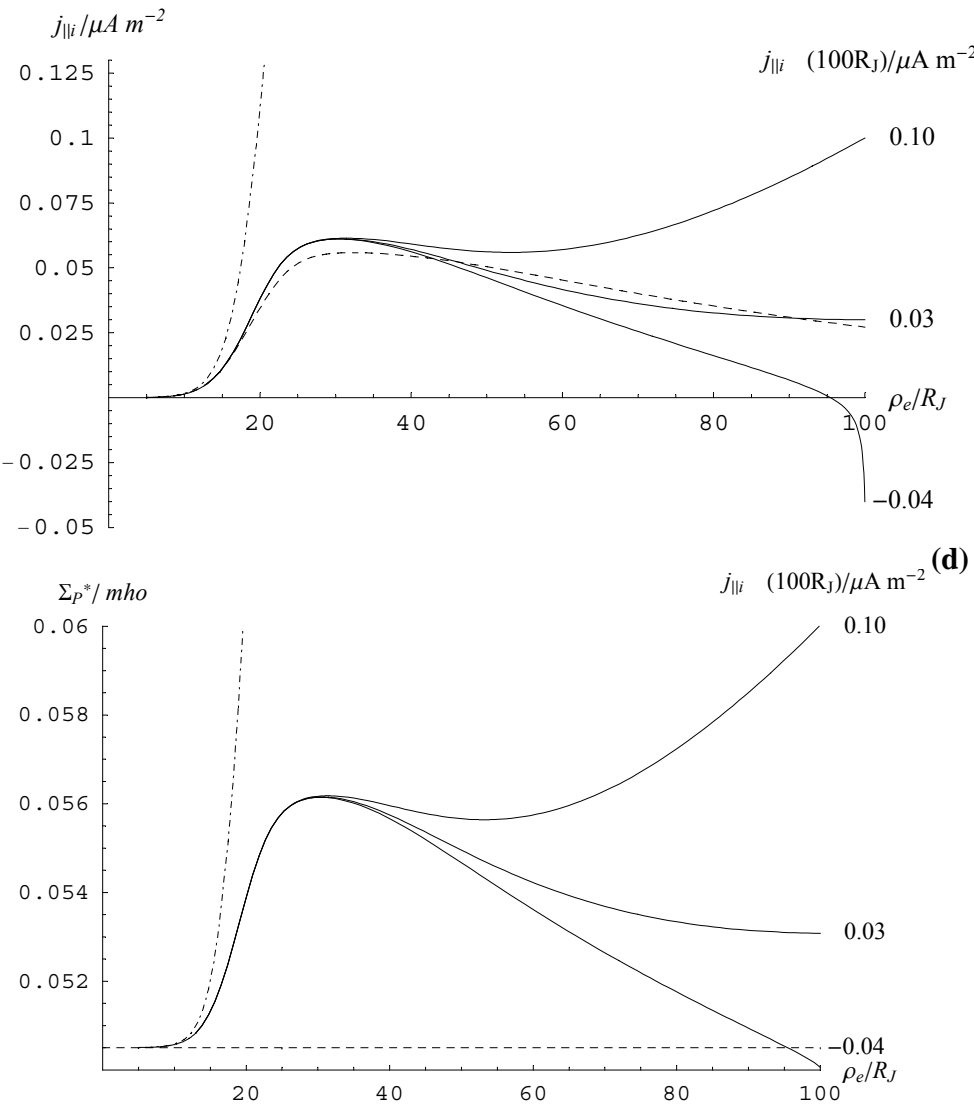

Fig. 6. Plots of the magnetosphereionosphere coupling current system parameters for the near-linear conductivity model (Eq. 28) with slope $S=0.1 \mathrm{mho}\left(\mu \mathrm{A} \mathrm{m}^{-2}\right)^{-1}$ (solid lines), for constant conductivity $\Sigma_{P}^{*}\left(j_{\| i}=0\right)=0.0505$ mho $\quad$ (dashed lines), and the near-rigid corotation approximations given by Eqs. (24) (26) (dot-dashed lines). The three solid lines represent boundary condition choices of $j_{\| i}\left(100 R_{J}\right)=0.10, \quad 0.03$ and $-0.04 \mu \mathrm{A} \mathrm{m}^{-2}$ as indicated. The solutions in each case are tracked to $13 R_{J}$, inside which they are completed by the "first-iteration" approximation discussed in Sect. 4.2. All parameters are plotted versus jovicentric equatorial radial distance $\rho_{e}$. Plot (a) shows the plasma angular velocity $\omega$ normalised to the planet's angular velocity $\Omega_{J}$. The three solid curves are closely similar to one another, and, therefore, are not labelled. Plot (b) shows the azimuth-integrated equatorial radial current $I_{\rho}$ in MA, plot (c) shows the field-aligned current density at the feet of the field lines $j_{\| i}$ in $\mu \mathrm{A} \mathrm{m}^{-2}$, while plot (d) shows the effective heightintegrated Pedersen conductivity $\Sigma_{P}^{*}$ in mho. 
The dot-dashed lines in the figure panels show the small$\rho_{e}$ approximations given by Eqs. (24) to (26), with $\Sigma_{P}^{*}$ evaluated at $j_{\| i}$ given by Eq. (26). These approximations should be valid in the inner region where the departure of the plasma from rigid corotation is small. The dashed lines also show the solution obtained, as in previous works, if the conductivity is taken to be the constant value $\Sigma_{P}^{*}\left(j_{\| i}=0\right)$, equal (from Eq. 29) to 0.0505 mho in this case. According to the above discussion, these are then the curves to which our solutions should asymptote in the inner region as $j_{\| i}$ falls to small values, such that the conductivity falls to $\Sigma_{P}^{*}\left(j_{\| i}=0\right)$. It can be seen that $j_{\| i}$ for this solution peaks at a value of $\sim 0.055 \mu \mathrm{A} \mathrm{m}^{-2}$ at $\sim 30 R_{J}$, and falls to $\sim 0.028 \mu \mathrm{A} \mathrm{m}^{-2}$ at the outer boundary at $100 R_{J}$. We note that such values of $j_{\| i}$ result in only modest increases in the conductivity according to our assumed model, for example, reaching $\sim 0.0562$ mho for $j_{\| i} \sim 0.056 \mu \mathrm{A} \mathrm{m}^{-2}$. If we then choose a value of $j_{\| i}$ at the outer boundary at $100 R_{J}$ which is close to that of the constant conductivity curve, we may expect to derive a closely similar solution, which is shown by the central solid curve in each of the panels in Fig. 6, displaying the solution obtained by the numerical integration of Eqs. (12) and (14), which near-rigidly corotates at small $\rho_{e}$ and has the value $j_{\| i}=0.03 \mu \mathrm{A} \mathrm{m}^{-2}$ at $\rho_{e}=100 R_{J}$. (Note, however, that in Fig. $6 \mathrm{a}$ the solid lines are so close to each other that they are essentially indistinguishable.) It can be seen that, as expected, this solution is very close to the constant conductivity solution over the whole range of distances. The iterated value of $\omega / \Omega_{J}$ at $100 R_{J}$, for example, is $\left(\omega / \Omega_{J}\right) \approx 0.1114$ compared with $\left(\omega / \Omega_{J}\right) \approx 0.1065$ for the constant conductivity solution. We thus confirm that the solutions obtained here reduce to those found previously for constant conductivity in the appropriate limit.

Two other variable conductivity solutions are also shown in Fig. 6, for $j_{\| i}=0.10 \mu \mathrm{A} \mathrm{m}^{-2}$ and $j_{\| i}=-0.04 \mu \mathrm{A} \mathrm{m}^{-2}$ at $\rho_{e}=100 R_{J}$, such that the current at the outer boundary deviates considerably from that of the constant conductivity solution, one to larger positive values, the other to negative values. It can be seen that these solutions are closely similar to that for $j_{\| i}=0.03 \mu \mathrm{A} \mathrm{m}^{-2}$ in the inner region, diverging only at larger distances towards the boundary condition imposed at $100 R_{J}$. If the solution curves are projected (somewhat unphysically) beyond $100 R_{J}$, it is found that the solution for negative $j_{\| i}$ at the boundary diverges to large negative currents at a radial distance just beyond $100 R_{J}$, while the solutions for positive currents at the boundary grow approximately linearly with the distance (results not shown). However, as can be seen in Fig. 6a, the solutions for $\left(\omega / \Omega_{J}\right)$ in all cases remain very close to that for the constant conductivity $\Sigma_{P}^{*}=\Sigma_{P}^{*}\left(j_{\| i}=0\right)=0.505 \mathrm{mho}$, shown by the dashed line.

We now turn to cases with significantly larger values of the slope $S$ in the conductivity model, such that the enhancement of the conductivity with current is by a more substantial factor. For example, for $S=1 \mathrm{mho}\left(\mu \mathrm{A} \mathrm{m}^{-2}\right)^{-1}$ the conductivity increases from 0.055 mho when $j_{\| i}=0$ to 0.150 mho when $j_{\| i}=0.1 \mu \mathrm{A} \mathrm{m}^{-2}$, while for $S=10 \mathrm{mho}\left(\mu \mathrm{A} \mathrm{m}^{-2}\right)^{-1}$ the corresponding increase is from 0.1 to $1.052 \mathrm{mho}$ (see
Fig. 5). Results for these values of $S$ are shown in Figs. 7 and 8 , respectively, where again we take $\dot{M}=1000 \mathrm{~kg} \mathrm{~s}^{-1}$. The format of the figures is similar to Fig. 6 . It can be seen in Figs. 7a and 8a that in the innermost region the plasma angular velocity decreases from near-rigid corotation with increasing distance, in line with expectations based on the constant conductivity solution with $\Sigma_{P}^{*}=\Sigma_{P}^{*}\left(j_{\| i}=0\right)$, shown by the long-dashed line, and the near-rigid approximation Eq. (24), shown by the dot-dashed line. The fieldperpendicular and field-parallel current components shown in Figs. $7 \mathrm{~b}-\mathrm{c}$ and $8 \mathrm{~b}-\mathrm{c}$ correspondingly increase in accordance with Eqs. (25) and (26). However, as the parallel current increases in accordance with Eq. (26), so does the ionospheric Pedersen conductivity in accordance with Eq. (28), such that when the increase in conductivity becomes comparable with or larger than $\Sigma_{P}^{*}\left(j_{\| i}=0\right)$, the plasma angular velocity departs from the constant conductivity solution (long-dashed line), and falls less rapidly with distance, in accordance with Eq. (24) (dot-dashed line). The onset of this behaviour occurs at smaller radial distances for larger values of $S$, and hence at smaller departures of the angular velocity from rigid corotation, as can be seen by comparing Figs. 7a and 8a. Beyond this point, the increase in the conductivity with the parallel current given by Eq. (26) is such that the angular velocity given by Eq. (24) (dotdashed line) shows a shallow minimum, with $\left(\omega / \Omega_{J}\right) \approx 0.85$ at $\rho_{e} \approx 25 R_{J}$ for $S=1 \mathrm{mho}\left(\mu \mathrm{A} \mathrm{m}^{-2}\right)^{-1}$ in Fig. 7a, and with $\left(\omega / \Omega_{J}\right) \approx 0.97$ at $\rho_{e} \approx 15 R_{J}$ for $S=10 \mathrm{mho}\left(\mu \mathrm{A} \mathrm{m}^{-2}\right)^{-1}$ in Fig. 8a, before slowly increasing again at larger distances (the departure from rigid corotation decreasing as $\sim \rho_{e}^{-(m-2)}$ in this regime, where $m$ is the exponent of the KK field model in Eq. 3a). Equations (24) to (26) thus imply that for $S \geq 1 \mathrm{mho}\left(\mu \mathrm{A} \mathrm{m}^{-2}\right)^{-1}$ the enhancement in the ionospheric conductivity can be such that near-rigid corotation conditions (given by Eq. 24) can be maintained to large distances, and with it the growth of the current components according to Eqs. (25) and (26). The numerical results (solid lines) show that this is indeed the case, with the computed solutions for the angular velocity following the near-rigid corotation value given by Eq. (24) (approximately for $S=1 \mathrm{mho}\left(\mu \mathrm{A} \mathrm{m}^{-2}\right)^{-1}$ in Fig. 7a, and very closely for $S=10 \mathrm{mho}\left(\mu \mathrm{Am}^{-2}\right)^{-1}$ in Fig. $8 \mathrm{a}$ ) out to a certain radial distance determined by the outer boundary condition before falling to lower values at larger distances. The distance to which the near-rigid corotation approximation is followed increases with the chosen value of $j_{\| i}$ (and hence $\Sigma_{P}^{*}$ ) at the outer boundary at $100 R_{J}$, as can be seen in the figures. These results therefore confirm the conjecture of Cowley and Bunce (2001) that precipitation-induced enhancements of the Pedersen conductivity can act to maintain the plasma angular velocity closer to rigid corotation to much larger radial distances than anticipated on the basis of previously presented solutions using typical constant "background" Pedersen conductivities of a few tenths of a mho. 


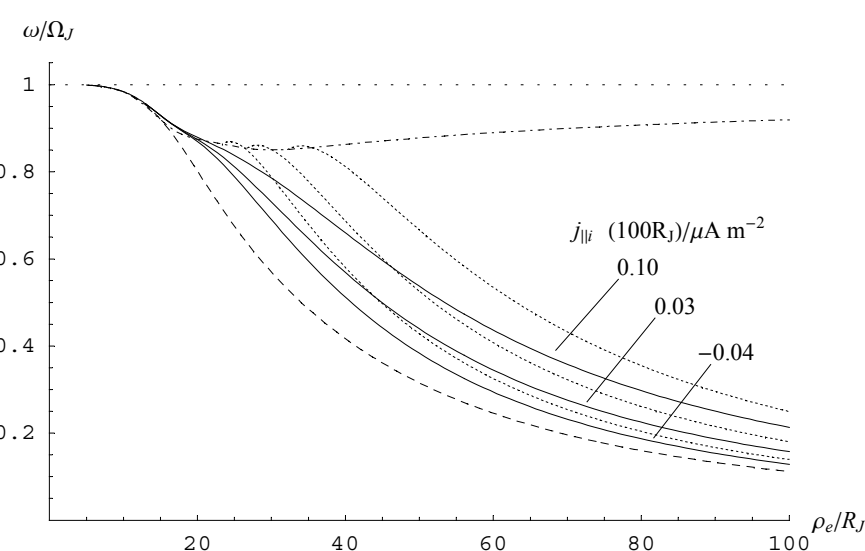

(a)

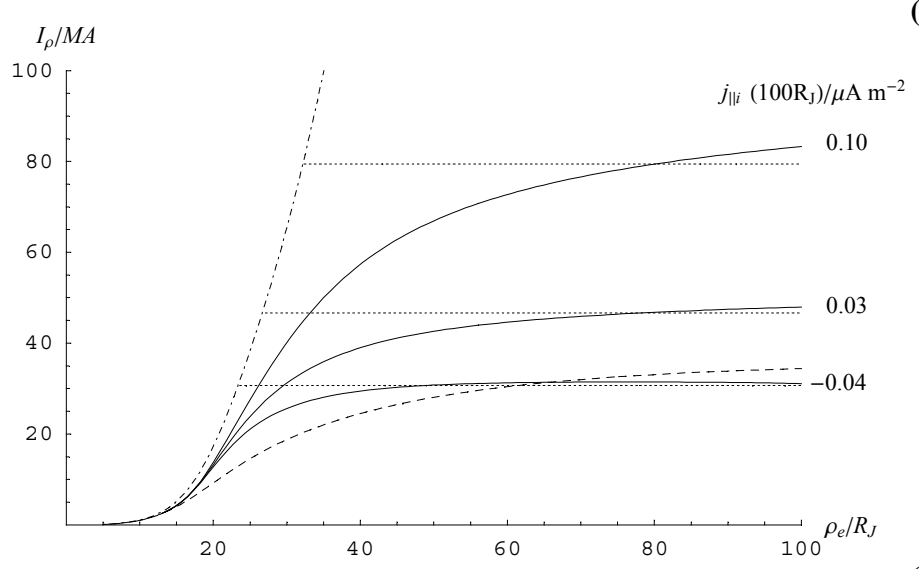

(b)

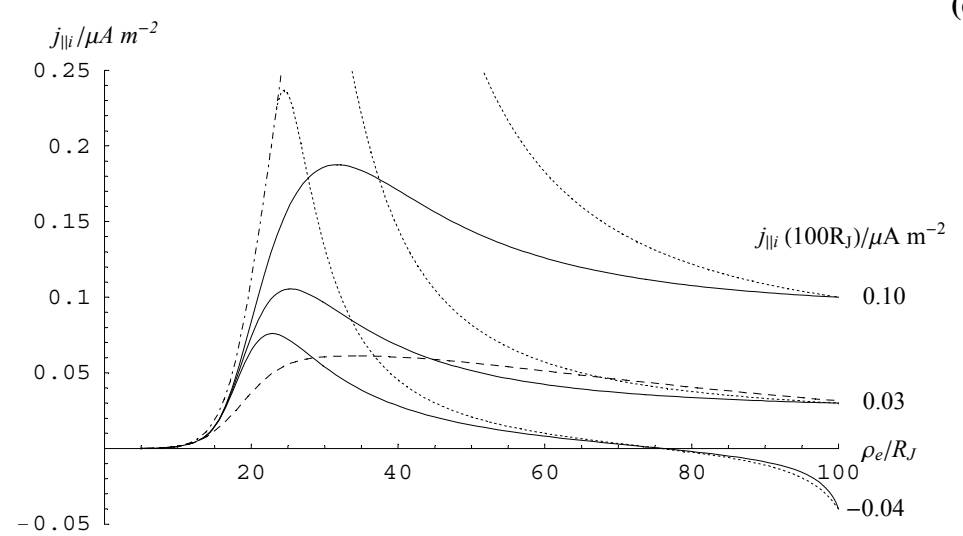

(d)

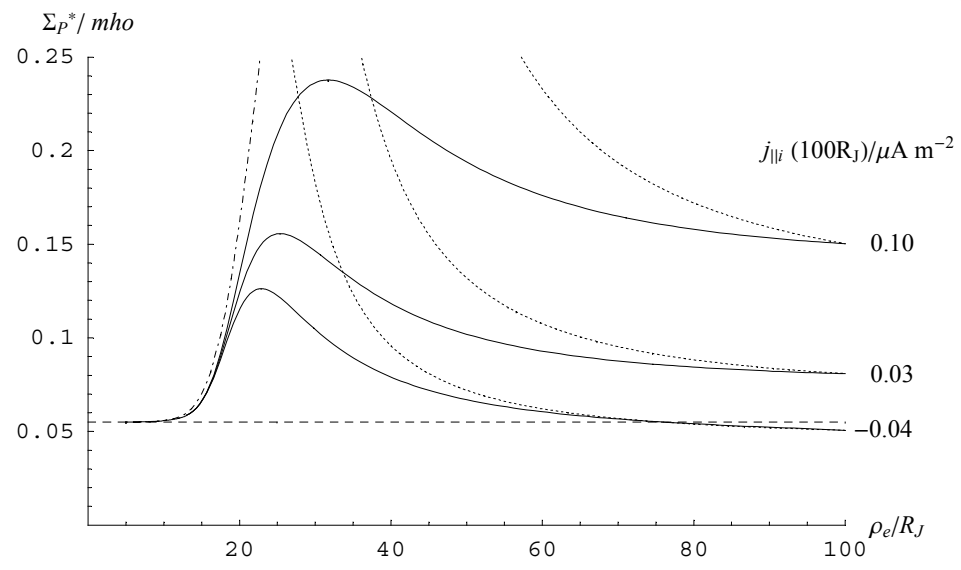

Fig. 7. Plots of the magnetosphereionosphere coupling current system parameters for the near-linear conductivity model (Eq. 28) with slope $S=1.0 \mathrm{mho}\left(\mu \mathrm{A} \mathrm{m}^{-2}\right)^{-1}$ (solid lines), for constant conductivity $\Sigma_{P}^{*}\left(j_{\| i}=0\right)=0.055$ mho $\quad$ (dashed lines), and the near-rigid corotation approximations given by Eqs. (24)(26) (dot-dashed lines). Also shown by the dotted lines is the outer region approximation calculated from Eqs. (30)-(33). The three solid lines represent boundary condition choices of $j_{\| i}\left(100 R_{J}\right)=0.10, \quad 0.03$ and $-0.04 \mu \mathrm{A} \mathrm{m}^{-2}$ as indicated, as in Fig. 6. The solutions in each case are tracked to $13 R_{J}$, inside which they are completed by the "first-iteration" approximation discussed in Sect. 4.2. All parameters are plotted versus jovicentric equatorial radial distance $\rho_{e}$. Plot (a) shows the plasma angular velocity $\omega$ normalised to the planet's angular velocity $\Omega_{J}$, plot (b) shows the azimuth-integrated equatorial radial current $I_{\rho}$ in MA, plot (c) shows the field-aligned current density at the feet of the field lines $j_{\| i}$ in $\mu \mathrm{A} \mathrm{m}^{-2}$, while plot (d) shows the effective heightintegrated Pedersen conductivity $\Sigma_{P}^{*}$ in mho. 


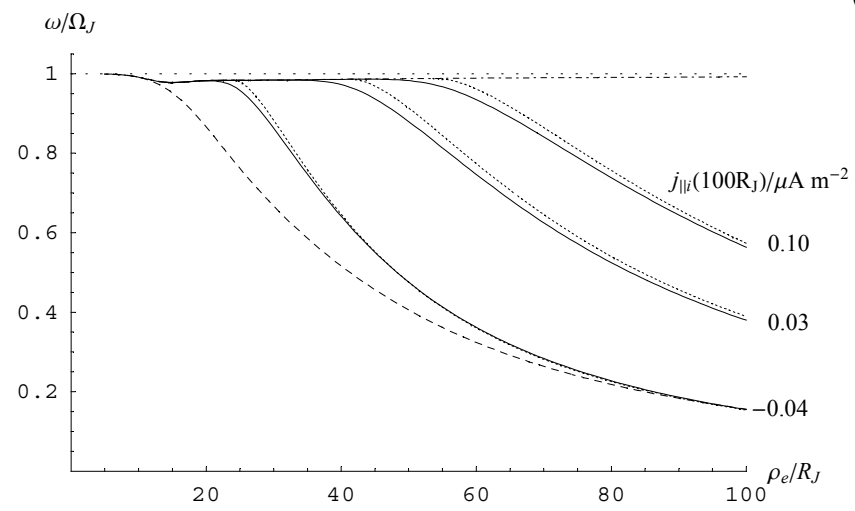

(a)

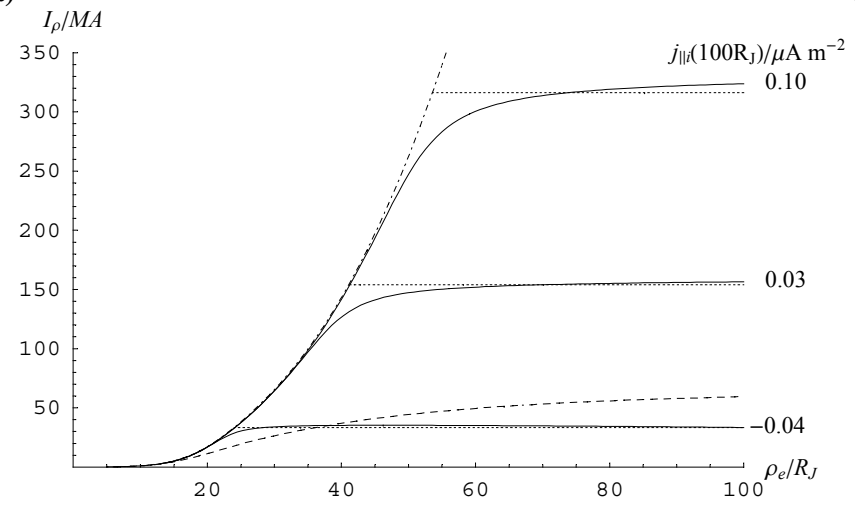

(c)

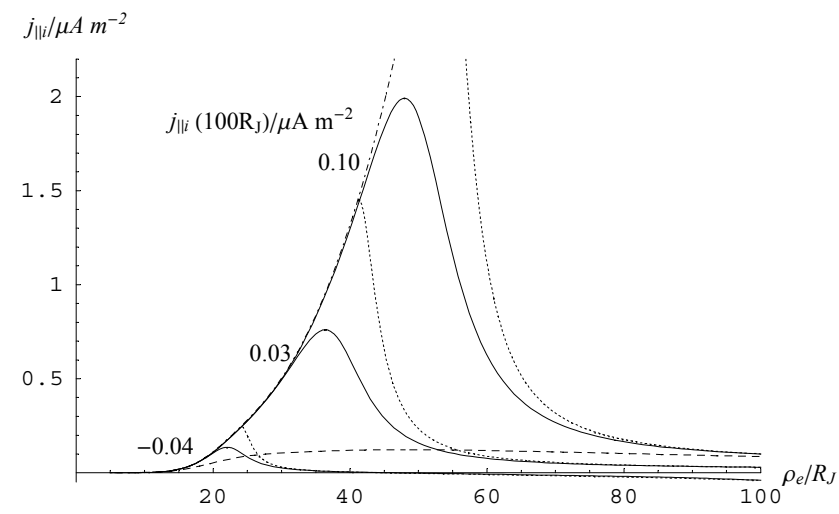

(b)

(d)

Fig. 8. As for Fig. 7 but with $S=10 \mathrm{mho}\left(\mu \mathrm{A} \mathrm{m}^{-2}\right)^{-1}$, such that $\Sigma_{P}^{*}\left(j_{\| i}=0\right)=0.1$ mho.

The corresponding behaviour of the current components shown in Figs. $7 \mathrm{~b}-\mathrm{c}$ and $8 \mathrm{~b}-\mathrm{c}$ is thus that in the inner region they follow the near-rigid corotation approximations given by Eqs. (25) and (26), increasing rapidly with increasing distance depending on $\dot{M}$ and the equatorial magnetic model $\left|B_{z e}\right|$, over the radial range where the angular velocity remains close to the near-rigid corotation approximation Eq. (24). Beyond this distance, where the angular velocity falls away from this behaviour, the field-aligned current also falls away to smaller positive values, rapidly in the case of $S=10 \mathrm{mho}\left(\mu \mathrm{A} \mathrm{m}^{-2}\right)^{-1}$ shown in Fig. $8 \mathrm{c}$, to the value of the chosen boundary condition at $\rho_{e}=100 R_{J}$. Correspondingly, the value of the Pedersen conductivity also falls with distance in the outer region, as seen in Figs. $7 \mathrm{~d}$ and $8 \mathrm{~d}$, while the total current $I_{\rho}$, shown in Figs. $7 \mathrm{~b}$ and $8 \mathrm{~b}$, tends towards a constant value. Overall, it can be seen that in cases where the conductivity increases rapidly with the current density (i.e. $S$ is large), the form of the current profiles differ significantly from those derived previously for constant conductivity. In the latter case the upward field-aligned current density tends to be broadly distributed over the middle magnetosphere current sheet outside of $\sim 20 R_{J}$ for typical parameters (see, e.g. the solutions in Cowley et al. $(2002,2003)$ and Nichols and Cowley, 2003), such that the total radial current grows gradually with increasing distance. In the solutions found here for large $S$, however, the field-aligned current input to the current sheet is instead concentrated in the inner part of the region where the conductivity is also enhanced, and then falls to smaller but still positive values in the region beyond, the radial extent of the main upward field-aligned current region then depending on the outer boundary condition. The total radial current then grows with distance according to Eq. (25) within the main region of field-aligned current in the inner region, while plateauing at almost constant values in the region beyond.

\subsection{Outer region approximations}

Approximate solutions based on these results can then be developed for the outer region, using the governing equations given in Sect. 2. Specifically, we replace Eq. (12) by

$I_{\rho}=8 \pi \Sigma_{P}^{*} F_{e} \Omega_{J}\left(1-\frac{\omega}{\Omega_{J}}\right) \approx$ constant,

in which case the Hill-Pontius equation, in the form given by Eq. (13), can be integrated directly to give

$$
\begin{aligned}
& \left(\frac{\omega}{\Omega_{J}}\right) \approx\left(\frac{\rho_{e}^{\prime}}{\rho_{e}}\right)^{2}\left(\frac{\omega}{\Omega_{J}}\right)^{\prime} \\
& +\frac{I_{\rho}}{\dot{M} \Omega_{J} \rho_{e}^{2}}\left(F_{e}\left(\rho_{e}^{\prime}\right)-F_{e}\left(\rho_{e}\right)\right),
\end{aligned}
$$


$\omega / \Omega_{J}$
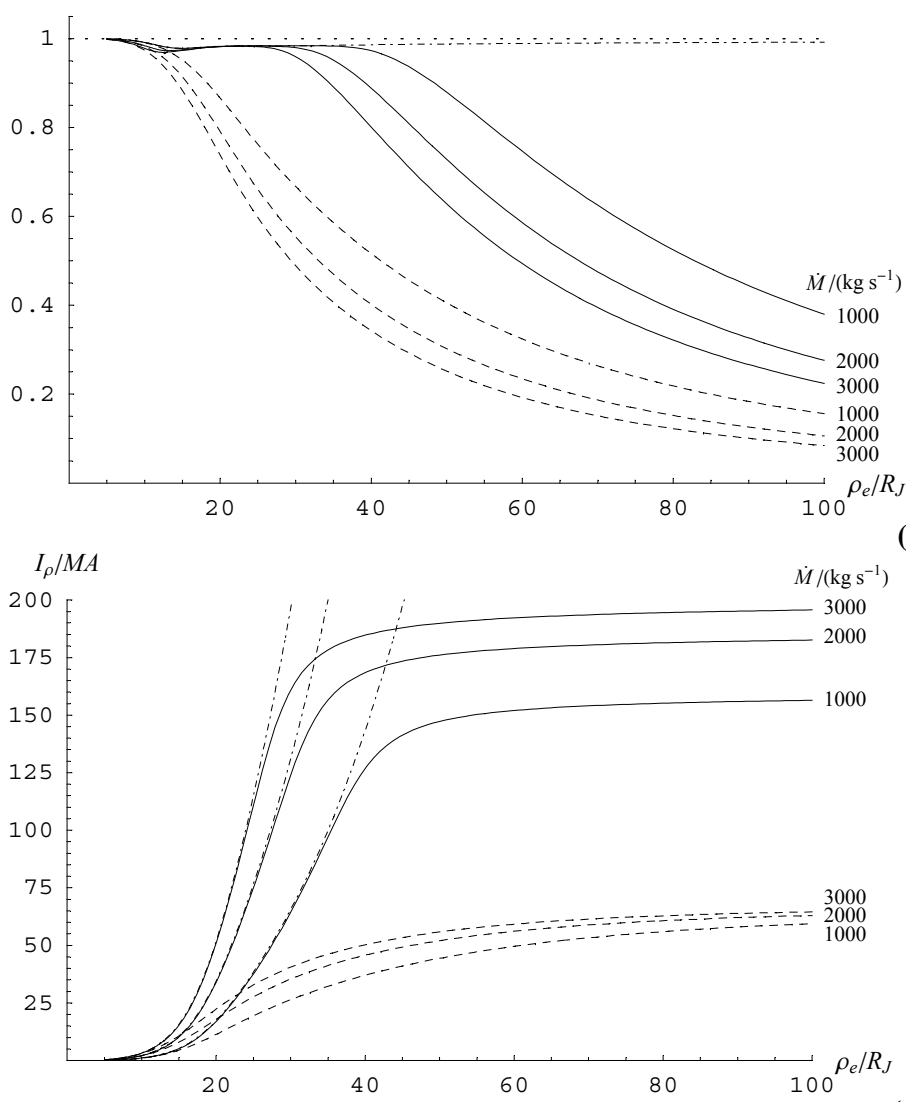

$j_{\| i} / \mu A m^{-2}$

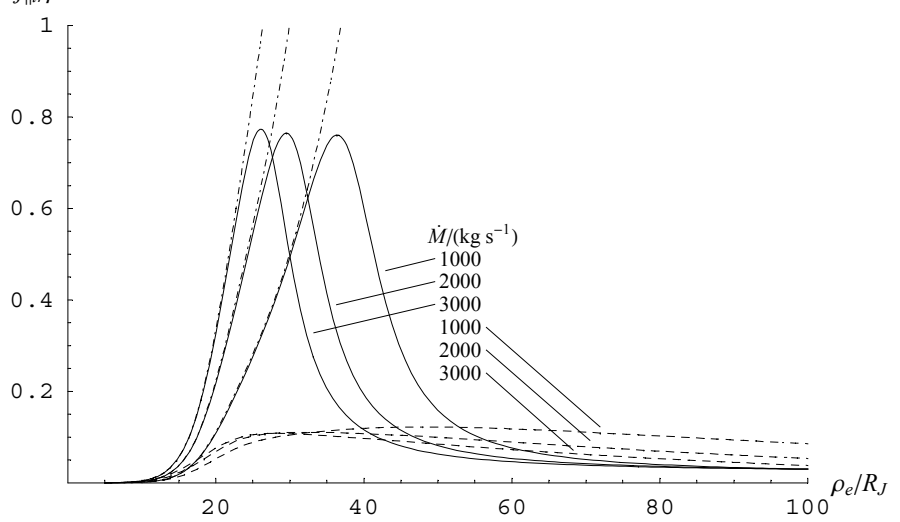

$\Sigma_{P}^{*} / m h o$

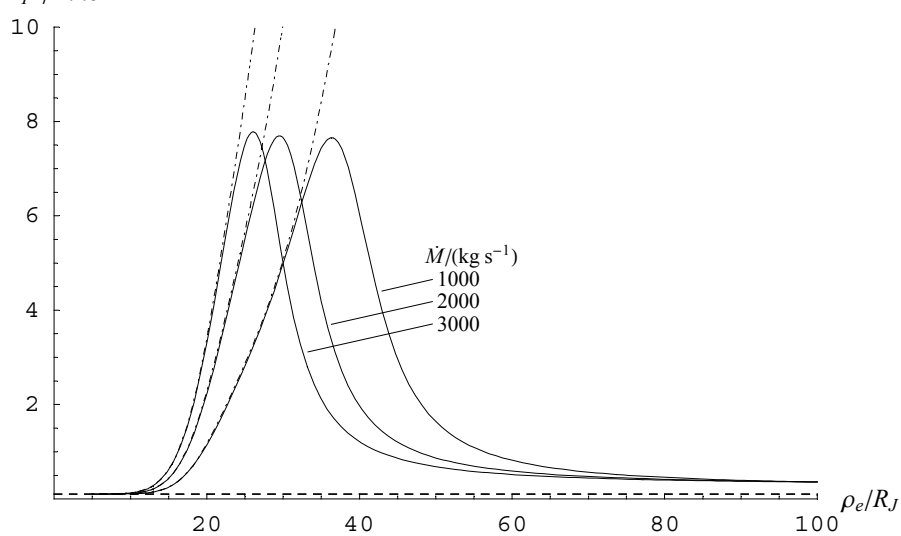

(a)

(b)

Fig. 9. Plots of the magnetosphereionosphere coupling current system parameters in a similar format to Figs. 6-8 for the near-linear conductivity model (Eq. 28) with slope $S=10 \mathrm{mho}\left(\mu \mathrm{A} \mathrm{m}^{-2}\right)^{-1} \quad$ (solid lines), for a constant conductivity $\Sigma_{P}^{*}\left(j_{\| i}=0\right)=0.1$ mho (dashed lines), and the near-rigid corotation approximation given by Eqs. (24)-(26) (dot-dashed lines) for three values of the iogenic plasma mass outflow rate $\dot{M}=1000,2000$ and $3000 \mathrm{~kg} \mathrm{~s}^{-1}$ (as indicated). The solutions in each case are tracked to $13 R_{J}$, inside which they are completed by the near-rigid corotation approximation. All parameters are plotted versus jovicentric equatorial radial distance $\rho_{e}$. Plot (a) shows the plasma angular velocity $\omega$ normalised to the planet's angular velocity $\Omega_{J}$, plot (b) shows the azimuth-integrated equatorial radial current $I_{\rho}$ in MA, plot (c) shows the field-aligned current density at the feet of the field lines $j_{\| i}$ in $\mu \mathrm{A} \mathrm{m}^{-2}$, while plot (d) shows the effective height-integrated Pedersen conductivity $\Sigma_{P}^{*}$ in mho. 
where $\left(\omega / \Omega_{J}\right)^{\prime}$ is a known value of the angular velocity at some radial distance $\rho_{e}^{\prime}$. We then assume that the angular velocity and current components follow the near-rigid corotation approximations given by Eqs. (24) to (26) out to distance $\rho_{e}^{\prime}$, then breaking away to the behaviour defined by Eqs. (30) and (31) at larger distances, such that $j_{\| i}$ and hence $\Sigma_{P}^{*}$ reach the value specified at the outer boundary $\rho_{e}=\rho_{e B}\left(100 R_{J}\right.$ in the results presented here). Thus, introducing Eq. (25) into Eq. (30), the position $\rho_{e}^{\prime}$ where the break occurs is determined from the imposed boundary conditions by solving the equation

$$
\frac{2 \dot{M} \Omega_{J}}{\left|B_{z e}\left(\rho_{e}^{\prime}\right)\right|}=8 \pi \Sigma_{P}^{*}\left(\rho_{e B}\right) F_{e}\left(\rho_{e B}\right) \Omega_{J}\left(1-\left(\frac{\omega}{\Omega_{J}}\right)_{B}\right),
$$

where from Eq. (31)

$$
\begin{aligned}
& \left(\frac{\omega}{\Omega_{J}}\right)_{B} \approx\left(\frac{\rho_{e}^{\prime}}{\rho_{e B}}\right)^{2}\left(\frac{\omega}{\Omega_{J}}\right)^{\prime} \\
& +\frac{2}{\rho_{e B}^{2}\left|B_{z e}\left(\rho_{e}^{\prime}\right)\right|}\left(F_{e}\left(\rho_{e}^{\prime}\right)-F_{e}\left(\rho_{e B}\right)\right),
\end{aligned}
$$

and $\left(\omega / \Omega_{J}\right)^{\prime}$ is determined from Eq. (24) evaluated at $\rho_{e}^{\prime}$. With the value $\rho_{e}^{\prime}$ so determined, the approximate angular velocity profile is given by Eq. (31), the constant value of $I_{\rho}$ by Eq. (25) evaluated at $\rho_{e}^{\prime}$, and the conductivity profile from Eq. (30). The parallel current is determined by inversion of the expressions for the conductivity model given by Eq. (28). These approximations are shown by the dotted curves in Figs. 7 and 8, and are seen to agree quite well with the numerical solutions, particularly with the results for $S=10 \mathrm{mho}\left(\mu \mathrm{A} \mathrm{m}^{-2}\right)^{-1}$ shown in Fig. 8 .

\subsection{Solution dependence on $\dot{M}$}

The results shown above in Figs. 6 to 8 have all employed a fixed typical value of the iogenic plasma mass outflow rate $\dot{M}$ of $1000 \mathrm{~kg} \mathrm{~s}^{-1}$. Here we consider how the solutions depend on $\dot{M}$ for a given conductivity model. In Fig. 9 we show results in our standard format for $\dot{M}=1000$, 2000 , and $3000 \mathrm{~kg} \mathrm{~s}^{-1}$ (our particular interest in larger values becoming clearer in Sect. 5), for $S=10 \mathrm{mho}\left(\mu \mathrm{A} \mathrm{m}^{-2}\right)^{-1}$, and for the fixed boundary condition that $j_{\| i}=0.03 \mu \mathrm{A} \mathrm{m}^{-2}$ at $\rho_{e}=100 R_{J}$. In this case, however, three constant conductivity solutions are now shown in each plot for $\Sigma_{P}^{*}\left(j_{\| i}=0\right)=0.1 \mathrm{mho}$, corresponding to the three values of $\dot{M}$ (dashed lines as marked), and also three curves for the near-rigid corotation approximations given by Eqs. (24) to (26). However, for the case of the plasma angular velocity shown in Fig. 9a, although the departure of the angular velocity from rigid corotation is proportional to $\dot{M}$ in the innermost region, where $\Sigma_{P o}^{*}>S j_{\| i}$ in Eq. (28), over most of the radial range, where the opposite inequality applies, the nearrigid corotation approximation depends only on the slope $S$ and not on the mass outflow rate $\dot{M}$, i.e. if we put $\Sigma_{P}^{*} \simeq S j_{\| i}$ over most of the range, as given by Eq. (28), then substituting for $j_{\| i}$ from Eq. (26) into Eq. (24) yields

$$
\frac{\omega}{\Omega_{J}} \simeq 1-\frac{\rho_{e}\left|B_{z e}\right|^{2}}{4 S \Omega_{J} B_{J} F_{e}\left|\frac{d B_{z e}}{d \rho_{e}}\right|},
$$

such that the departure from rigid corotation is inversely proportional to $S$ (as can be seen in Figs. 7a and 8a), but is independent of $\dot{M}$. The angular velocity profiles in Fig. 9a thus follow essentially the same curves as each other in the inner region, before falling away from the near-rigid corotation approximation at larger distances. This latter distance decreases with increasing $\dot{M}$ (for fixed boundary condition) as can be seen in Fig. 9a, such that the plasma angular velocity at the outer boundary falls with increasing $\dot{M}$. Similarly, the current components and conductivity grow more rapidly in the inner region in proportion to $\dot{M}$, as shown in Figs. 9b$\mathrm{d}$ and as expected from Eqs. (25) and (26), while also falling away from this behaviour at smaller radial distances with increasing $\dot{M}$. The overall effect is that the main region of fieldaligned current flow into the current sheet moves inwards as $\dot{M}$ increases, for a given value of $j_{\| i}$ at the outer boundary. The value at which the total radial current plateaus in the outer region is also found to increase modestly with $\dot{M}$ under these condition, as can be seen in Fig. 9b.

\subsection{Results for a more realistic conductivity model}

The final point we wish to discuss in this section concerns the effect of the behaviour of the ionospheric Pedersen conductivity with the field-aligned current. It was assumed in the above calculations via Eq. (28) that the current increases essentially linearly with the current for all positive values of the latter (Fig. 5). This potentially results in very large values of the conductivity being obtained, as can be seen, for example, in Figs. 8d and 9d. It may be noted in the results presented in Sect. 3, however, that near-linear behaviour of the conductivity may only prevail for sufficiently small values of the field-aligned current density, with the conductivity tending to plateau or possibly even fall in value for larger values of $j_{\| i}$. We now investigate the effect of such conductivity behaviour by modifying the $S=10 \mathrm{mho}\left(\mu \mathrm{A} \mathrm{m}^{-2}\right)^{-1}$ model such that beyond a certain value of the field-aligned current density the conductivity increases much less rapidly than for small values of the current. Specifically, the model adopted is

$$
\begin{aligned}
& \Sigma_{P}^{*}\left(j_{\| i}\right)=\Sigma_{P o}^{*} \\
&+\frac{1}{2}\left\{S_{1}+\frac{S_{2}}{\left[1+\left(\frac{j_{\| i}}{j_{\| i}^{* *}}\right)^{n}\right]^{\frac{1}{n}}}\right\}\left(\sqrt{j_{\| i}^{2}+j_{\| i}^{* 2}}+j_{\| i}\right),
\end{aligned}
$$

which is such that $\Sigma_{P}^{*} \simeq \Sigma_{P o}^{*}$ for $j_{\| i}$ negative, varies as $\Sigma_{P}^{*} \simeq \Sigma_{P o}^{*}+\left(S_{1}+S_{2}\right) j_{\| i}$ for positive $j_{\| i}$ less than $j_{\| i}^{* *}$, and then as $\Sigma_{P}^{*} \simeq\left(\Sigma_{P o}^{*}+S_{2} j_{\| i}^{* *}\right)+S_{1} j_{\| i}$ for $j_{\| i}$ greater than $j_{\| i}^{* *}$. 


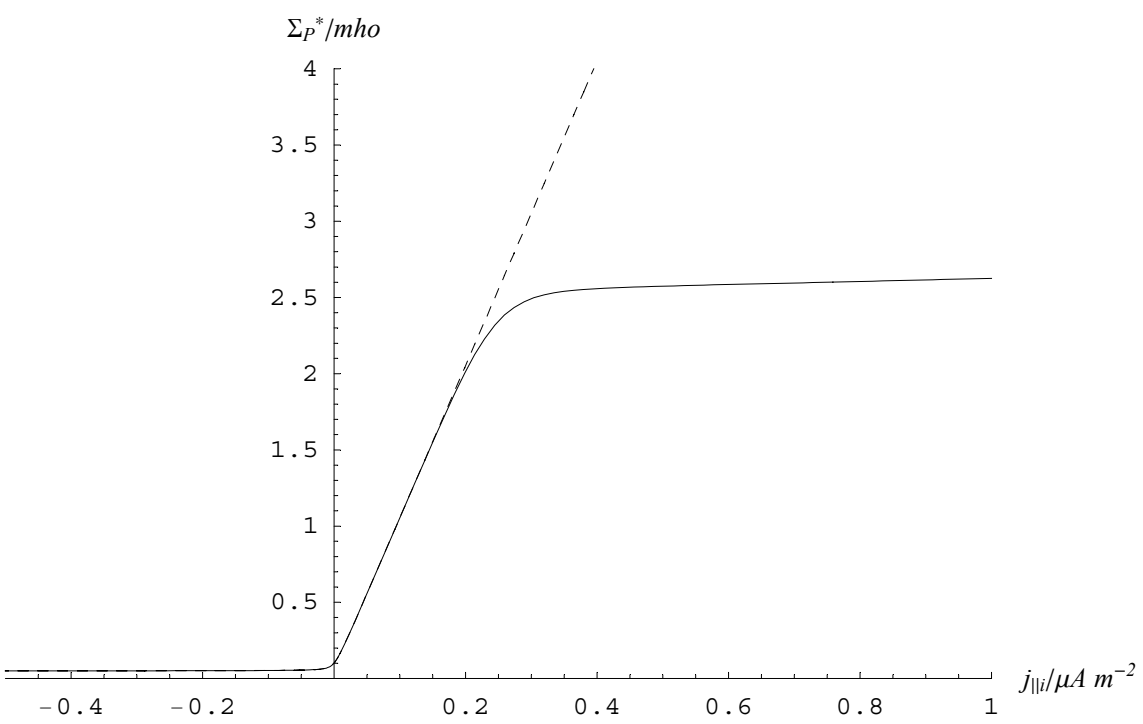

Fig. 10. Plot of the effective height-integrated Pedersen conductivity $\Sigma_{P}^{*}$ in mho versus ionospheric field-aligned current density $j_{\| i}\left(\mu \mathrm{A} \mathrm{m}^{-2}\right)$ using the revised conductivity model given by Eq. (35) (solid line). This is compared with the near-linear conductivity model given by Eq. (28) with slope $S=10 \mathrm{mho}\left(\mu \mathrm{A} \mathrm{m}^{-2}\right)^{-1}$ (dashed line).

Here we have taken $\Sigma_{P o}^{*}=0.05 \mathrm{mho}$ and $j_{\| i}=0.01 \mu \mathrm{A} \mathrm{m}^{-2}$, as before, together with $S_{1}=0.1 \mathrm{mho}\left(\mu \mathrm{A} \mathrm{m}^{-2}\right)^{-1}$, $S_{2}=9.9 \mathrm{mho}\left(\mu \mathrm{A} \mathrm{m}^{-2}\right)^{-1}, \quad j_{\| i}^{* *}=0.25 \mu \mathrm{A} \mathrm{m}^{-2}$ and $n=8$. This function is shown in Fig. 10 (solid line), together with the $S=10 \mathrm{mho}\left(\mu \mathrm{A} \mathrm{m}^{-2}\right)^{-1}$ model given by Eq. (28) (dashed line). It can be seen that the two models are essentially identical for small positive and all negative values of $j_{\| i}$, with the conductivity increasing at the rate of $\sim 10 \mathrm{mho}\left(\mu \mathrm{A} \mathrm{m}^{-2}\right)^{-1}$ for positive $j_{\| i}$ less than $0.25 \mu \mathrm{A} \mathrm{m}^{-2}$. Above this value of the current, however, the conductivity curve rapidly flattens to increase at the much reduced rate of $0.1 \mathrm{mho}\left(\mu \mathrm{A} \mathrm{m}^{-2}\right)^{-1}$.

Results using this conductivity model are shown in Fig. 11 in the same format as Figs. 6 to 9. Here we compare the solution obtained with the above model with that obtained with the $S=10$ mho $\left(\mu \mathrm{A} \mathrm{m}^{-2}\right)^{-1}$ model of Eq. (28), for the same value of $\dot{M}=1000 \mathrm{~kg} \mathrm{~s}^{-1}$, and with the same boundary condition $j_{\| i}=0.03 \mu \mathrm{A} \mathrm{m}^{-2}$ at $\rho_{e}=100 R_{J}$. We note that the dashed line showing the constant conductivity solution for $\Sigma_{P}^{*}=\Sigma_{P}^{*}\left(j_{\| i}=0\right)=0.1$ mho is the same for both models. The current profiles of the near-rigid corotation approximations Eqs. (25) and (26), shown by the dot-dashed lines, are also the same for the two calculations, being dependent only on $\dot{M}$ and the equatorial magnetic field model. However, the angular velocity profiles in the latter approximation given by Eq. (24) are in general different, being dependent on the conductivity model. As can be seen in Fig. 11a, the two profiles are very similar to each other in the inner region, where the field-aligned current given by Eq. (26) lies below $\sim 0.25 \mu \mathrm{A} \mathrm{m}^{-2}$, such that the model $\Sigma_{P}^{*}$ values are closely similar to each other. However, the field-aligned current approximation exceeds this value at and beyond $\rho_{e} \sim 25 R_{J}$, such that in the revised model the conductivities then fall significantly below those given by the near-linear model with
$S=10 \mathrm{mho}\left(\mu \mathrm{A} \mathrm{m}^{-2}\right)^{-1}$ (Fig. 10), and with it, the departure of the plasma from rigid corotation given by Eq. (24) also significantly increases. It can be seen in Fig. 11a that in the inner region the numerically integrated angular velocity profiles follow their respective approximations, closely in the case of the $S=10 \mathrm{mho}\left(\mu \mathrm{A} \mathrm{m}^{-2}\right)^{-1}$ near-linear model out to $\sim 40 R_{J}$, and approximately in the case of the revised conductivity model given by Eq. (35) out to $50 R_{J}$, before falling more rapidly in the outer region to values which are quite similar at the outer boundary. The current profiles shown in Figs. $11 \mathrm{~b}$ and $11 \mathrm{c}$ are also similar to each other. The parallel current for the revised conductivity model in Fig. 11c lies modestly below that of the $S=10 \mathrm{mho}\left(\mu \mathrm{A} \mathrm{m}^{-2}\right)^{-1}$ model beyond $\sim 20 R_{J}$, while peaking at a larger value at a larger distance, before falling precipitately to small values beyond $\sim 40 R_{J}$. The total radial current profiles shown in Fig. 11b behave in a corresponding manner, with closely similar values being achieved at the outer boundary at $\rho_{e}=100 R_{J}$. Turning now to the conductivity profile shown in Fig. 11d, it can be seen that the effect of the revised conductivity model is to truncate the increase in conductivity in the central regions to reach a peak of only $\sim 3$ mho, compared with $\sim 20$ mho for the near-linear model. Nevertheless, the elevation of the conductivity in the revised model is still sufficient to maintain the plasma angular velocity and the current components close to the values given by the near-rigid corotation approximation out to significant distances. The main distinction between the two models is that the angular velocity is more significantly depressed from rigid corotation in the revised model, such as to maintain similar values of the currents. 
$\omega / \Omega_{J}$
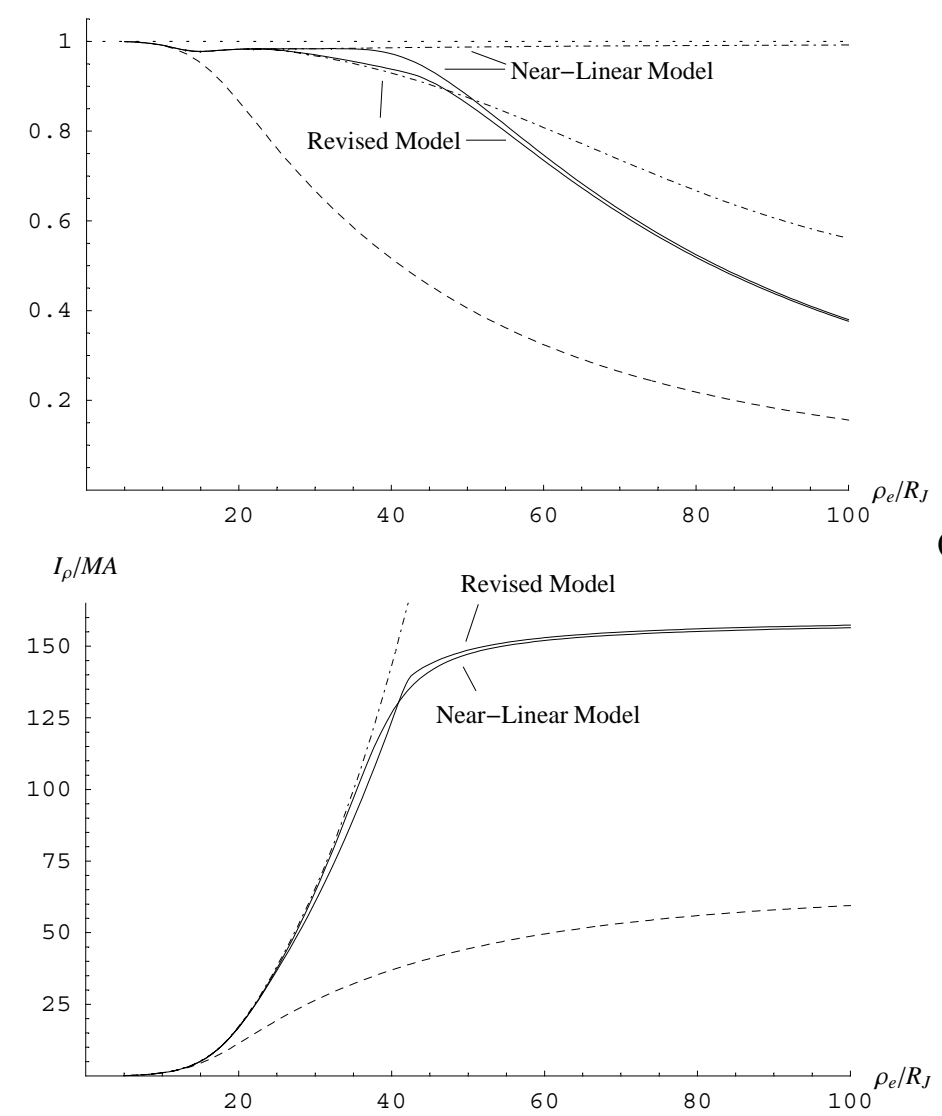

$j_{\| i} / \mu A m^{-2}$

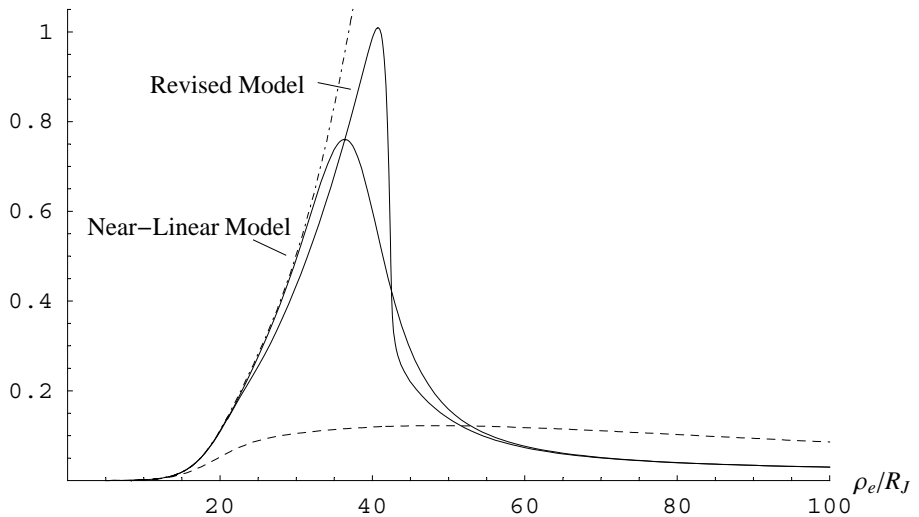

$\Sigma_{P}{ }^{*} / m h o$

(d)

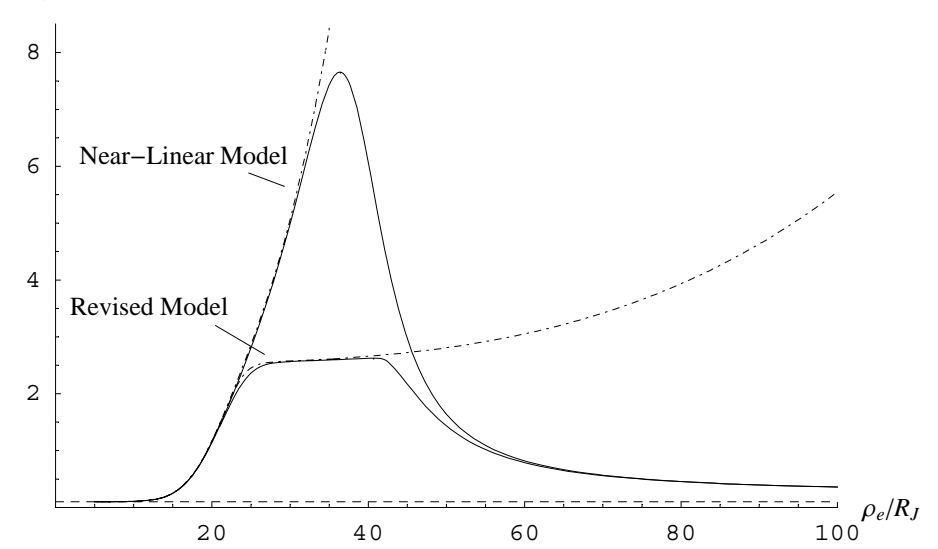

(a)

(b)
Fig. 11. Plots of the magnetosphereionosphere coupling current system parameters for the near-linear conductivity model (Eq. 28) with slope $S=10 \mathrm{mho}\left(\mu \mathrm{A} \mathrm{m}^{-2}\right)^{-1}, \quad$ and the revised conductivity model (Eq. 35) (solid lines as labelled), for constant conductivity $\quad \Sigma_{P}^{*}\left(j_{\| i}=0\right)=0.1$ mho (dashed lines), and for the near-rigid corotation approximations given by Eqs. (24)-(26) (dot-dashed lines). The solutions are tracked to $13 R_{J}$ and $21 R_{J}$ for the near-linear and revised models, respectively, inside which they are completed by the near-rigid approximation. All parameters are plotted versus jovicentric equatorial radial distance $\rho_{e}$. Plot (a) shows the plasma angular velocity $\omega$ normalised to the planet's angular velocity, plot (b) shows the azimuth-integrated equatorial radial current $I_{\rho}$ in MA, plot (c) shows the field-aligned current density at the feet of the field lines $j_{\| i}$ in $\mu \mathrm{A} \mathrm{m}^{-2}$, while plot (d) shows the effective height-integrated Pedersen conductivity $\Sigma_{P}^{*}$ in mho. 


\section{Solutions appropriate to Jovian conditions}

In this section we examine solutions for the angular velocity and current components which are appropriate to conditions in the Jovian magnetosphere, and consider how well they fit to available observations. Specifically, we derive solutions based on the empirical conductivity model given by Eq. (23), shown by the dashed line in Fig. 4, and we also choose boundary conditions that yield solutions appropriate to the Jovian system.

\subsection{Observed radial current profile}

The Jovian observations to which we refer are the radial profiles of the radial equatorial current $i_{\rho}$ derived from Galileo magnetometer data by Khurana (2001). As discussed in the Introduction, the radial current is associated with the bending of the magnetic field lines out of meridian planes, and hence, with the appearance of a $B_{\varphi}$ field which reverses in sense about the centre of the equatorial current sheet, as shown in Fig. 1. Applying Ampère's law to a loop passing through the current sheet and closing in the region outside, we find

$i_{\rho}=\mp \frac{2 \Delta B_{\varphi}}{\mu_{o}}$

where $\Delta B_{\varphi}$ is the azimuthal field outside the current sheet (the planetary azimuthal field is negligible at middle magnetosphere distances). The upper sign applies to observations north of the current sheet, while the lower sign applies to observations south of the sheet, and we assume north-south symmetry of the field line bending. In Fig. $12 \mathrm{~b}$ we show the radial profile of the radial current derived from Galileo data, kindly provided at increased $5 R_{J}$ radial resolution by K. K. Khurana (personal communication, 2002). This has been derived using Eq. (36) from magnetic data outside the current sheet, within a 3-h local time sector centred on midnight. Data from this sector have been chosen since it is less likely to contain systematic contributions from other effects that produce magnetospheric $\Delta B_{\varphi}$, such as field line bending associated with the day-night asymmetry of the magnetospheric cavity due to the dynamic pressure of the solar wind. The resulting values of $i_{\rho}$ derived from Eq. (36) have then been multiplied by $2 \pi \rho_{e}$, in order to represent the total radial current integrated in azimuth, as seems appropriate as a first approximation (see also Khurana, 2001). It can be seen that the total radial current inferred from this data increases rapidly in the inner region, between $\sim 15$ and $25 R_{J}$, before plateauing at a value of $\sim 100 \mathrm{MA}$ at distances beyond, out to $\sim 100 R_{J}$. As noted previously by Khurana (2001), these results imply that the upward-directed field-aligned currents which feed the current sheet are concentrated in the inner region, centred near $\sim 20 R_{J}$, with the upward field-aligned current density in the ionsphere derived from the slope of the curve in this region being typically $\sim 0.2-0.3 \mu \mathrm{A} \mathrm{m}^{-2}$.

\subsection{Comparison with constant conductivity model results}

The dashed lines in Fig. 12b show solution curves for $I_{\rho}$ derived for the typical value of $\dot{M}=1000 \mathrm{~kg} \mathrm{~s}^{-1}$ and various constant values of the effective Pedersen conductivity, 0.1, 0.2 and $0.3 \mathrm{mho}$, as marked, in the range used in previously published studies. These are plotted together with the limiting value of the current given by Eq. (25), shown by the dotdashed line. The other panels of the figure show the other parameters of these solutions, specifically $\left(\omega / \Omega_{J}\right)$ in Fig. 12a and $j_{\| i}$ in Fig. 12c. It can be seen that although these constant conductivity solutions produce $I_{\rho}$ profiles which are not unadjacent to that derived from the Galileo data, they also do not provide a good "fit" for any reasonable values of the system parameters $\Sigma_{P}^{*}$ and $\dot{M}$. The "best fit" solutions (e.g. that for $\dot{M}=1000 \mathrm{~kg} \mathrm{~s}^{-1}$ and $\Sigma_{P}^{*}=0.2$ to 0.3 mho in Fig. 12b) tend to rise too gradually in the inner region and to overshoot at larger distances, associated with the broadly-distributed profile of the upward field-aligned current shown in Fig. 12c.

In Fig. 13 we also show for future reference the plasma angular velocity and current components for these constant conductivity solutions mapped along field lines into the ionosphere, using Eq. (4). In this format the plots extend from a co-latitude of $\sim 15.7^{\circ}$, mapping to $100 R_{J}$ in the equatorial plane, to $19^{\circ}$, mapping to $\sim 12.1 R_{J}$. In Fig. 13a we show the angular velocity profiles (dashed), together with the approximate forms (dot-dashed), while in Figs. 13b and $13 \mathrm{c}$ we show the total height-integrated Pedersen current integrated in azimuth, equal to half the conjugate equatorial current $I_{\rho}$, together with the similarly halved Galileo data, and the field-aligned current density, respectively, in a similar format. These theoretical plots are entirely representative of previously-published results, showing, for example, the field-aligned current density peaking at a few tenths of a $\mu \mathrm{A} \mathrm{m}^{-2}$ near the poleward boundary of the ionospheric region mapping to the middle magnetosphere, and falling to small values over a latitudinal scale of $\sim 1.5^{\circ}$ FWHM (i.e. $\sim 2000 \mathrm{~km}$ ) in the ionosphere. In Figs. 13d and 13e we also show estimates of the auroral accelerating voltage and the precipitating auroral electron energy flux, derived from Eqs. (15) and (16) (using the full formulae rather than the approximate forms), with magnetospheric electron parameters $N=0.01 \mathrm{~cm}^{-3}$ and $W_{t h}=2.5 \mathrm{keV}$, as employed in previous papers and above in Sect. 3. These show that accelerating voltages of several tens of $\mathrm{kV}$ are anticipated, together with precipitating energy fluxes of a few tens of $\mathrm{mW} \mathrm{m}^{-2}$, in line with previous results (e.g. Cowley and Bunce, 2001; Cowley et al., 2002, 2003). We recall that at $\sim 20 \%$ conversion efficiency, an energy flux of $10 \mathrm{~mW} \mathrm{~m}^{-2}$ corresponds to a UV luminosity of $\sim 100 \mathrm{kR}$, such that typical luminosities are expected to be of this order, as observed.

\subsection{Empirical conductivity model}

We now turn to the results for varying conductivity, and first discuss the conductivity model to be employed. As indicated above, this is based on the empirical form Eq. (23) 

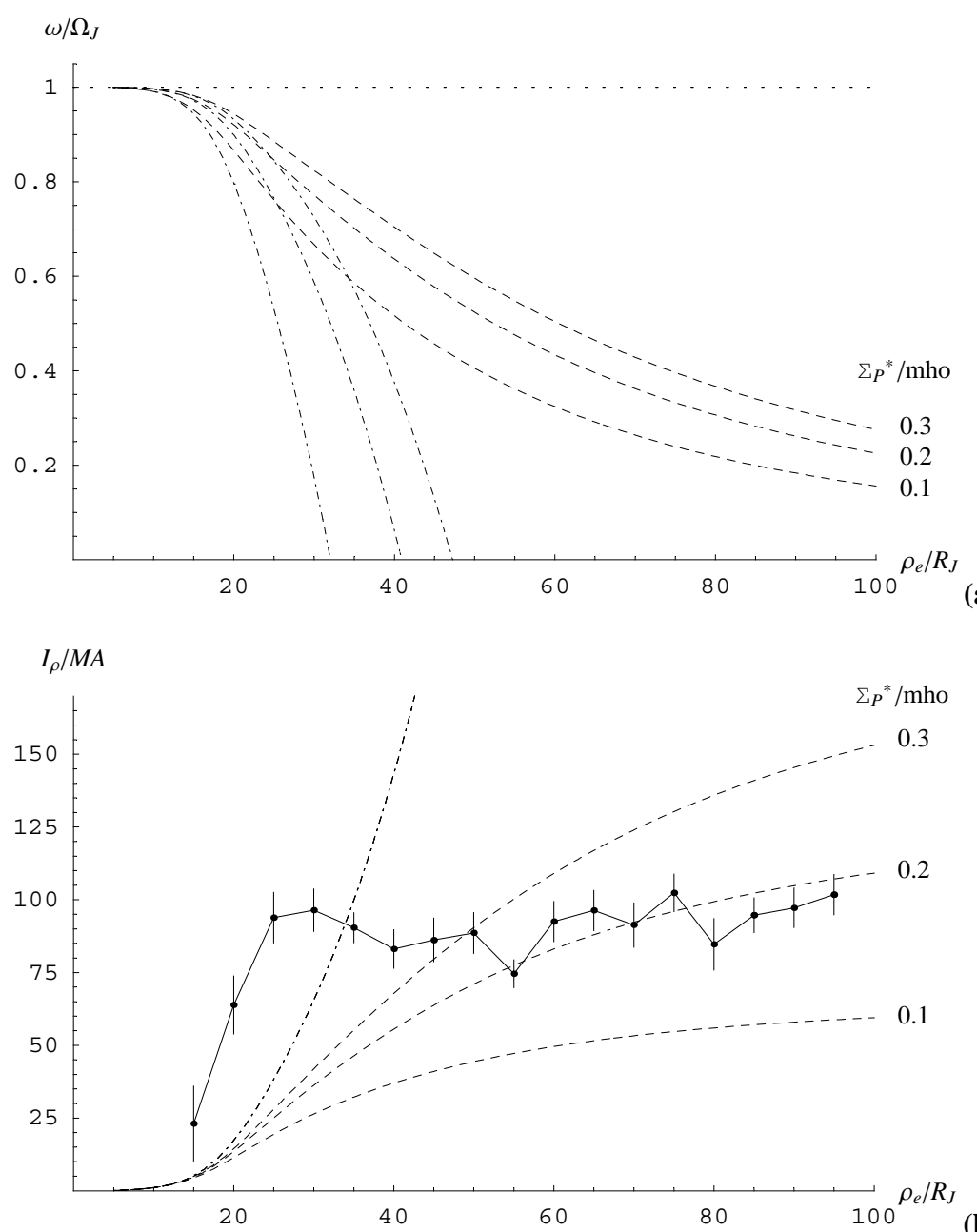

(a)

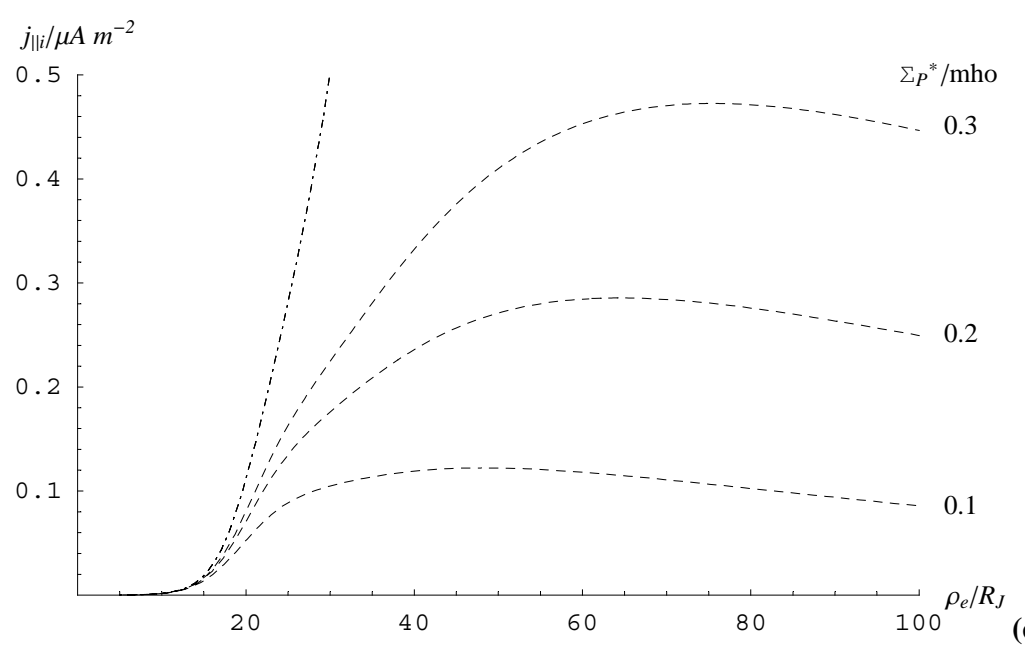

Fig. 12. Plots of the magnetosphere-ionosphere coupling current system parameters using $\dot{M}=1000 \mathrm{~kg} \mathrm{~s}^{-1}$ and constant effective heightintegrated Pedersen conductivities of $0.1,0.2$ and 0.3 mho (dashed lines as labelled), together with the near-rigid corotation approximations given by Eqs. (24)-(26) (dot-dashed lines). All parameters are plotted versus jovicentric equatorial radial distance $\rho_{e}$. Plot (a) shows the plasma angular velocity $\omega$ normalised to the planet's angular velocity $\Omega_{J}$, plot (b) shows the azimuth-integrated equatorial radial current $I_{\rho}$ in MA, while plot (c) shows the field-aligned current density at the feet of the field lines $j_{\| i}$ in $\mu \mathrm{A} \mathrm{m}^{-2}$. In plot (b) the theoretical curves are compared with the radial profile of the radial current derived from Galileo data in the midnight sector, as described in the text. 

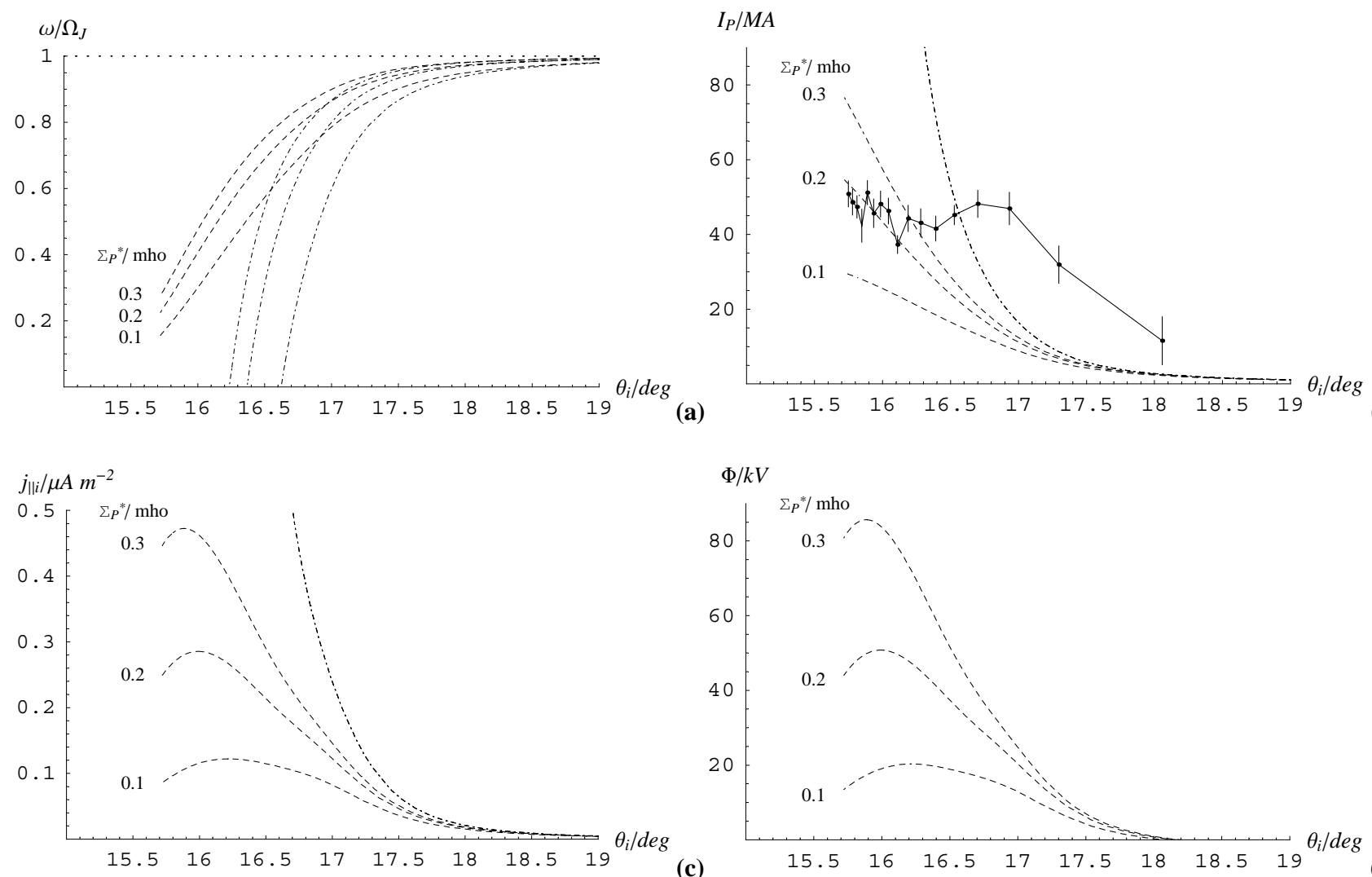

(d)

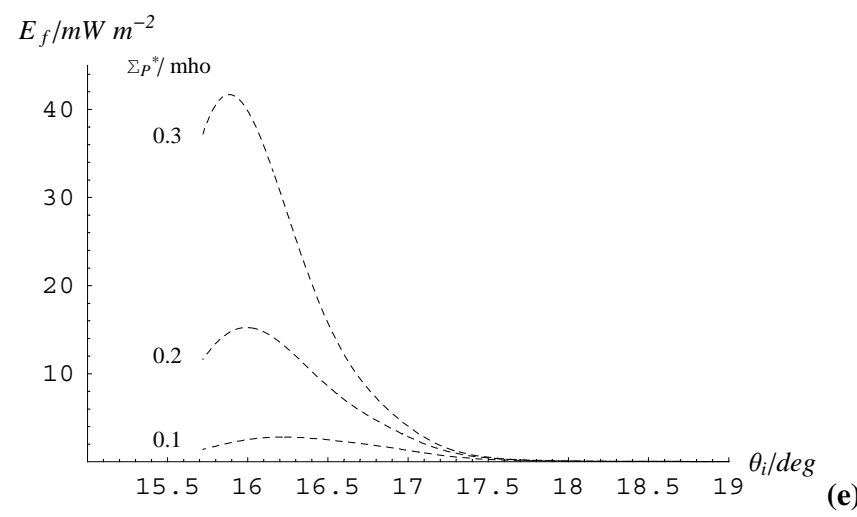

Fig. 13. Panels (a), (b) and (c) show the magnetosphere-ionosphere coupling current system parameters for constant conductivity and the Galileo data shown previously in Fig. 12a-c, but now projected along model magnetic field lines into the Jovian ionosphere and plotted versus dipole co-latitude $\theta_{i}$, using Eq. (4). In panel (b) the equatorial radial current $I_{\rho}$ has also been divided by a factor of two to show the azimuth-integrated Pedersen current flowing in one ionosphere, $I_{P}$. Plot (d) then shows the minimum accelerating voltage $\Phi$ in $\mathrm{kV}$ required to drive the field-aligned current obtained from the exact form of Eq. (15). Plot (e) similarly shows the precipitating energy flux $E_{f}$ in $\mathrm{mW} \mathrm{m}^{-2}$ obtained from the exact form of Eq. (16).

derived above in Sect. 3 from the modelling results presented by Millward et al. (2002). However, the quantity estimated above is the true height-integrated Pedersen conductivity $\Sigma_{P}$, while the value required here is the effective value $\Sigma_{P}^{*}$, equal to $(1-k) \Sigma_{P}$, reduced from the true value by atmospheric "slippage" discussed in Sect. 2.2 above. The appropriate value of $k$ is not conclusively known at the present time, but recent modelling results (G. H. Millward, personal communication, 2003) indicate that $k \approx 0.5$ under circumstances appropriate to those discussed in this paper. Here we will therefore adopt this value, such that we will take the factor $(1-k)=0.5$ in Eq. (6). Noting that the empirical form Eq. (23) goes to zero for $j_{\| i}=0$, we will also add a small constant conductivity $\Sigma_{P o}^{*}$. As found in Sect. 4, the value of $\Sigma_{P o}^{*}$ governs the nature of the angular velocity profile at small distances where $j_{\| i}$ becomes small. This value of 


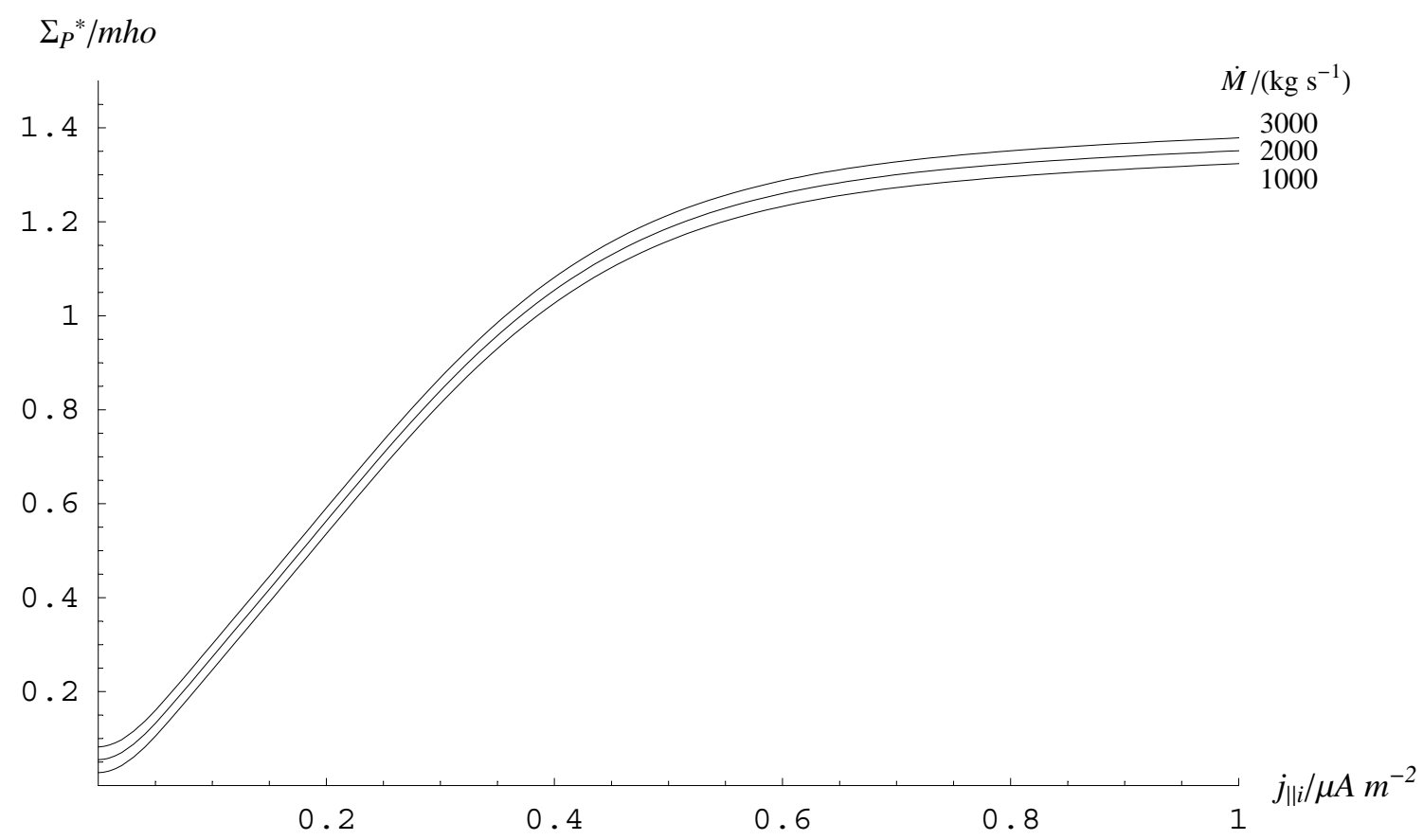

Fig. 14. Plot of the effective height-integrated Pedersen conductivity $\Sigma_{P}^{*}$ in mho versus ionospheric field-aligned current density $j_{\| i}\left(\mu \mathrm{A} \mathrm{m}^{-2}\right)$ using the empirical conductivity model given by Eq. (37).

$\Sigma_{P o}^{*}$ has thus been determined from the results presented by Hill (1980), who examined Voyager-1 angular velocity data in the inner region (from $\sim 12 R_{J}$ to $\sim 20 R_{J}$ ) and compared the data with constant conductivity solutions for a dipole field model (which is reasonably realistic in the inner region). The theoretical angular velocity profiles depend on the quotient $\Sigma_{P}^{*} / \dot{M}$, the results obtained by Hill (1980) indicating that $\Sigma_{P}^{*} / \dot{M} \approx 2.75 \times 10^{-5} \mathrm{mho}\left(\mathrm{kg} \mathrm{s}^{-1}\right)$. Although it is not a major point of our study, we have therefore employed here $\Sigma_{P o}^{*}=0.0275 \times \dot{M}\left(1000 \mathrm{~kg} \mathrm{~s}^{-1}\right)$ mho, such that the calculated angular velocity profile perforce asymptotes to the form determined by Hill (1980) in the inner region (and by which we do not wish to imply that the "background" ionospheric conductivity is somehow physically determined by the mass outflow rate from Io). In summary, therefore, here we employ the following empirical conductivity model

$$
\begin{aligned}
\Sigma_{P}^{*} & =0.0275 \dot{M}+0.08 j_{\| i}+\left\{1.225\left[\frac{\left(j_{\| i} / 0.075\right)^{2}}{1+\left(j_{\| i} / 0.075\right)^{2}}\right]\right. \\
& \left.\times \frac{1}{1+\exp \left[-\left(j_{\| i}-0.22\right) / 0.12\right]}\right\},
\end{aligned}
$$

where $\dot{M}$ is in units of $1000 \mathrm{~kg} \mathrm{~s}^{-1}$, and $j_{\| i}$ is in $\mu \mathrm{Am}^{-2}$. In Fig. 14 we show $\Sigma_{P}^{*}$ versus $j_{\| i}$ for $\dot{M}=1000,2000$ and $3000 \mathrm{~kg} \mathrm{~s}^{-1}$, as employed below.

\subsection{Results using the empirical conductivity model}

Results using the empirical conductivity model given by Eq. (37) are shown in our standard format in Fig. 15. The solid lines show three numerically computed solutions for
$\dot{M}=1000,2000$ and $3000 \mathrm{~kg} \mathrm{~s}^{-1}$, each employing differing values of $j_{\| i}$ at the boundary at $\rho_{e}=100 R_{J}$, such that the total current $I_{\rho}$ at $100 R_{J}$ is equal to $100 \mathrm{MA}$, in approximate agreement with the values obtained at large distance from the Galileo data. The values of $j_{\| i}\left(100 R_{J}\right)$ employed are $\sim 0.264, \sim 0.171$ and $\sim 0.133 \mu \mathrm{A} \mathrm{m}^{-2}$ for $\dot{M}=1000,2000$ and $3000 \mathrm{~kg} \mathrm{~s}^{-1}$, respectively. The dot-dashed lines show the corresponding near-rigid corotation approximations given by Eqs. (24) to (26), while the dashed lines show the constant conductivity solutions with $\Sigma_{P}^{*}=\Sigma_{P}^{*}\left(j_{\| i}=0\right)$. There is only one constant conductivity solution for the angular velocity due to the above choice of the form of $\Sigma_{P o}^{*}$.

We begin by comparing the $I_{\rho}$ profiles shown in Fig. 15b with the values derived from the Galileo data. It can be seen that the shape of the theoretical curves agree reasonably well with the Galileo data, consisting of a sharp rise in the inner region, followed by an extended region of almost constant values. However, it can be seen that the initial rise in the current occurs at too large a radial distance for $\dot{M}=1000 \mathrm{~kg} \mathrm{~s}^{-1}$, and approaches more satisfactory agreement as $\dot{M}$ increases to 2000 and $3000 \mathrm{~kg} \mathrm{~s}^{-1}$, thus explaining our interest in larger values of $\dot{M}$ indicated above. However, even the results for $3000 \mathrm{~kg} \mathrm{~s}^{-1}$ do not quite reach the mean of the Galileo data in the innermost region, while the observational estimates outlined in the Introduction suggest an upper limit of the mass transport rate of $\sim 2000 \mathrm{~kg} \mathrm{~s}^{-1}$, with smaller values of $\sim 1000 \mathrm{~kg} \mathrm{~s}^{-1}$ being more typical. The implication may therefore be that the mean $\left|B_{z e}\right|$ in the model employed here is too large (see, e.g. Eq. 25) in the critical upward current region, a possibility that needs to be carefully examined in future study. 

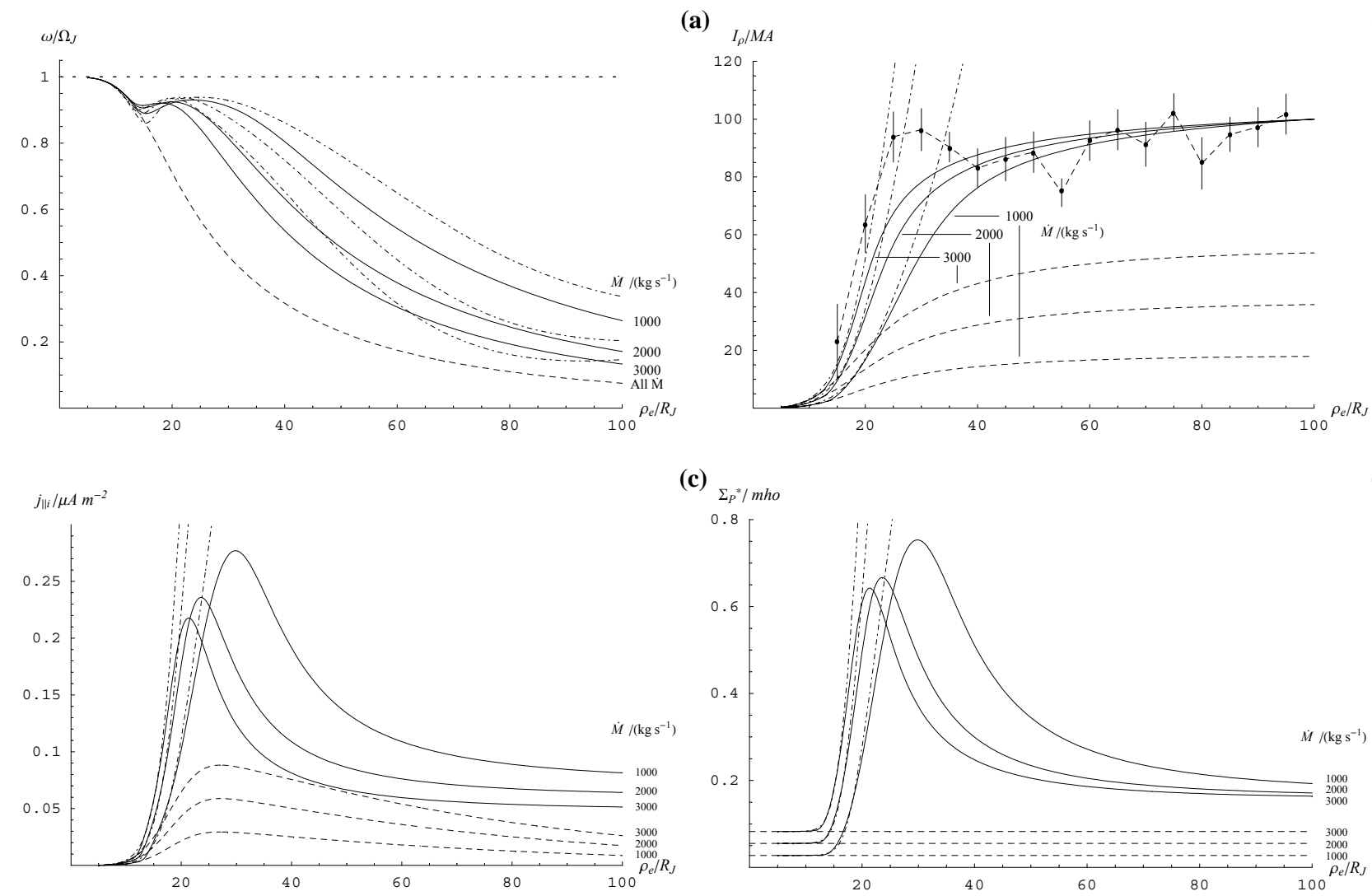

(c)

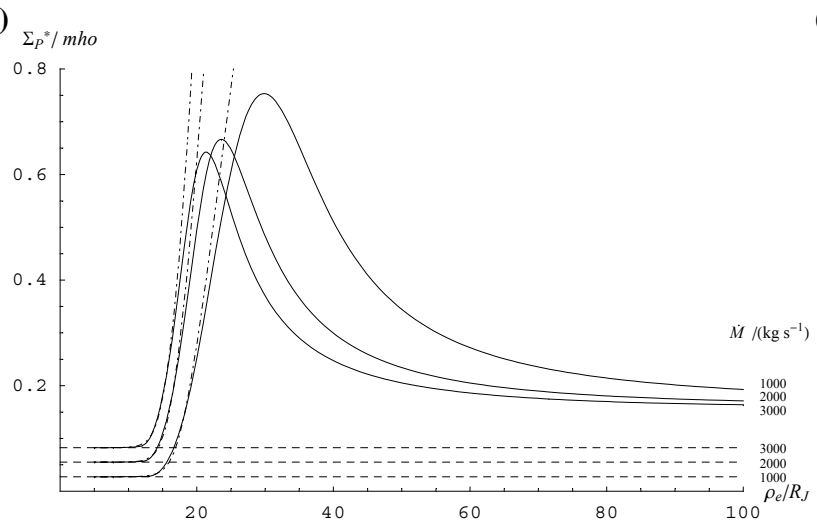

(d)

Fig. 15. Plots of the magnetosphere-ionosphere coupling current system parameters obtained using the empirical conductivity model (Eq. 37) (solid lines), constant conductivity $\Sigma_{P}^{*}=\Sigma_{P}^{*}\left(j_{\| i}=0\right)$ (dashed lines), and the near-rigid corotation approximations given by Eqs. (24)-(26) (dot-dashed lines), for three values of the iogenic plasma mass outflow rate $\dot{M}=1000,2000$ and $3000 \mathrm{~kg} \mathrm{~s}^{-1}$ (as indicated). The empirical conductivity model solutions have the boundary condition on $j_{\| i}$ set such that the value of the azimuth-integrated equatorial radial current in each case is equal to $100 \mathrm{MA}$. The solutions are tracked numerically to $12 R_{J}$, inside which they are completed by the "first-iteration" approximation of Sect. 4.2. All parameters are plotted versus jovicentric equatorial radial distance $\rho_{e}$. Plot (a) shows the plasma angular velocity $\omega$ normalised to the planet's angular velocity $\Omega_{J}$, plot (b) shows the azimuth-integrated equatorial radial current $I_{\rho}$ in MA, together with the radial profile of the radial current derived from Galileo $B_{\varphi}$ data provided by Khurana, plot (c) shows the field-aligned current density at the feet of the field lines $j_{\| i}$ in $\mu \mathrm{A} \mathrm{m}^{-2}$, while plot (d) shows the effective height-integrated Pedersen conductivity $\Sigma_{P}^{*}$ in mho.

Turning now to the results for the field-aligned current shown in Fig. $15 \mathrm{c}$, we see that the $j_{\| i}$ profiles all strongly peak in the inner region with similar peak values of between $\sim 0.22$ and $\sim 0.28 \mu \mathrm{A} \mathrm{m}^{-2}$ for $\dot{M}=3000 \mathrm{~kg} \mathrm{~s}^{-1}$ and $1000 \mathrm{~kg} \mathrm{~s}^{-1}$, respectively, before falling rapidly at larger distances. However, as may be expected from the results discussed in Fig. 15b, the position of the peak moves in towards the planet as $\dot{M}$ increases, from $\rho_{e} \approx 30 R_{J}$ when $\dot{M}=1000 \mathrm{~kg} \mathrm{~s}^{-1}$, to $\rho_{e} \approx 20 R_{J}$ when $\dot{M}=3000 \mathrm{~kg} \mathrm{~s}^{-1}$. The Pedersen conductivity curves in Fig. 15d show a corresponding behaviour, peaking in the inner region at $\sim 0.7$ mho.

Finally, the corresponding angular velocity curves are shown in Fig. 15a. These show similar initial decreases to each other in the innermost region (as guaranteed by the choice of $\Sigma_{P o}^{*}$ ), before rising again outside $\sim 15 R_{J}$, in line with the "near-rigid" corotation approximation Eq. (24), shown by the dot-dashed lines, and then falling more gradually at larger distances to values at the outer boundary which decrease with increasing $\dot{M}$. These results show how it is possible to deduce small values of $\Sigma_{P}^{*}$ (for typical values of $\dot{M}$ ) from plasma angular velocity profiles in the inner region, as found by Hill (1980), while values at larger distances are much higher than would be anticipated on this basis, as found, for example, by Kane et al. (1995) and Krupp et al. (2001).

In Fig. 16 we show these parameters mapped along the field lines into the ionosphere, together with auroral parameters derived from Eqs. (15) and (16), as in Figs. 13d and $13 \mathrm{e}$. Figure 16a shows that the elevated conductivity conditions produced by the auroral precipitation maintains nearrigid corotation conditions up to dipole co-latitudes of $\sim 17^{\circ}$, before falling rapidly to smaller values over $\sim 1^{\circ}$ latitude in the poleward region. The total height-integrated Pedersen current curves $\left(I_{P}=I_{\rho} / 2\right)$ shown in Fig. 16b exhibit related behaviour, with values elevated between $\sim 16.5^{\circ}$ and $17.5^{\circ}$ by the precipitation-enhanced Pedersen conductivity. 

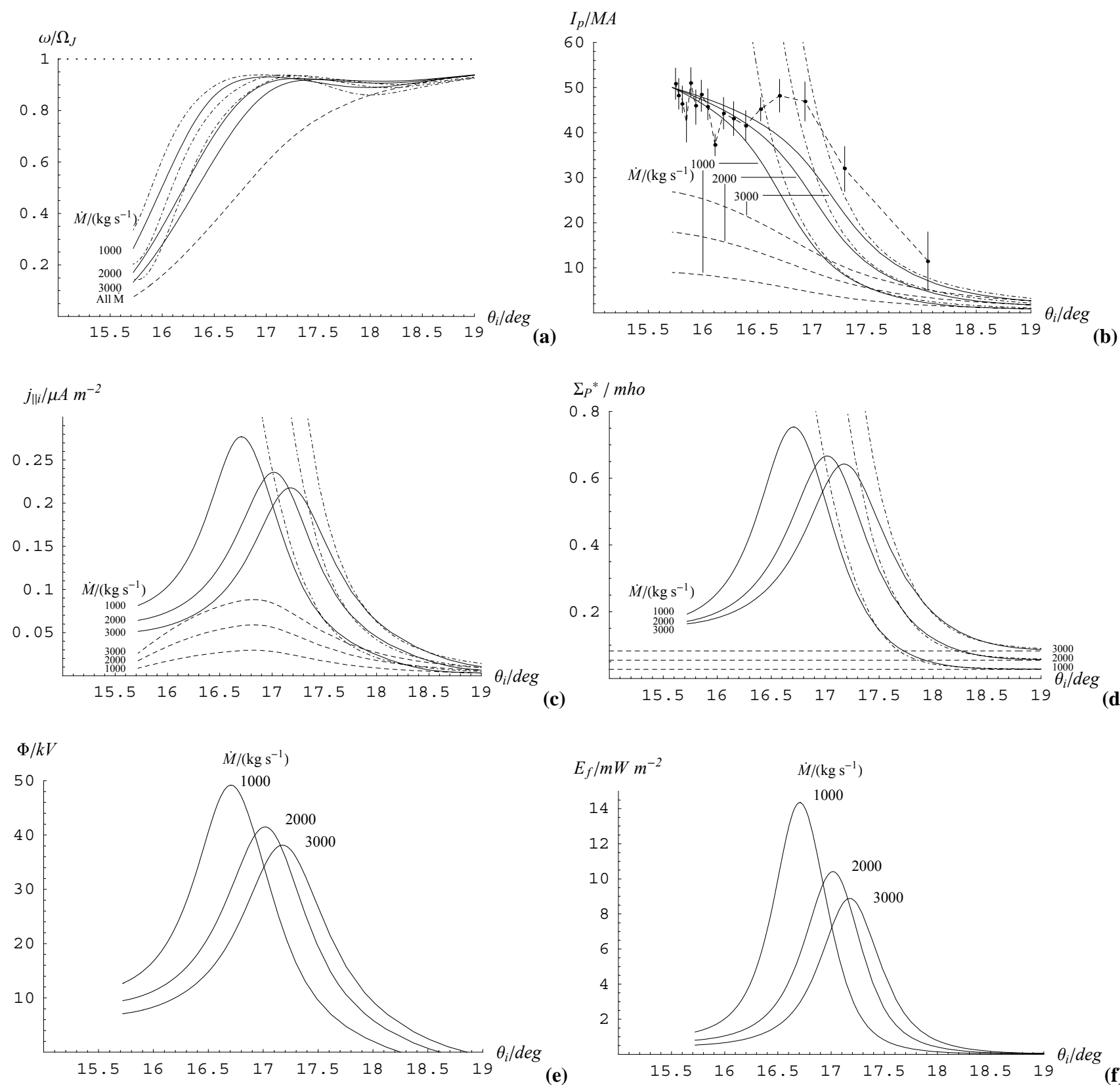

Fig. 16. Plots of the solutions shown in Fig. 15 are shown projected along the field lines into the ionosphere, using the same line style format. All parameters are plotted versus dipole co-latitude $\theta_{i}$. Plot (a) shows the plasma angular velocity $\omega$ normalised to the planet's angular velocity $\Omega_{J}$, plot (b) shows the azimuth-integrated ionospheric Pedersen current $I_{P}$ in MA, together with the Pedersen current derived from Galileo data, plot (c) shows the field-aligned current density $j_{\| i}$ in $\mu \mathrm{A} \mathrm{m}^{-2}$, plot (d) shows the effective height-integrated Pedersen conductivity in mho, plot (e) shows the minimum accelerating voltage $\Phi$ in $\mathrm{kV}$ required to drive the field-aligned current, obtained from the exact form of Eq. (15), while plot (f) shows the precipitating energy flux $E_{f}$ in $\mathrm{mW} \mathrm{m}^{-2}$ obtained from the exact form of Eq. (16).

The field-aligned current profiles shown in Fig. 16c show similar curves peaking between $\sim 0.22$ and $\sim 0.28 \mu \mathrm{A} \mathrm{m}^{-2}$ for $\dot{M}=3000 \mathrm{~kg} \mathrm{~s}^{-1}$ and $1000 \mathrm{~kg} \mathrm{~s}^{-1}$, respectively, at colatitudes moving equatorward from $16.7^{\circ}$ to $17.2^{\circ}$ as $\dot{M}$ increases from $1000 \mathrm{~kg} \mathrm{~s}^{-1}$ to $3000 \mathrm{~kg} \mathrm{~s}^{-1}$, in corresponding behaviour with the $I_{P}$ curves. Comparison with the profiles for constant conductivity shown in Fig. 13c show that the current distributions are now significantly narrower, with a width (FWHM) of $\sim 1^{\circ}(\sim 1300 \mathrm{~km})$. The conductivity profiles in Fig. 16d show corresponding behaviour, peaking at $\sim 0.7$ mho at $\sim 17^{\circ}$. This is significantly larger than both the background conductivity and the assumed constant conductivity values which have been taken in previous papers. The estimated accelerating voltages and precipitating energy 


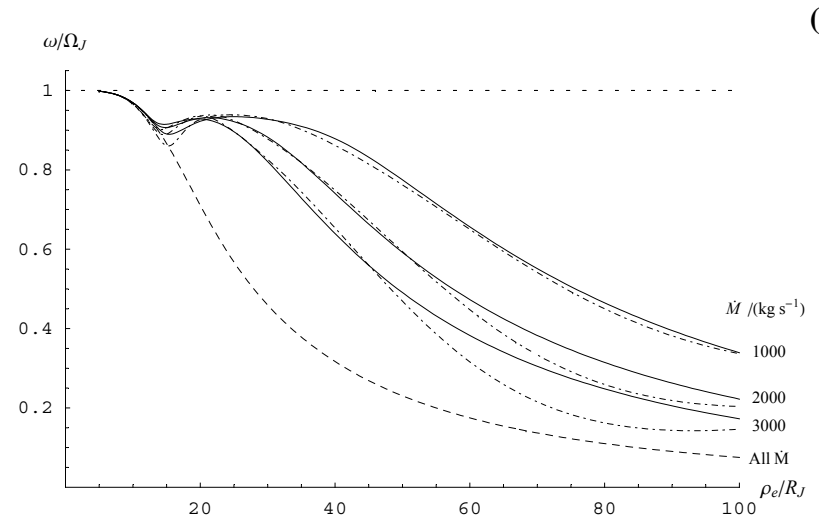

(a)

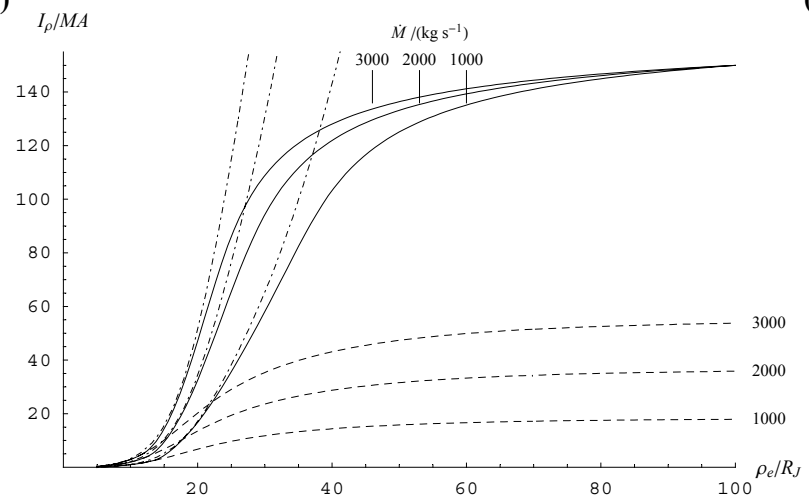

(b)

(c)
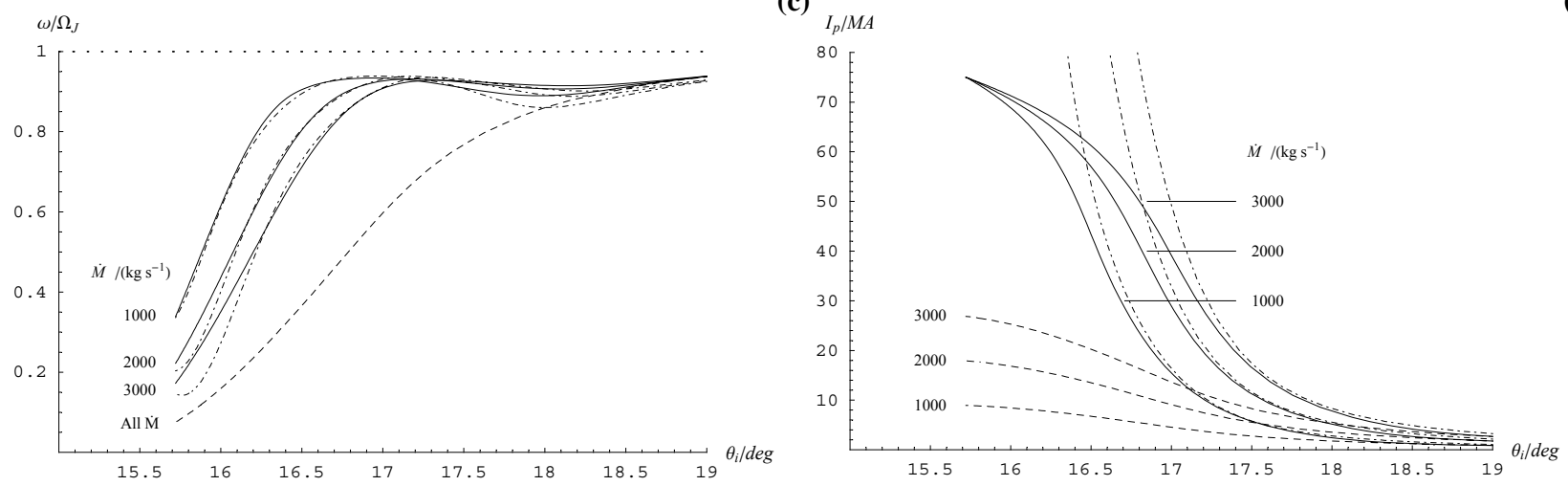

(d)

Fig. 17. Plots of the magnetosphere-ionosphere coupling current system parameters obtained using the empirical conductivity model (Eq. 37) (solid lines), constant conductivity $\Sigma_{P}^{*}=\Sigma_{P}^{*}\left(j_{\| i}=0\right)$ (dashed lines), and the near-rigid corotation approximations given by Eqs. (24)-(26) (dot-dashed lines), for three values of the iogenic plasma mass outflow rate $\dot{M}=1000,2000$ and $3000 \mathrm{~kg} \mathrm{~s}^{-1}$ (as indicated). The empirical conductivity model solutions have the boundary condition on $j_{\| i}$ set such that the value of the azimuth-integrated equatorial radial current in each case is equal to $150 \mathrm{MA}$. The solutions are tracked numerically to $12 R_{J}$, inside which they are completed by the "first-iteration" approximation of Sect. 4.2. The parameters in panels (a) and (b) are plotted versus jovicentric equatorial radial distance $\rho_{e}$, while those in panels (c)-(h) are plotted versus dipole co-latitude $\theta_{i}$. Plot (a) shows the plasma angular velocity $\omega$ normalised to the planet's angular velocity $\Omega_{J}$, plot (b) shows the azimuth-integrated equatorial radial current $I_{\rho}$ in MA, plot (c) shows the plasma angular velocity normalised to the planet's angular velocity $\Omega_{J}$, plot (d) shows the azimuth-integrated ionospheric Pedersen current $I_{P}$ in MA, plot (e) shows the fieldaligned current density at the feet of the field lines $j_{\| i}$ in $\mu \mathrm{A} \mathrm{m}^{-2}$, plot (f) shows the effective height-integrated Pedersen conductivity $\Sigma_{P}^{*}$ in mho, plot (g) shows the minimum accelerating voltage in $\mathrm{kV}$ required to drive the field-aligned current obtained from the exact form of Eq. (15), while plot (h) shows the precipitating energy flux $E_{f}$ in $\mathrm{mW} \mathrm{m}^{-2}$ obtained from the exact form of Eq. (16).

fluxes which produce the elevated conductivities are shown in Figs. 16e and 16f, respectively. The peak voltages are $\sim 50 \mathrm{kV}$, falling somewhat with increasing $\dot{M}$, with peak energy fluxes of $\sim 14 \mathrm{~mW} \mathrm{~m}^{-2}$, again falling somewhat with increasing $\dot{M}$, in a region whose width (FWHM) is $\sim 0.6^{\circ}$ $(\sim 800 \mathrm{~km})$, significantly narrower than the profiles for constant conductivity shown in Fig. 13e. These energy fluxes correspond to a UV luminosity of $\sim 80-140 \mathrm{kR}$ in this region, which compares reasonably with main oval observations (Clarke et al., 1998; Prangé et al., 1998; Grodent et al., 2003).

Finally, in order to display results which cover a reasonable range of variations within the Jovian magnetosphere, in Figs. 17 and 18 we show results in a similar format for the same conductivity model, but where the value of the radial current at the outer boundary at $100 R_{J}$ is fixed at values which are a factor of 1.5 larger (Fig. 17) and 1.5 smaller
(Fig. 18) than in Figs. 15 and 16, i.e. at $150 \mathrm{MA}$ and $67 \mathrm{MA}$, respectively. In order to keep the presentation reasonably compact, however, we simply show the plasma angular velocity and total equatorial radial current plotted versus $\rho_{e}$ in Figs. 17a, b and Figs. 18a, b, and the angular velocity, Pedersen current, field-aligned current, Pedersen conductivity, accelerating voltage and precipitating electron flux plotted versus co-latitude in Figs. 17c-h and Figs. 18c-h, respectively. Comparison with Figs. 15 and 16 show that when the field-perpendicular current is increased to $150 \mathrm{MA}$, the angular velocities are elevated and the field-aligned current is increased by a factor of $\sim 1.8$, while its spatial distribution is shifted polewards by $\sim 0.2^{\circ}$. Consequently, the accelerating voltages are increased to $\sim 100 \mathrm{kV}$ in this case, and the peak energy fluxes to $\sim 50 \mathrm{~mW} \mathrm{~m}^{-2}$, corresponding to an enhanced UV auroral luminosity of $500 \mathrm{kR}$. The latitudinal region in which the energy fluxes achieve such values is 


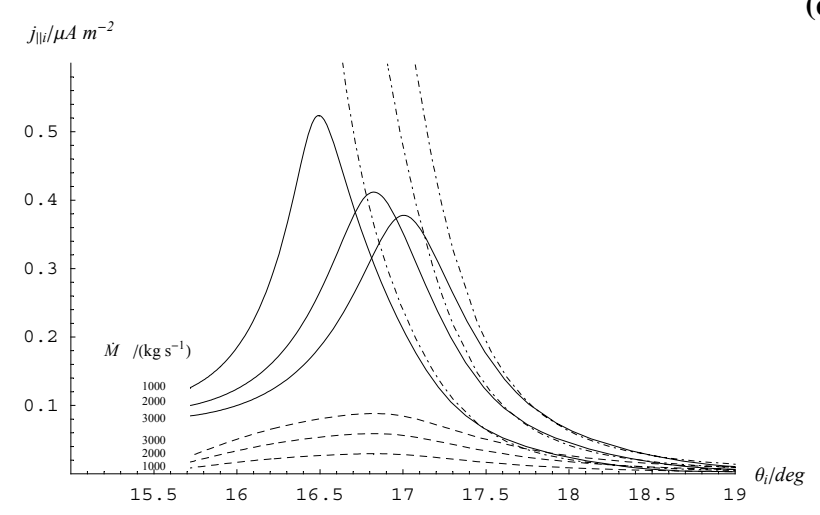

(e)

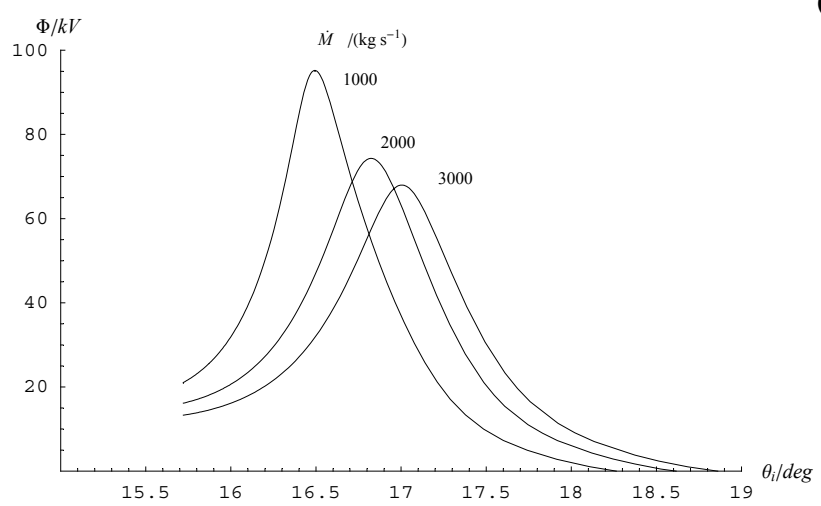

(g)

Fig. 17. Continued.

$\sim 0.5^{\circ}$ (FWHM), corresponding to $650 \mathrm{~km}$ in the ionosphere. By contrast, when the total current is reduced to $67 \mathrm{MA}$ at the boundary at $100 R_{J}$, the field-aligned currents are correspondingly reduced in rough proportion, as are the accelerating voltages to $\sim 25 \mathrm{kV}$, while the precipitating energy flux then falls to $\sim 5 \mathrm{~mW} \mathrm{~m}^{-2}$, corresponding to a weak UV luminosity of $50 \mathrm{kR}$. This extends over a latitudinal region $\sim 0.7^{\circ}$ (FWHM), corresponding to $910 \mathrm{~km}$.

\section{Summary and conclusions}

In this paper we have considered the problem of the coupling current system that flows between the ionosphere and the middle magnetosphere current sheet in the Jovian system, which imparts angular momentum to the plasma outflowing from the Io torus. In modelling this current system we have, for the first time, considered the enhancement in the ionospheric Pedersen conductivity which is produced by the precipitating energetic electrons in regions of upward-directed field-aligned current flow. The properties of the electron precipitation are estimated from Knight's (1973) kinetic theory, while the effects on the ionospheric conductivity have been determined using Millward et al.'s (2002) modelling results. For simplicity we have employed these inputs to derive a model for the dependence of the effective height-integrated Pedersen conductivity on the upward field-aligned current density, which is taken to be valid at all points in the mid-
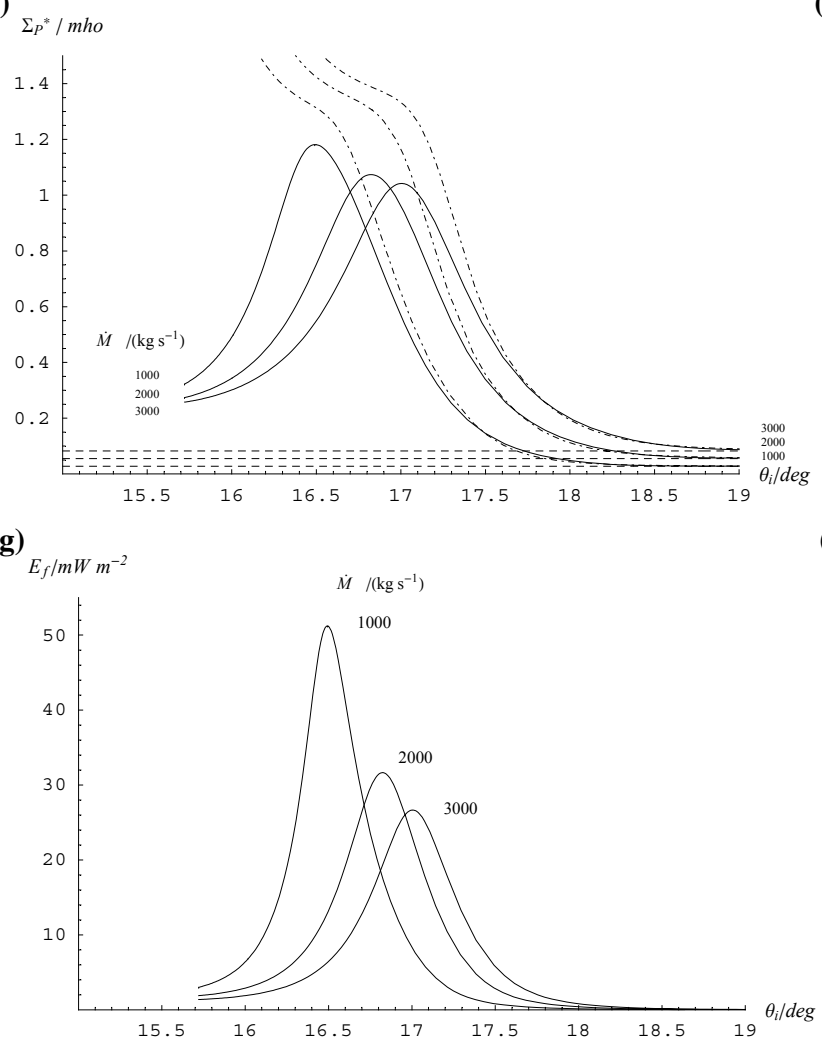

(h)

dle magnetosphere. Inclusion of the effects of spatial dependence would require development of models of the spatial dependence of the properties of the hot magnetospheric source electron population, that do not exist at the present time. We have also employed a number of other simplified models of the conductivity dependence on the field-aligned current, which have allowed us to examine how the results depend on the properties of the model. We have then incorporated these models into the description of the M-I system, solving for the currents with the changing conductivity selfconsistently included. One element of inconsistency which remains, however, is that the Knight (1973) theory requires the presence of field-aligned voltages of $\sim 20-100 \mathrm{kV}$ on the auroral field lines, whose effects are not included in our mapping of the flow between the magnetosphere and ionosphere, where we have assumed equipotential field lines. This factor should be taken into account in future work, but is not a priori expected to lead to very major effects, since the voltages concerned are very small compared with the total voltages across the middle magnetosphere current sheet of the order of $\sim 10 \mathrm{MV}$.

Following definition of the Pedersen conductivity model as discussed above, two coupled first-order differential equations must then be solved simultaneously, the first being the Hill-Pontius equation for the plasma angular velocity based on Newton's laws, the second being the current continuity equation (Eqs. 12 and 14 above). To define a particular solution then requires the choice of two boundary conditions, one 


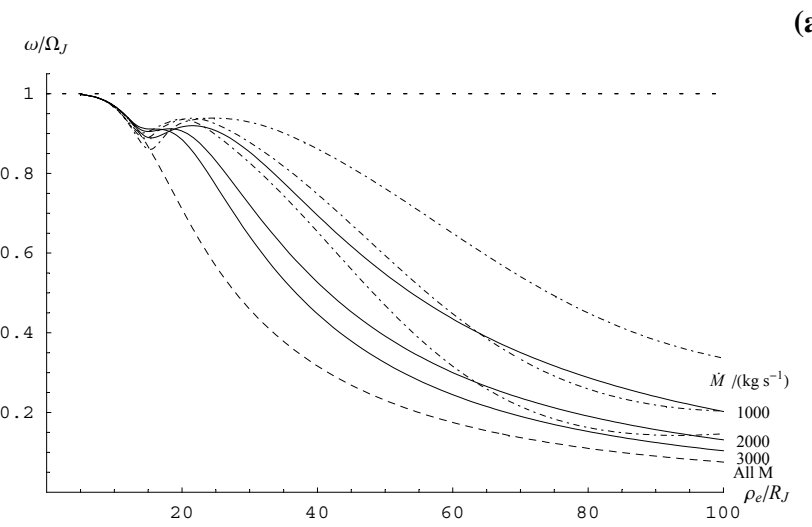

(a)

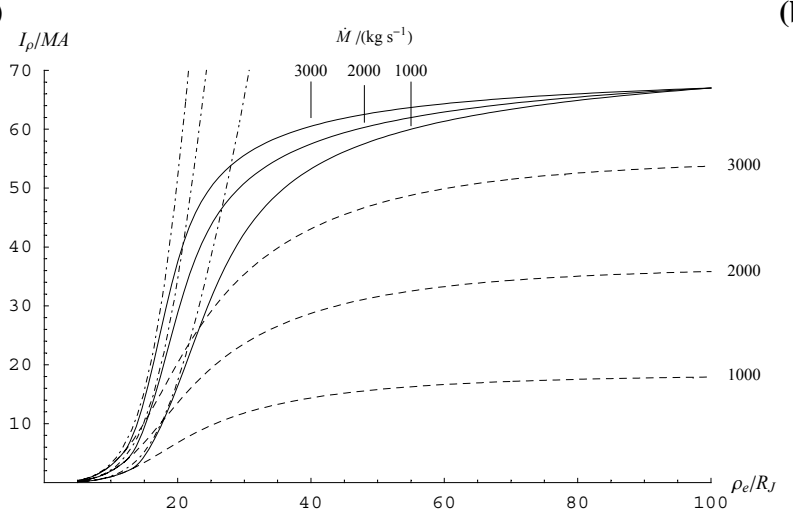

(c)
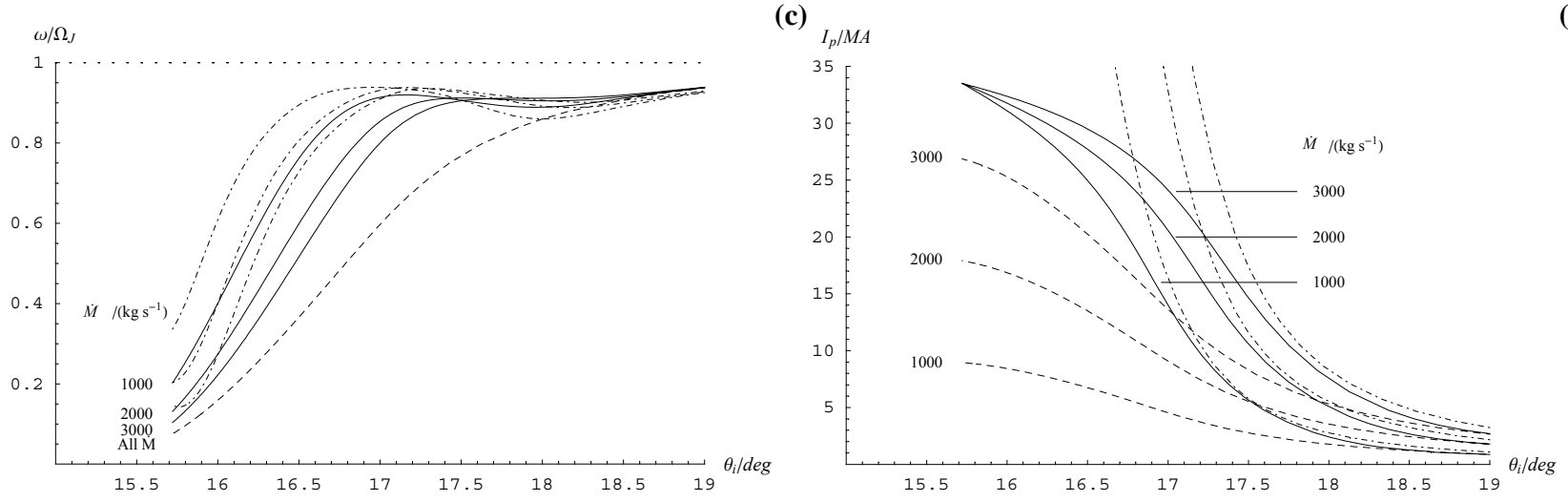

(e)
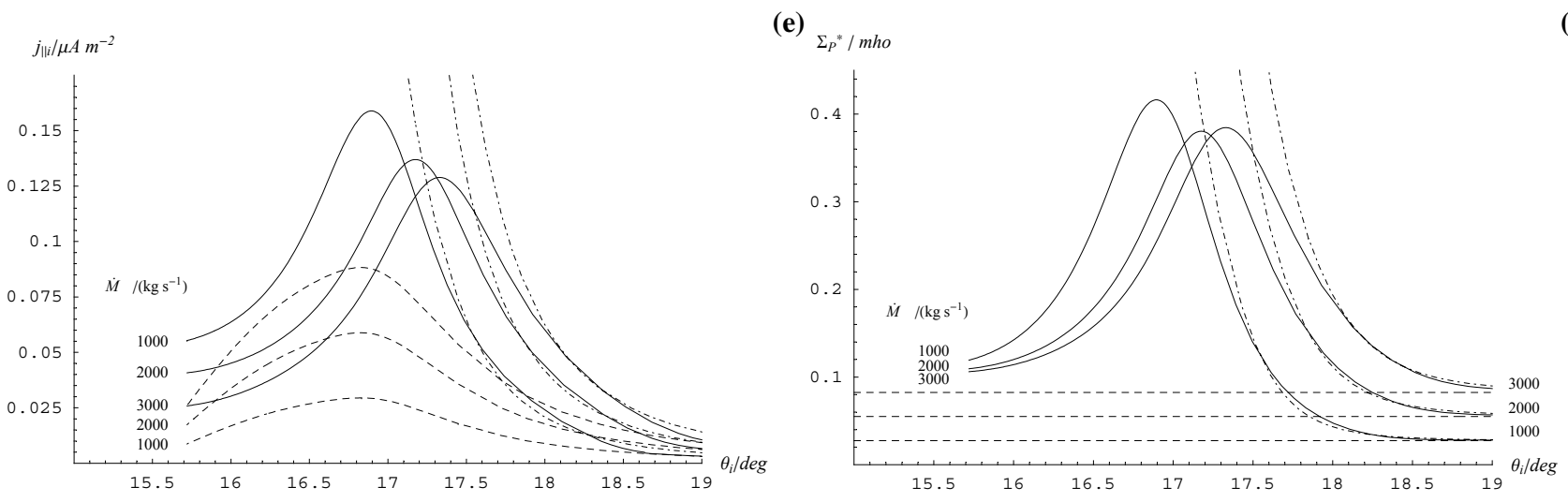

(g)
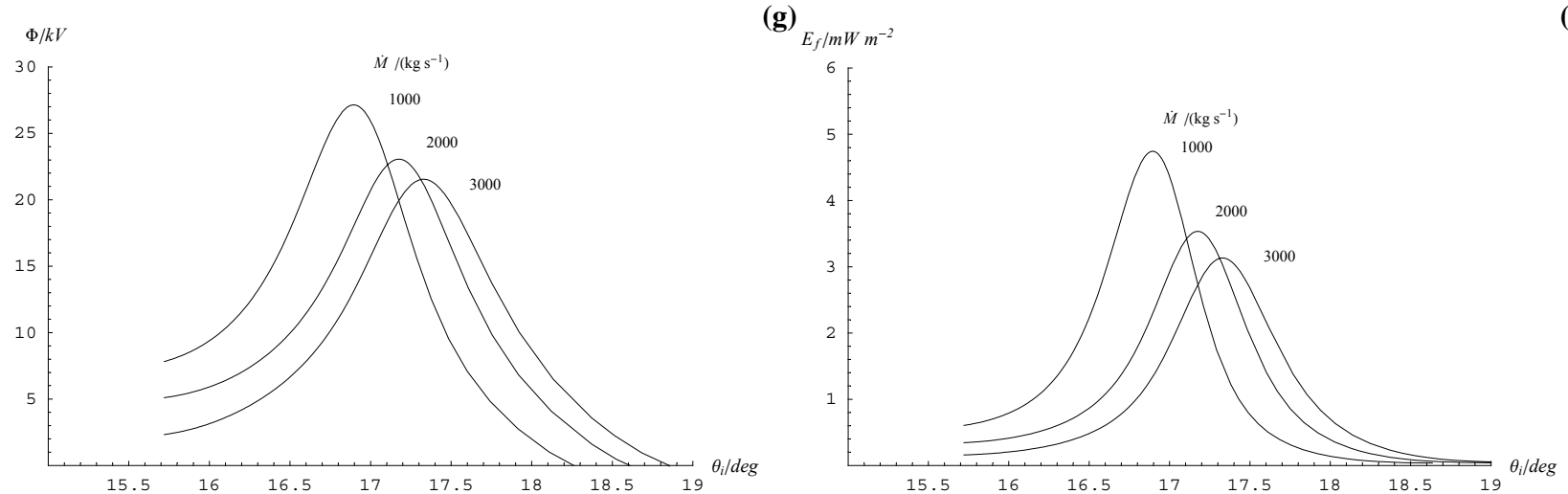

(h) (b)

(d)

Fig. 18. As Fig. 17 except with the boundary condition $I_{\rho}\left(100 R_{J}\right)=67 \mathrm{MA}$. 
of which is set by the requirement that the plasma angular velocity does not diverge at small radial distances, but rather that the plasma rigidly corotates with the planet in this limit. Practically, however, the solution of the equations requires the choice of values of the plasma angular velocity and fieldaligned current at one boundary location, taken here to be the outer boundary of the model current sheet at $100 R_{J}$, with the choice of the field-aligned current also being equivalent to the choice of the ionospheric conductivity at the outer boundary, via the conductivity model employed. In the majority of the calculations we have then held the field-aligned current (and therefore also the ionospheric conductivity) constant at the boundary and have then iterated the angular velocity to find the solution that does not diverge at small distances.

The solutions so obtained show a number of important features which are different from those obtained previously with constant assumed ionospheric conductivities, and which potentially resolve some outstanding issues concerning the distribution of plasma angular velocity and current in Jupiter's middle magnetosphere. First, concerning the plasma angular velocity, because the field-aligned current density falls to small values in the innermost regions, the precipitationinduced enhancement of the ionospheric conductivity is also small in this region. The angular velocity thus tends to fall relatively rapidly with distance in the innermost region, out to $\sim 15 R_{J}$ in the models derived in Sect. 5 , which were intended to represent reasonably realistic conditions in the Jovian magnetosphere. Thus, for example, Hill (1980) derived values of the Pedersen conductivity of $\sim 0.05$ mho for typical iogenic source rates of $\sim 2000 \mathrm{~kg} \mathrm{~s}^{-1}$ from Voyager-1 angular velocity data in the inner region. However, the field-aligned current and conductivity grow rapidly on field lines that map to the equator at distances beyond $\sim 15 R_{J}$ (corresponding to dipole co-latitudes poleward of $\sim 18^{\circ}$ in the ionosphere), thus maintaining the plasma angular velocity at much higher values in the outer region than would be obtained from the conductivities deduced in the inner region. This result thus confirms the conjecture of Cowley and Bunce (2001) to this effect, and provides an explanation of the elevated angular velocities deduced from energetic ion anisotropies in the outer region by Kane et al. (1995) and by Krupp et al. (2001). In our "realistic" models we then find that the field-aligned current peaks at values $\sim 0.22-0.28 \mu \mathrm{A} \mathrm{m}^{-2}$ on field lines which map in the equatorial plane to distances of $\sim 20-30 R_{J}\left(16.7^{\circ}-17.3^{\circ}\right.$ co-latitude in the ionosphere), with the peak field-aligned currents corresponding to peak ionospheric Pedersen conductivities of $\sim 0.7 \mathrm{mho}$, according to our model. The fieldaligned currents and conductivities then fall to smaller values at larger distances, determined by the choice of boundary condition. In our "realistic" models this has been set by imposing the condition that the total radial current flowing in the equatorial current sheet reaches $100 \mathrm{MA}$ at $100 R_{J}$, in conformity with the results derived from Galileo azimuthal magnetic field data by Khurana (2001). The resulting solutions for the current system are then found to be very similar in form to that derived from the magnetospheric field data, with the upward-directed field-aligned current input into the current sheet being concentrated in the inner part of the system at $\sim 25 R_{J}$ in the equatorial plane, such that the equatorial radial current becomes plateaued at near-constant values at distances beyond. However, matching the position of the field-aligned current input deduced from Galileo data, centred at $\sim 20 R_{J}$, favours large values of the iogenic source rate, of around $3000 \mathrm{~kg} \mathrm{~s}^{-1}$ or more. Such large values seem rather unrealistic when compared with a range of previous estimates of the plasma production rate within the torus, and the (smaller) outward transport rate within the equatorial plasma disc. These suggest instead an upper limit on the outward plasma transport rate of $\sim 2000 \mathrm{~kg} \mathrm{~s}^{-1}$, with $\sim 1000 \mathrm{~kg} \mathrm{~s}^{-1}$ being a more typical value. A possible explanation of this discrepancy which is suggested by Eq. (25) (which should be valid in the region in question), is that the strength of the north-south field threading through the current sheet in this region is too large in existing models. This possibility should be examined in future work.

We finally note that the location of the upward-directed field-aligned currents deduced in this model are comparable with the observed location of the Jovian main auroral oval deduced from HST and Galileo data (e.g. Prangé et al., 1998; Clarke et al., 1998; Vasavada et al., 1999; Grodent et al., 2003). Although the field-aligned current is directed out of the ionosphere into the equatorial current sheet over the whole of the current sheet, as found in previous calculations (such that the current must close outside of the middle magnetosphere in the poleward region not described by the model), the concentration of the field-aligned current in the inner region, relative to solutions with constant conductivity, leads to a related concentration in the ionosphere. We then find in our realistic models that the peaks in the field-aligned current map to $16.7^{\circ}-17.3^{\circ}$ co-latitude in the ionosphere for mass outflow rates of $1000-3000 \mathrm{~kg} \mathrm{~s}^{-1}$, respectively, in a region whose FWHM is $\sim 1^{\circ}$ (1200 km north - south). The corresponding accelerating field-aligned voltages required by Knight's (1973) theory are then $\sim 50 \mathrm{kV}$, and the peak precipitating electron energy fluxes are $\sim 14 \mathrm{~mW} \mathrm{~m}^{-2}$, located in a region of FWHM $\sim 0.6^{\circ}(\sim 800 \mathrm{~km}$ north - south $)$, the latter energy flux then resulting in a main oval UV aurora of $\sim 140 \mathrm{kR}$.

Acknowledgements. JDN was supported during the course of this study by a PPARC Quota Studentship, and SWHC by PPARC Senior Fellowship PPA/N/S/2000/00197.

Topical Editor T. Pulkkinen thanks a referee for his help in evaluating this paper.

\section{References}

Achilleos, N., Miller, S., Tennyson, J., Aylward, A. D., MuellerWodarg, I., and Rees, D.: JIM: A time-dependent, threedimensional model of Jupiter's thermosphere and ionosphere, J. Geophys. Res., 103, 20 089, 1998.

Bagenal, F.: Empirical model of the Io plasma torus, J. Geophys. Res., 99, $11043,1994$.

Bagenal, F.: The ionization source near Io from Galileo wake data, Geophys. Res. Lett., 24, 2111, 1997. 
Belcher, J. W.: The low-energy plasma in the jovian magnetosphere, in: Physics of the Jovian Magnetosphere, edited by Dessler, A. J., Cambridge Univ. Press, Cambridge, UK, 68, 1983.

Broadfoot, A. L., Sandel, B. R., Shemansky, D. E., McConnell, J. C., Smith, G. R., Holberg, J. B., Atreya, S. K., Donahue, T. M., Strobel, D. F., and Bertaux, J. L.: Overview of the Voyager ultraviolet spectrometry results through Jupiter encounter, J. Geophys. Res., 86, 8259, 1981.

Brown, M. E.: Observations of mass loading in the Io torus, Geophys. Res. Lett., 21, 10, 847, 1994.

Bunce, E. J. and Cowley S. W. H.: Divergence of the equatorial current in the dawn sector of Jupiter's magnetosphere: analysis of Pioneer and Voyager magnetic field data, Planet. Space Sci., 49, 1089, 2001.

Clarke, J. T., Ballester, G., Trauger, J., Ajello, J., Pryor, W., Tobiska, K., Connerney, J. E. P., Gladstone, G. R., Waite Jr., J. H., Ben Jaffel, L., and Gérard, J.C.: Hubble Space Telescope imaging of Jupiter's UV aurora during the Galileo orbiter mission, J. Geophys. Res., 103, 20 217, 1998.

Connerney, J. E. P., Acuña, M. H., and Ness, N. F.: Modeling the Jovian current sheet and inner magnetosphere, J. Geophys. Res., 86, 8370, 1981

Connerney, J. E. P., Acuña, M. H., Ness, N. F., and Satoh, T.: New models of Jupiter's magnetic field constrained by the Io flux tube footprint, J. Geophys. Res., 103, 11 929, 1998.

Cowley, S. W. H. and Bunce E. J.: Origin of the main auroral oval in Jupiter's coupled magnetosphere-ionosphere system, Planet. Space Sci., 49, 1067, 2001.

Cowley, S. W. H. and Bunce, E. J.: Modulation of jovian middle magnetosphere currents and auroral precipitation by solar windinduced compressions and expansions of the magnetosphere: Initial conditions and steady state, Planet. Space Sci., 51, 31, 2003.

Cowley, S. W. H., Nichols, J. D., and Bunce E. J.: Steady-state distributions of flow, current, and auroral precipitation in Jupiter's middle magnetosphere: Solutions for current sheet and dipole magnetic field models, Planet. Space Sci., 50, 717, 2002.

Cowley, S. W. H., Bunce, E. J., and Nichols, J. D.: Origins of Jupiter's main oval auroral emissions, J. Geophys. Res., 108 (A4), 8002, doi:10.1029/2002JA009329, 2003.

Delamere, P. A. and Bagenal, F.: Modeling variability of plasma conditions in the Io torus, J. Geophys. Res., 108 (A7), 1276, doi:10.1029/2002JA009706, 2003.

Edwards, T. M., Bunce, E. J., and Cowley, S. W. H.: A note on the vector potential of Connerney et al.'s model of the equatorial current sheet in Jupiter's magnetosphere, Planet. Space Sci., 49, 1115, 2001.

Grodent, D., Clarke, J. T., Kim, J., Waite Jr., J. H., and Cowley, S. W. H.: Jupiter's main oval observed with HST-STIS, J. Geophys. Res., 108, (A11), 1389, doi:10.1029/2003JA009921, 2003.

Hill, T. W.: Inertial limit on corotation, J. Geophys. Res., 84, 6554, 1979.

Hill, T. W.: Corotation lag in Jupiter's magnetosphere: Comparison of observation and theory, Science, 207, 301, 1980.

Hill, T. W.: The jovian auroral oval, J. Geophys. Res., 106, 8101, 2001

Hill, T. W., Dessler, A. J., and Goertz, C. K.: Magnetospheric models, in: Physics of the Jovian Magnetosphere, edited by Dessler, A. J., Cambridge Univ. Press, Cambridge, UK, 353, 1983.

Huang, T. S. and Hill, T. W.: Corotation lag of the jovian atmosphere, ionosphere and magnetosphere, J. Geophys. Res., 94, 3761, 1989.
Kane, M., Mauk, B. H., Keath, E. P., and Krimigis, S. M.: Hot ions in the jovian magnetodisc: A model for Voyager 2 low-energy charged particle measurements, J. Geophys. Res., 100, 19473, 1995.

Khurana, K. K.: Influence of solar wind on Jupiter's magnetosphere deduced from currents in the equatorial plane, J. Geophys. Res., 106, 25 999, 2001.

Khurana, K. K. and Kivelson, M. G.: Inference of the angular velocity of plasma in the jovian magnetosphere from the sweepback of magnetic field, J. Geophys. Res., 98, 67, 1993.

Knight, S.: Parallel electric fields, Planet. Space Sci., 21, 741, 1973.

Krupp, N., Lagg, A., Livi, S., Wilken, B., Woch, J., Roelof, E. C., and Williams, D. J.: Global flows of energetic ions in Jupiter's equatorial plane: First-order approximation, J. Geophys. Res., 106, $26017,2001$.

Lundin, R. and Sandahl, I.: Some characteristics of the parallel electric field acceleration of electrons over discrete auroral arcs as observed from two rocket flights, Symposium on European Rocket Research, ESA SP-135, 125, 1978.

Millward, G., Miller, S., Stallard, T., Aylward, A. D., and Achilleos, N.: On the dynamics of the jovian ionosphere and thermosphere III, The modelling of auroral conductivity, Icarus, 160, 95, 2002.

Nichols, J. D. and Cowley, S. W. H.: Magnetosphere-ionosphere coupling currents in Jupiter's middle magnetosphere: dependence on the effective ionospheric Pedersen conductivity and iogenic plasma mass outflow rate, Ann. Geophys., 21, 1419, 2003.

Pallier, L. and Prangé, R.: More about the structure of the high latitude jovian aurorae, Planet. Space Sci., 49, 1159, 2001.

Paschmann, G., Haaland, S., and Treumann, R.: Auroral plasma physics, edited by Paschmann, G., Haaland, S., and Treumann, R., Kluwer Publ., Dordrecht, 2002.

Pontius Jr., D. H.: Radial mass transport and rotational dynamics, J. Geophys. Res., 102, 7137, 1997.

Pontius Jr., D. H. and Hill, T. W.: Departure from corotation of the Io plasma torus: Local plasma production, Geophys. Res. Lett., 9, 12, 1321, 1982.

Prangé, R., Rego, D., Pallier, L., Connerney, J. E. P., Zarka, P., and Queinnec, J.: Detailed study of FUV jovian auroral features with the post-COSTAR HST faint object camera, J. Geophys. Res., 103, 20 195, 1998.

Satoh, T., Connerney, J. E. P., and Baron, R. L.: Emission source model of Jupiter's $\mathrm{H}_{3}^{+}$aurorae: A generalized inverse analysis of images, Icarus, 122, 1, 1996.

Scudder, J. D., Sittler Jr., E. C., Bridge, H. S.: A survey of the plasma electron environment of Jupiter: a view from Voyager, J. Geophys. Res., 86, 8157, 1981.

Siscoe, G. L. and Summers, D.: Centrifugally-driven diffusion of iogenic plasma, J. Geophys. Res., 86, 8471, 1981.

Southwood, D. J. and Kivelson, M. G.: A new perspective concerning the influence of the solar wind on Jupiter, J. Geophys. Res., 106, 6123, 2001.

Strobel, D. F. and Atreya, S. K.: Ionosphere, in: Physics of the Jovian Magnetosphere, edited by Dessler, A. J., Cambridge Univ. Press, Cambridge, UK, 51, 1983.

Vasavada, A. R., Bouchez, A. H., Ingersoll, A. P., Little, B., and Anger, C. D.: Jupiter's visible aurora and Io footprint, J. Geophys. Res., 104, 27 133, 1999.

Vasyliunas, V. M.: Plasma distribution and flow, in Physics of the Jovian Magnetosphere, edited by Dessler, A. J., Cambridge Univ. Press, Cambridge, UK, 395, 1983. 